\title{
ANÁLISE DE EXPERIMENTOS EM BLOCOS CASUALIZADOS COMPLETOS AUMENTADOS (BLOCOS DE FEDERER), COM PARCELAS SUBDIVIDIDAS NO TEMPO
}

\author{
EDIO VIZONI \\ (Matematico)
}

Orientador: Prof, Dr. ROBERTO SIMIONATO MORAES

Dissertação apresentada à Escola Superior de Agricultura "Luiz de Queiroz", da Universidade de São Paulo, para obtenção do título de Mestre em Agronomia. Área de Concentração em Estatística $\theta$ Experimentação Agronômica.

\footnotetext{
$P \mid R A C I C A B A$

Estado de São Paulo - Brasil

Outụbro - 1984
} 
. $i$.

A meu pai ( in memorian)

ofereso.

Dedico

A minha mãe

Aos meus fizhos

As minhas irmãs. 


\section{AGRADECIMENTOS}

Ao Dr. Roberto Simionato Moraes, pela orientação se gura e pelo incentivo para a execução deste trabalho.

Ao Dr. Humberto de Campos, Coordenador do Curso de Pós-Graduação em Estatśtica e Experimentação Agronômica, pelas sugestões e ensinamentos.

Aos Drs. Isaias Rangel Nogueira, Antonio. Francisco Iemma, Cássio Roberto de Melo Godoi, Maria Cristina Stolf Nogueira, bem como todos os professores do curso de Pós-Graduação em Estatistica e Experimentação Agronômica da ESALQ/USP, por sua aco lhida, seus ensinamentos e solicitude com a qual invariantemente, nos atenderam dentro e fora das salas de aula.

Aos colegas do Departamento de Matemática aplicada, da "FUEL", Departamento de Matemātica da "FAFICLA", e da UNIDADE POLO, pelo apoio e amizade.

$\bar{A}$ FUNDAÇÃO UNIVERSIDADE ESTADUAL DE LONDRINA(FUEL), pelo afastamento concedido.

A FACULDADE DE FILOSOFIA CIENCIAS E LETRAS DE ARAPONGAS ( FAFICLA), pelo afastamento concedido. 
A CAPES pela bolsa de estudos concedida.

A SEC - Secretaria de Educação e Cultura do Paraná pela licença concedida.

A Srta. Rosa Maria Alves e ao Dr. Cássio Roberto de Melo Godoi pela versão do Resumo para o Inglês.

Aos companheiros do Curso de Pós-Graduação, pela receptividade, amizade e estímulo.

A Maria Luiza Meneghetti, e seus familiares, pelo apoio, compreensão, incentivo e amizade.

A Maria das Graças de Lima, pela execução dos trabalhos de datilografia.

A minha família, pela paciência e dedicação.

A todos que direta ou indiretamente contribuiram na execução deste trabalho. 


\section{IND ICE}

Pág.

RESUMO

$v i i i$

SUMMARY

$x i$

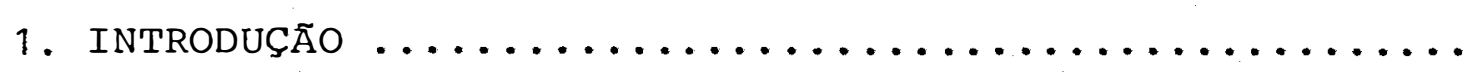

2. REVISÃO BIBLIOGRĂFICA $\ldots \ldots \ldots \ldots \ldots \ldots \ldots \ldots \ldots \ldots$

3. MATERIAL

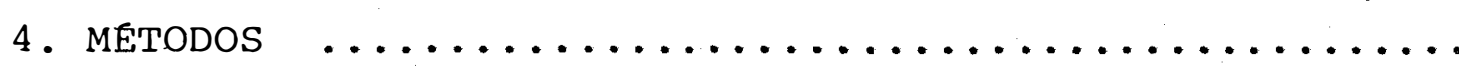

4.1 . Introdução $\ldots \ldots \ldots \ldots \ldots \ldots \ldots \ldots \ldots \ldots$

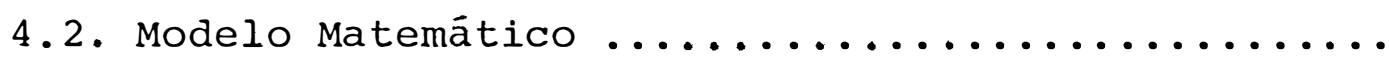

4.3. Equações Normais .....................

4.4. Solução do Sistema de Equações ..............

4.5. Análise de Variância ...................

4.5 .1 . Introdução ....................

4.5.2. Soma de Quadrados dos Parâmetros .........

4.6. Esperança das Somas de Quadrados ............. 4.6.1. Média Geral .....................

4.6.2. Tratamentos Principais ..............

4.6 .3$. Blocos $\ldots \ldots \ldots \ldots \ldots \ldots \ldots \ldots \ldots \ldots \ldots \ldots \ldots \ldots \ldots \ldots \ldots$ 
4.6.4. Soma de Quadrados de Parcel-as ........... 57

4.6 .5 . Resíduo (a) ...................... 60

4.6.6. Tratamentos Secundários .............. 61

4.6.7. Interação txa $\ldots \ldots \ldots \ldots \ldots \ldots \ldots \ldots \ldots . \ldots \ldots$

4.6.8. Interação bxa .................. 80

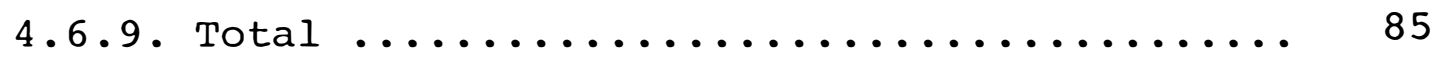

4.6 .10 . Residuo (b) $\ldots \ldots \ldots \ldots \ldots \ldots \ldots \ldots \ldots$

4.6.11. Quadro de Análise de Variância .......... 88

4.7. Dispersão para Tratamentos ............... 89

4.7.1. Tratamentos Principais ............. 89

4.7.2. Tratamentos Secundários .............. 95

4.7.3. Dispersão para a Interação txa ......... 97

4.8. Ajuste de Médias ...................... 97

4.9. Comparações Múltiplas ................. 98

4.9.1. Para os Tratamentos Principais ......... 99

4.9.2. Para os Tratamentos Secundários .......... 100

4.10. Testes Sobre a Estrutura da Matriz de Covariâncias ............................ 101

4.10.1. Teste de Homogeneidade para as matrizes Covariâncias ...................... 101

4.10.2. Teste de Uniformidade da matriz comum de Covariâncias ..................... 103

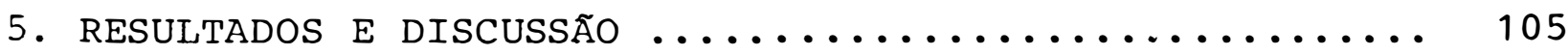

5.1. Teste de Homogeneidade e Uniformidade de matriz de Covariâncias $\ldots \ldots \ldots \ldots \ldots \ldots \ldots \ldots \ldots \ldots \ldots \ldots \ldots \ldots \ldots \ldots \ldots$ 
5.2. Equações Normais ......................... 108

5.3. Quadro de Análise de Variância .............. 109

5.4. Comparações Mültiplas ..................... 111

6. CONCLUSOES ................................... 113

7. REFERENCIAS BIBLIOGRAFICAS ...................... 114

8. APENDICE ................................... 120

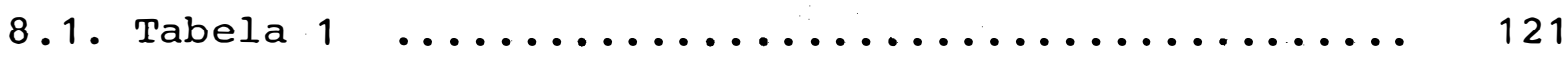

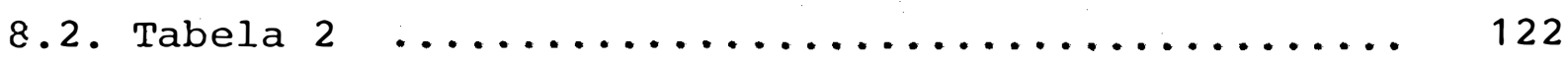


TITULO: -ANÁLISE DE EXPERIMENTOS EM BLOCOS CASUALIZADOS COMPLE TOS AUMENTAdOS (BLOCOS DE FEDERER), COM PARCELAS SUBDIVIDIDAS NO TEMPO.

AUTOR: EDIO VIZONI.

ORIENTADOR: Dr. ROBERTO SIMIONATO MORAES.

\author{
R E S U M O
}

No teste de novos tratamentos (variedades, metodologias, produtos químicos, etc) nem sempre é possível se obter quantidades suficientes desses novos produtos, para um número razoāvel de repetições.

FEDERER (1956), desenvolveu uma metodologia para testes de clones de cana-de-açúcar, a qual permite realizar a anālise de variância e as comparações múltiplas entre os efeitos de tratamentos, tanto entre as variedades comerciáveis já existentes (comuns), ou ainda entre as novas variedades ou clones (regulares), bem como a comparação entre as comuns e regulares, baseando-se em delineamentos inteiramente casuali zados, blocos, ou quadrados latinos, aos quais deu o nome de "delineamentos aumentados". 
Neste trabalho, pretendeu-se fazer um estudo do de lineamento em blocos aumentados com parcelas subdivididas no tempo, tendo por finalidade, principalmente a aplicação em ex perimentos agronômicos, com culturas perenes, aproveitando as produções de anos sucessivos.

Considerou-se, c, variedades comuns dispostas em b blocos, e $\mathrm{Z}$ variedades regulares as quais aparecem uma única vez em todo o experimento e $\mathrm{v}$ anos - E, ainda que todos os blocos tenham o mesmo número de parcelas $(K)$, nada impedindo entretanto que esses números sejam diferentes.

Adotou-se o seguinte modelo matemätico:

$y_{\ell j v}=m+t_{\ell}+b_{j}+(t b)_{\ell j}+a_{v}+t a_{\ell v}+b a_{j v}+e_{\ell j v}$

Quando se trabalha com parcelas subdivididas

no tempo, não é raro encontrar correlações diferentes entre duas subparcelas de uma mesma parcela. Neste estudo, porser um tra balho inicial, considerou-se apenas o caso onde estas correlações são constantes e existe a independēncia entre subparce las de parcelas distintas, resultando: 


$$
\operatorname{cov}\left(y_{l j v^{\prime}} y_{\left.l^{\prime} j^{\prime} v^{\prime}\right)}=\left\{\begin{array}{l}
\sigma^{2} \text { se } l=\ell^{\prime} ; j=j^{\prime} ; v=u^{\prime} \\
\rho \sigma^{2} \text { se } \ell=\ell^{\prime} ; j=j^{\prime} ; v \neq v^{\prime} \\
0 \text { em outros casos }
\end{array}\right.\right.
$$

Nestas condições, baseando-se na metodologia apresen tada por PIMENTEL GOMES (1967), para blocos incompletos balan ceados, e no trabalho desenvolvido por IEMMA (1981), sobre parcelas subdivididas, determinou-se: o sistema de equações nor mais, somas de quadrados dos parâmetros, as matrizes de disper são, os critērios para as comparações múltiplas pelo teste de Tukey . 
ANALYSIS OF EXPERIMENTS IN COMPLETELY RANDOMISED AUGMENTED BLOCKS DESIGN (FEDERER BLOCKS), WITH SPLIT PLOT ON TIME

Author: EDIO VIZONI

Adviser: ROBERTO SIMIONATO MORAES

\section{$S$ U M M A R Y}

In the new treatments test (varieties, methodologies, chemical products) it is not possible to obtain sufficient quantities of these new products, for a rasonable num ber of repetitions.

FEDERER (1956), developed a methodology for tests in sugar cane clones, that allows one do the analysis of va riance and multiple comparisons among the treatments effects, either between commercial existent varieties (common), or bet ween the new varieties or clones (regular), either the comparisons between the common and regular varieties, based on a completely randomised, blocks, or lattice square designs, na mely the augmented designs. 
In this dissertation, we intended to study the augmented block design with split plot on time, objecting mainly, the aplication in agronomic experiments, with perennial cultures, utilizing production of successive years.

We considered c commons varieties in b blocks, and $z$ regular varieties that appear only once in all experiments in $\underline{v}$ years. Although every block have the same num ber of plots (K), we allow different number of plots.

We adopted the following mathematical model:

$$
y_{1 j v}=m+t_{1}+b_{j}+(t b)_{1 j}+a_{v}+t a_{1 v}+b a_{j v}+e_{1 j v}
$$

When we work with split plot in time, it is not unusual to find different correlations between two sub plot in the same plot. As a preliminary study we considered only the case where this correlations are constant. And also have the independency between subplots of different plots, resulting:

$$
\operatorname{cov}\left(y_{1 j v} y_{I^{\prime}} j^{\prime} v^{\prime}\right)=\left\{\begin{array}{cc}
\sigma^{2} & \text { if } 1=1^{\prime} ; j=j^{\prime} ; v=v^{\prime} \\
0 \sigma^{2} & \text { if } 1=1^{\prime} j=j^{\prime} ; v^{\prime} v^{\prime} \\
0 & \text { in the others cases. }
\end{array}\right.
$$


With thos conditions, based in the methodology developed by PIMENTEL GOMES (1967), for balanced incomplete blocks, an in the paper developed by IEMMA (1981), about split plot, it was determined: the normal equations system, the parameter sum of squares, the dispersion matrices, the multiple comparison criterion by Tukey test. 


\section{INTRODUÇÃO}

Quando se pretende comparar novas variedades, tra tamentos, ou novas metodologias, com outras já existentes nem sempre é possivel obter quantidades suficientes desses materiais para um número de repetições, que possa ter uma eficiên cia razoável para a análise de variância.

FEDERER (1956), introduziu o chamado "MODELO AUMENTADO", num programa de seleção de novos clones de cana-deaçúcar do "HAWAIIAN SUGAR PLANTER'S ASSOCIATION", em substituição a modelos anteriores. Este podendo ser realizado em de lineamento inteiramente ao acaso, blocos casualizados, quádrados latinos, etc. 
Estes modelos apresentam dois grupos de tratamen tos:

a) C - Tratamentos comuns, repetidos b vezes.

b) Z - Tratamentos regulares, aparecendo uma única vez em todo o experimento.

FEDERER (1961.a, 1961.b), FEDERER, et alli(1975), desenvolveram uma metodologia onde os tratamentos regulares (clones ou variedades), podem ou não ser repetidos o mesmo nú mero de vezes.

Como nem sempre é possível obter quantidades suficientes desses materiais, para se realizar mais de uma parcela, pensou-se em aproveitar as produções ou observações sucessivas de uma mesma parcela como repetição dessa variedade. Diante dessa nova idéia, pensou-se em utilizar o delineamento em blocos completos com parcelas subdivididas no tempo, tendo-se como tratamentos principais as variedades, e para tratamentos secundários as produções nos vários anos sucessivos.

Sabemos que quando se trabalha com parcelas subdivididas no tempo, não raramente encontramos correlações diferentes entre duas subparcelas de uma mesma parcela.

Neste estudo inicial, considerou-se somente o cạ so onde essas correlações são constantes. E existe a independência entre subparcelas de parcelas distintas, resultando: 
$\operatorname{cov}\left(y_{\ell j v^{\prime}} y_{\left.\ell^{\prime} j^{\prime} v^{\prime}\right)}=\left\{\begin{array}{l}\sigma^{2}, \text { se } \ell=\ell^{\prime} ; j=j^{\prime} ; v=v^{\prime} \\ \rho \sigma^{2}, \text { se } \ell=\ell^{\prime} ; j=j^{\prime} ; v \neq v^{\prime} \\ 0 \text { em outros casos. }\end{array}\right.\right.$

considerando assim, somente o caso em que as matrizes de varí âncias e covariâncias, por parcelas, são homogêneas e uniformes.

Considerou-se, ainda, apenas o caso onde as varie dades comuns são repetidas b vezes, e as variedades regulares aparecem uma única vez em todo o experimento, e o nūmero de parcelas e subparcelas por blocos sejam iguais, com a finalidade unicamente de simplificar o tratamento algébrico. 


\section{REVISĀO BIBLIOGRÁFICA}

Devido a necessidade de um delineamento experimental mais eficiente na comparação de plântulas, ("seedlings") e variedades ("clones"), nas fases iniciais de um programa de melhoramento de cana-de-açúcar, abacaxi, etc. FEDERER (1956) desenvolveu uma metodologia que permite comparar estas novas variedades, chamadas de variedades regulares, com variedades já existentes, ou comerciáveis, chamadas de comuns; chamando este novo delineamento de "Delineamento aumentado", que é um tipo de delineamento experimental, formado a partir dos delineamentos inteiramente ao acaso, blocos, linhas e colunas. .

Estes delineamentos apresentam dois grupos de tratamentos, os comuns, repetidos b vezes, e um segundo grupo, os regulares, que aparecem uma única vez no experimento. 
Comparando os trabalhos de FEDERER (1956) e PIMENTEL GOMES \& GUIMARÃES (1958), propondo uma análise intrablo cos de um grupo de experimento em blocos casualizados completos, onde alguns tratamentos são comuns para todos os grupos, observa-se que há uma certa semelhança entre elas, onde cada bloco do delineamento aumentado corresponde a um experimento em blocos casualizados completos, considerado sem repetição.

Novas sugestões foram apresentadas por: FEDERER (1961a, 1961b), PAVATE (1961), CORSTEN (1962), AFONJA (1969), PIMENTEL GOMES (1970), MARTINEZ (1972), dentre outros.

o grande uso dos experimentos em parcelas subdivididas, justificado pela sua simplicidade e alta eficiência, segundo LEONARD e CLARK (1939), foi proposto por Yates, em 1933, seguido por diversos autores como STELL e TORRIE (1960), SNEDECOR e COCHRAN (1967), PIMEṄTEL GOMES (1976), COCHRAN e COX (1976), dentre outros.

IEMMA (1981), desenvolvendo uma metodologia para ensaios com parcelas subdivididas em blocos incompletos, chama a atenção para o fato de que mesmo que os blocos sejam recomendados eles podem apresentar o inconveniente de fugir ao controle do experimentador, principalmente quando o nümero de tratamentos secundários for relativamente grande, mas deve ser preferido sobre o inteiramente ao acaso, se o efeito de blo- 
cos for significativo, apesar do risco da crescente perda de eficiência diretamente associada ao tamanho dos blocos, citan do COCHRAN e COX (1976), os quais definem os ensaios em parcelas subdivididas através dos ensaios em fatorial com confun dimento de efeitos principais, ressaltam sua eficiência em ex perimentos de campo, quando se deseja introduzir um fator adi cional dentro do ensaio.

Quando se trabalha com culturas perenes, onde se pode ter várias observações de uma mesma parcela, DINIZ (1980), considera duas situações básicas que pode ocorrer:

1ạ Cada unidade experimental é tratada somente uma vez durante o experimento ou recebe o mesmo tratamento repetidamente.

2a Cada unidade experimental recebe uma sequéência de tratamen tos sobre periodos sucessivos de tempo durante o curso do experimento.

Sendo que na 1ạ situação, estão incluídos experi mentos que estudam curvas de crescimento ou respostas e estudos de perfil; na 2 ă, os modelos do tipo change-over.

Classifica as observações de uma mesma parcela de duas formas:

a) Observações que diferem entre si pelas suas caracteristicas; 
b) Observações que tem a mesma característica, mas que diferem entre si somente quanto ao tempo.

Para as observações "a", o autor aconselha efetuar uma análise multivariada, ou uma análise univariada para cada característica observada; para as de "b", as naturezas dos dados são univariadas, usar uma análise univariada.

Para experimentos do tipo 1, com observações da parcela (b), dois tipos de análise univariada são, em geral, efetuadas,

1) Análise dos dados em cada tempo, separadamente.

2) Análise dos dados segundo o delineamento em parcelas subdi vididas no tempo.

Considerando-se as medidas repetidas sobre a mes ma parcela como um vetor de respostas, é válido um processo multivariado denominado análise de perfil.

Considerando-se ainda que é de se esperar que me didas adjacentes no tempo são mais altamente correlacionadas do que medidas distantes, esses dados, apesar de originados de um experimento aparentemente univariado, são essencialmente multivariados.

Mas quando se consideram as medidas repetidas so bre a mesma parcela como subparcelas no tempo, o delineamento em parcelas subdivididas, no tempo, pode ser empregado. No 
entanto, para a aplicação desse tipo de análise, o autor mostra as pressuposições que devem ser observadas.

19 Os tratamentos atribuidos às parcelas devem ser casualizados.

29 Os tratamentos atribuidos às subparcelas devem ser casuali zados (o que pode invalidar a análise, já que as observações repetidas no tempo não são casualizadas);

30 As medidas repetidas, tomadas sobre a mesma parcela, tem a mesma variância $\left(\sigma^{2}\right)$, e são igualmente correlacionadas, ou seja, que medidas adjacentes no tempo são igualmenté corre lacionadas com as medidas distantes.

Assim, a matriz de variâncias e covariâncias para todas as parcelas dos tratamentos comuns devem ser do tipo:

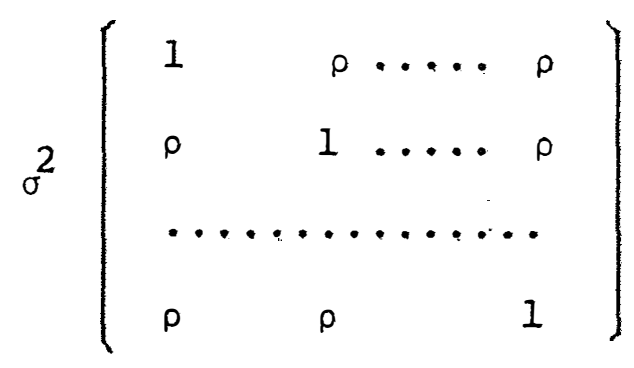

denominada "uniforme" por GEISSER (1963). 
Sugere também uma outra alternativa que algumas vezes possibilita o uso do modelo em parcelas subdivididas, que consiste em se trabalhar com as diferenças entre medidas sucessivas, ao invés de se usar os dados originais. o uso desta técnica algumas vezes reduz as variâncias e covariâncias, des tes efeitos para um grau suficiente que possibilite ser ignorada as correlações entre períodos sucessivos, já que as correlações entre períodos de tempo nem sempre são constantes; quando se usa este método, tempo e interação tratamento x tem po não podem ser testadas de maneira usual.

PARKER (1942), trabalhando com laranjeiras, veri ficou que as produções anuais não são igualmente correlaciona das, e devido a esse fato, SNEDECOR (1956) sugere uma análise baseada na soma das produções das parcelas e ANDERSON e BANCROFT (1952), sugerem que se separe o erro (b) em dois com ponentes BxA, BxTXA.

BOX (1950), considerando observações sucessivas na mesma parcela, acha válida uma análise simples, se puder supor que as observações tem as mesmas variâncias e mesmas co variâncias para todas as parcelas; e para verificar a validade da aplicação de uma análise de variância de um modelo em parcelas subdivididas, deve-se usar o procedimento proposto por BOX (1949), que recomenda verificar a hipótese de que a matriz de covariância seja a mesma, para todos os tratamentos; 
além disso, deve-se verificar se a matriz de variâncias e covariâncias é da forma:

$$
\Sigma=\left(\begin{array}{cccc}
a & b & \ldots & b \\
b & a & \ldots & b \\
\ldots & \ldots & \ldots & b \\
b & b & \ldots & a
\end{array}\right)
$$

designada "uniforme" por GEISSER (1963). Estabelece ainda um teste de significância aproximado para verificar a hipọtese de igualdade de variâncias e covariâncias.

No entanto, quando não se puder fazer tal suposi ção, ou seja, se as condições exigidas pelo modelo de parcelas subdivididas não forem satisfeitas, deve-se adotar uma ma triz de covariâncias de estrutura mais geral. Aconselha ainda, que em experimentos onde os resultados aparecem na forma de curvas de crescimento, a forma da matriz de covariância deve ser levada em consideração para se poder decidir sobre o mode 10 e o método de análise apropriados, pois, considera, ainda, que é de se esperar que a correlação entre medidas tomadas no tempo decresçam à medida que elas se distância, ou seja, se correlacionam serialmente.

Em situações onde a análise unịvariada não é apro priada, BOX (1950), propõe uma extensão multivariada de testes de significância, como procedimento alternativo. Para isso,u- 
tiliza o critério de wILKS (1932).

STEEL (1955), tomando as produções acumuladas pa ra cada ano em um ensaio de variedades de alfafa, compara as análises univariadas e multivariadas, mostrando que ambas apresentam os mesmos resultados.

STEEL e TORRIE (1960), lembram as diferenças existentes entre as análises de experimentos em parcelas subdi vididas no espaço e no tempo.

Salientam que em parcelas subdivididas no tempo pode acontecer grandes diferenças entre colheitas e entre suas variâncias. Outra diferença entre os dois tipos de experimentos é que os desvios padrões para várias comparações de médias de tratamentos não são sempre os mesmos em ambas as análises. Devido a isso, recomendam que seja feita uma análise de variância para cada colheita: Se a cultura apresentar mais de uma colheita por ano, sugerem a análise de cada colheita, separadamente, para então fazer-se a análise para cada ano, e dai a análise conjunta para anos.

Trabalhando com um experimento de variedades de alfafa, em blocos casualizados, dizem que a análise conjunta pode acontecer que a interação blocos x cortes seja relevante, devido a localização dos blocos. Devido a isso, a soma de qua drados da interação blocos $x$ cortes deve ser separada do erro (b) . 
PATTERSON e LOWE (1970), concordam que em ensaios de longa duração, existe a correlação serial entre as observa ções nas mesmas parcelas. Dizem ainda que se não for considerada essa correlação, pode-se perder eficiência na estimação dos efeitos de tratamentos e vícios na estimação do erro. No entanto, o vício na variância das médias de tratamentos pode ser eliminado completa ou parcialmente determinando-se dois erros: da parcela e parcela x erro. 
.13 .

\section{MATERIAL}

o material utilizado para a aplicação do método em estudo, refere-se a produção agrícola de cana-de-açúcar, da dos em toneladas por hectare, adapatados de um ensaio de sele ção de variedades.

O plantio das canas deu-se em Abril de 1970, ten do sido realizados 2 cortes, o 19 em Novembro de 1971, o 29 em Setembro de 1972 .

Utilizou-se no experimento 5 variedades comuns, dispostas em 5 blocos e 35 variedades regulares, as quais apa recem uma ünica vez em todo o experimento. Estes dados aparecem no apêndice, tabela 1 . 


\section{MÉTODOS}

\subsection{Introdução}

Para o desenvolvimento da metodologia, considerou-se um modelo de blocos completos casualizados aumentados (blocos de FEDERER), com $c$ tratamentos comuns e $z$ tratamentos regulares onde de cada parcela obteve-se $v$ observa̧̧ões relativos aos cortes em $v$ anos sucessivos, utilizandose assim o modelo de parcelas subdivididas, onde as $v$ obser vações no tempo correspondem as subparcelas.

$$
\text { Considera-se ainda que o número de parcelas em }
$$

todos os blocos fossem constantes e iguais a $K$, o nümero de tratamentos comuns repetidos $b$ vezes, e o nümero de tratamen tos regulares fossem iguais em todos os blocos e aparecendo em 
somente uma parcela em todo o experimento.

Em parcelas subdivididas, os tratamentos principais e os tratamentos secundários devem ser casualizados. Como as subparcelas neste caso, não podem ser casualizadas, segundo DINIZ (1980), poderá comprometer seriamente o uso do de-. lineamento, mostra então, que uma das exigências para a validade deste modelo'é em se fazer um teste de homogeneidade e uniformidade de matriz de variâncias e covariâncias.

4.2. Modelo Matemático

Considerou-se, para esse ensaio o modelo linear.

$$
y_{\ell j v}=m+t_{\ell}+b_{j}+(t b)_{\ell j}+a_{v}+(t a)_{\ell v}+(b a)_{j v}+e_{\ell j v}
$$

onde

$$
\begin{aligned}
l & =1,2, \ldots, c+z \\
c & =1 \ldots c \\
z & =1 \ldots z \\
j & =1 \ldots \ldots b \\
v & =1 \ldots \ldots
\end{aligned}
$$

para melhor visualịzação das matrizes, reescreveu-se o modelo (42.1) da seguinte forma

$$
y_{\ell j v}=m+\left(t_{i}+t_{i j}\right)+b_{j}+(t b)_{\ell j}+a_{v}+\left(t a_{i v}+t a_{i^{\prime} j v}\right)+b_{j v}+e_{\ell j v}
$$


onde

$y_{l j v}=e ́$ o valor observado na subparcela que recebeu o $v$-ésimo tratamento secundário dentro do $\ell$-êsimo tratamento prin cipal no j-ésimo bloco.

$m=$ é o efeito da média geral.

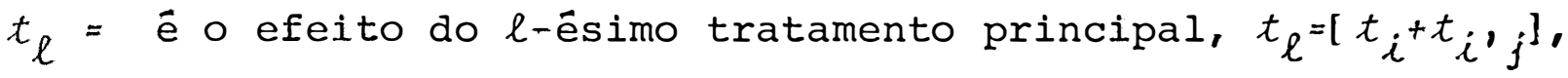
onde $t_{i}=$ efeito do $i$-ésimo tratamento principal comum $t_{i^{\prime} j}=$ efeito do $i^{\prime}$-ésimo tratamento principal regu lar do j-ésimo bloco.

$b_{j}=$ é o efeito do j-ésimo bloco.

$(t b)_{\ell j}=$ efeito da interação do $\ell$-ésimo tratamento principal com o j-ésimo bloco, também chamado de erro(a).

$a_{v}=$ efeito do v-ésimo tratamento secundārio (anos).

$|t a|_{\ell v}=$ é o efeito da interação entre o l-ésimo tratamento principal e o v-ésimo tratamento secundârio, sendo

$$
(t a)_{\ell v}=\left[t a_{i v}+t a_{i} j v\right] \quad \text { onde }
$$

$t a_{i v}=$ é o efeito da interação entre o i-ésimo tratamento principal comum e o v-ésimo tratamentose cundârio.

$t a_{i} j_{j}=$ é o efeito da interação entre o $i^{\prime}$-ésimo tratamento principal regular do j-ésimo bloco e o $v$ - ésimo tratamento secundário. 
$(b a)_{j v}=\hat{e}$ o efeito da interação entre o j-êsimo bloco e

$\circ$ v-ésimo tratamento secundârio.

$e_{\ell j v}=$ erro atribuído à observação $y_{\ell j v}$, chamado de erro (b).

Neste estudo consideroumse as seguintes restrições.

$$
\begin{aligned}
& b \sum_{i} z_{i}+\sum_{i^{\prime} j} \hat{t}_{i^{\prime} j}=0 \\
& \sum_{v} t \hat{a}_{i v}=0 \\
& \sum_{j} b_{j}=0 \\
& \sum_{v} t \hat{a}_{i}{ }_{j v}=0 \\
& \sum \bar{a}_{v}=0 \\
& b \sum_{i} t \hat{a}_{i v}+\sum_{i^{\prime} j} t \hat{a}_{i \prime j v}=0 \\
& \sum_{v} b \hat{a}_{j v}=0 \\
& \sum_{j} b \hat{a}_{j v}=0
\end{aligned}
$$

\subsection{Eçuações Normais}

Do modelo linear. $\mathrm{Y}=\mathrm{X} \theta+\varepsilon^{\prime}$ obtêm-se o sistema de equações normais

$$
X^{\prime} X \bar{\theta}=X^{\prime} Y
$$

onde $\mathrm{X}=\bar{e}$ a matriz dos coeficientes dos parâmetros de $\operatorname{dim}[n x(v+b+l+v \ell+v b)]$ sendo $n=b K v$ (número de sub-parcelas).

$\hat{\theta}=\bar{e}$ o vetor das soluções de mínimos quadrados, para os 
efeitos dos parâmetros de dimensão

$[(v+b+\ell+v \ell+v b) \times 1]$

$y=e ́$ o vetor de valores observados de dim $n \times 1$.

Fazendo-se a partição da matriz $\mathrm{X}$, e usando os mesmos conceitos sugeridos por IEMMA (1981), tem-se

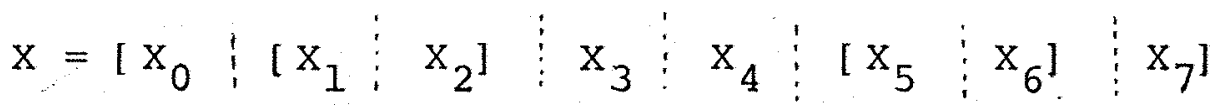

onde

$x_{0}=e ́$ o vetor que envolve a média geral dim $n \times 1$

$\mathrm{x}_{1}=$ é a matriz dos coeficientes associados aos tratamentos principais comuns dim $n \times c$.

$\mathrm{x}_{2}=$ é a matriz dos coeficientes associados aos tratamentos principais regulares, dim $n \times z$.

$\mathrm{X}_{3}=$ é a matriz dos coeficientes associados aos blocos, dim $n \times b$.

$\mathrm{x}_{4}=\mathrm{e}$ a matriz dos coeficientes associados aos tratamentos secundārios, dim $n \times v$.

$\mathrm{x}_{5}=$ è a matriz dos coeficientes associados as interações $t a_{i v}$, dim nxcv. 
$\mathrm{x}_{6}=$ é a matriz dos coeficientes associados as interaçöes ta ${ }_{i}^{\prime} j v$, dim $n x u z$.

$\mathrm{x}_{7}=$ é a matriz dos coeficientes associados as interações $b a_{j v}$, dim nxub.

Assim a matriz $X^{\prime} X$, resulta:

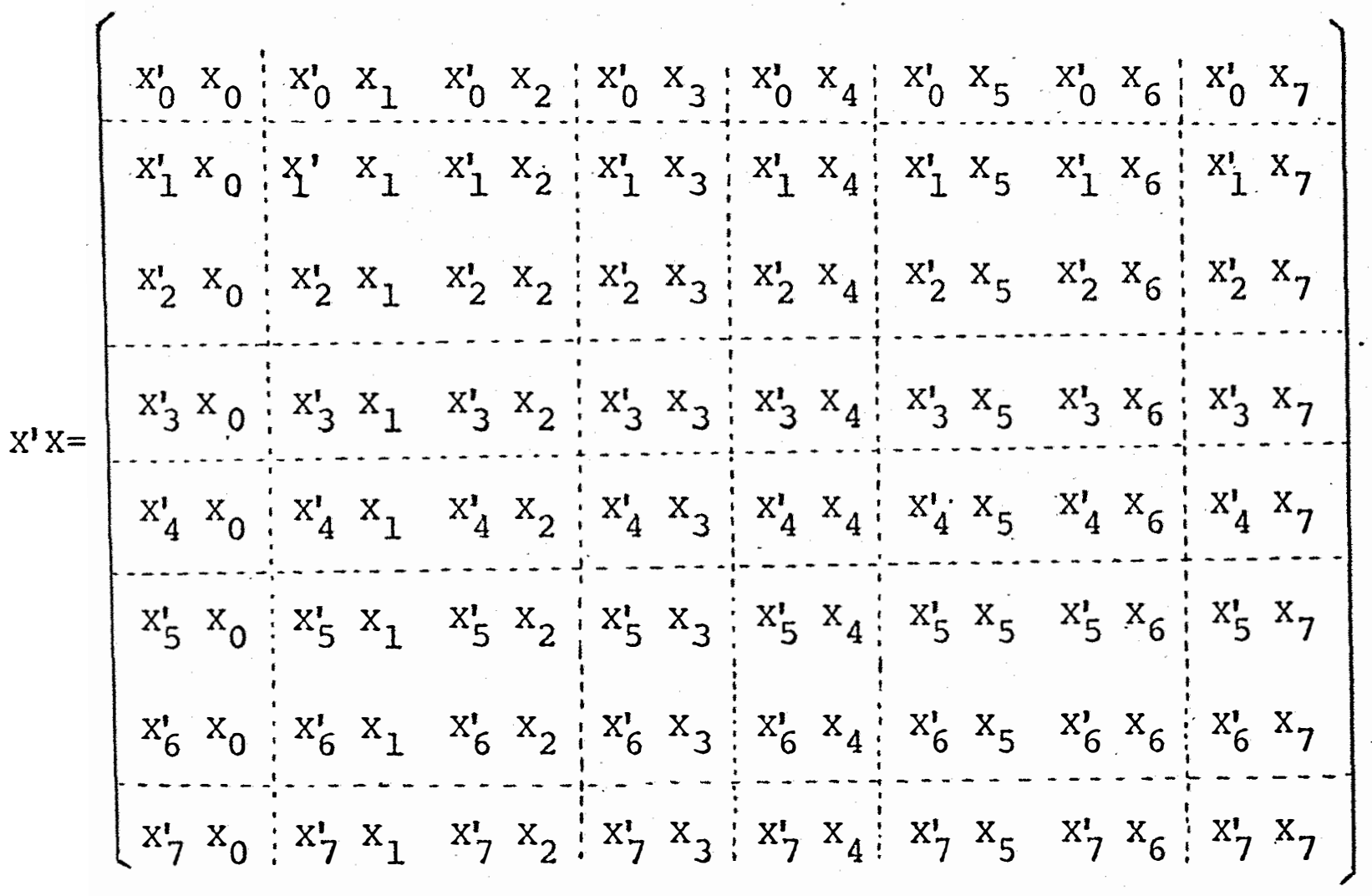

cujas partições chamaremos respectivamente de

$$
X^{\prime} X=\left(\begin{array}{cccccc}
n & g & c & d & e & f \\
g^{\prime} & R & N & P & S & D \\
C^{\prime} & N^{\prime} & A & K & V & F \\
d^{\prime} & P^{\prime} & K^{\prime} & U & H & G \\
\theta^{\prime} & S^{\prime} & V^{\prime} & H^{\prime} & I & B \\
F^{\prime} & D^{\prime} & F^{\prime} & G^{\prime} & B^{\prime} & W
\end{array}\right)
$$


onde

$\bar{n}=x_{0}^{\prime} x_{0}=b K v=$, no de subparcelas, dim $1 x I$

$g=\left[g_{1} ! g_{2}\right] ; g_{1}=x_{0}^{\prime} x_{1}=b v E_{(1 x c)}$, sendo $\mathbf{E}_{(1 \times c)}$, a matriz de $I^{\prime} s$

$$
g_{2}=x_{0}^{\prime} x_{2}=v E(1 \times z)
$$

$c=x_{0}^{\prime} x_{3}=K \cup \mathbf{E}(1 \times b)$

$a=x_{0}^{\prime} x_{4}=b K \mathbf{E}(1 \times v)$

$\mathrm{e}=\left[\begin{array}{l:l}\mathrm{e}_{1} & \mathrm{e}_{2}\end{array}\right] ; \quad \mathrm{e}_{1}=\mathrm{x}_{0}^{\prime} \mathrm{x}_{5}=b_{\mathrm{E}}(1 \times \mathrm{cv})$

$e_{2}=x_{0}^{\prime} x_{6}=E_{(1 x \cup z)}$

$f=x_{0}^{\prime} x_{7}=K \mathbf{E}_{(1 \times b v)}$

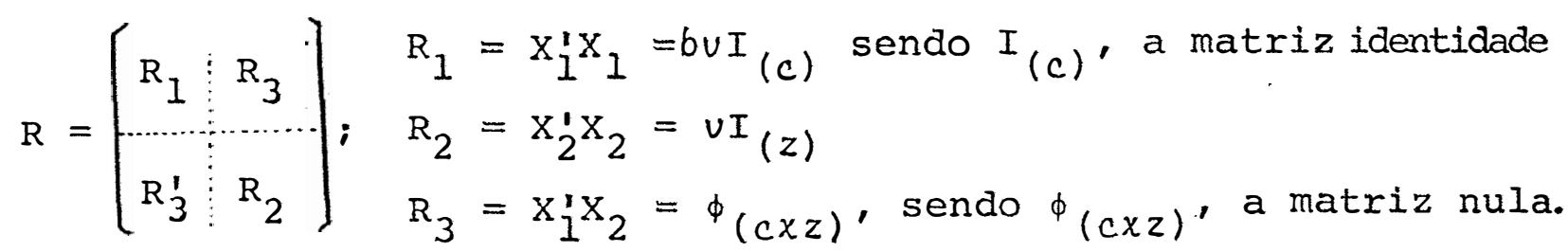

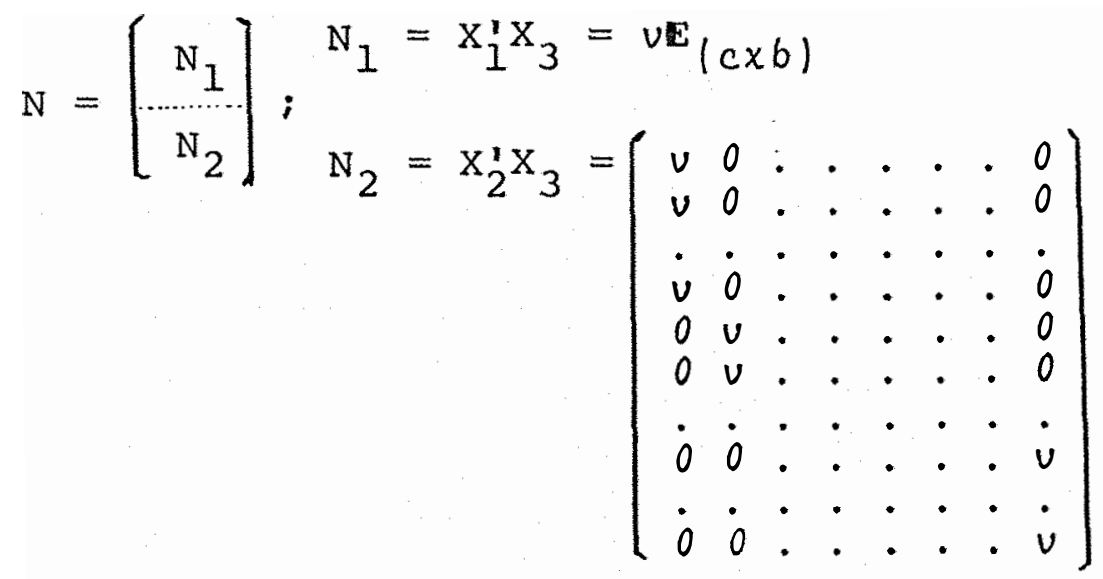

$(z \times b)$ 


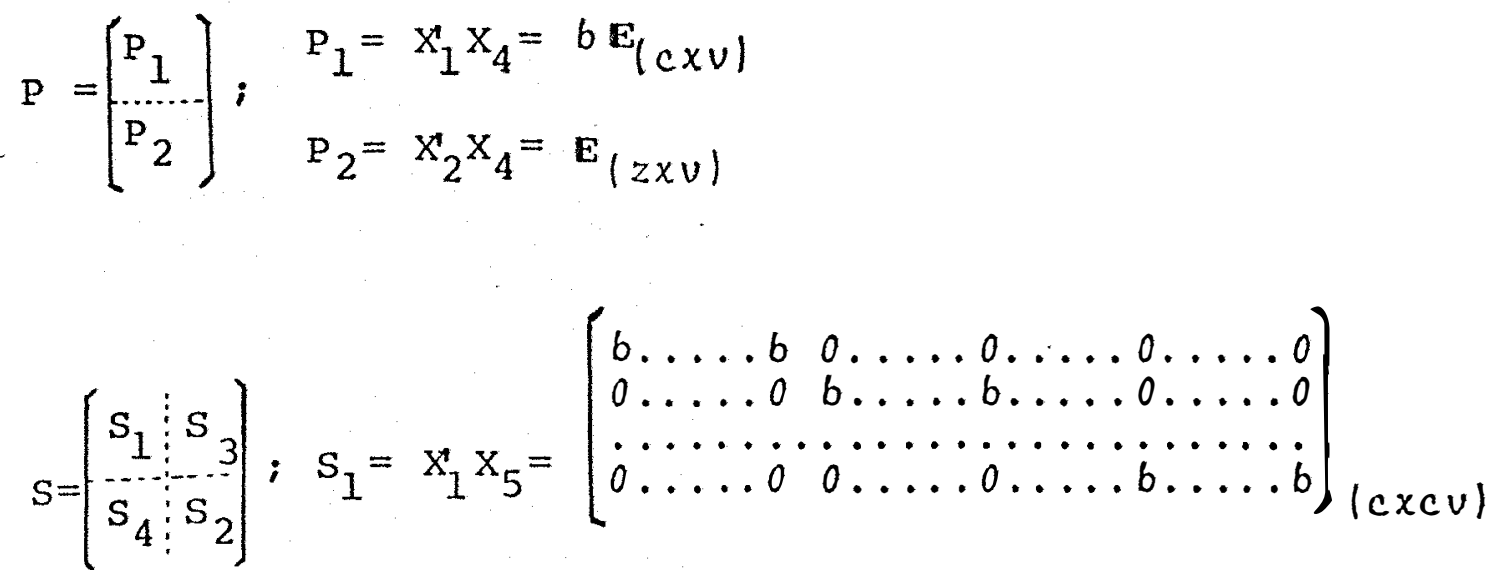

$$
\begin{aligned}
& S_{2}=x_{2} x_{6}=\left[I_{(z)} \ldots \ldots I_{(z)}\right] \text { (zxuz) } \\
& s_{3}=x_{1} x_{6}=\phi(c x v z) \\
& s_{4}=x_{2} x_{5}=\phi(z x c u)
\end{aligned}
$$

$D=\left(\begin{array}{c}D_{1} \\ \hdashline D_{2}\end{array}\right)$

$\mathrm{D}_{1}=\mathrm{x}_{1} \mathrm{x}_{7}=\mathrm{E}_{(\mathrm{c} \times b u)}$

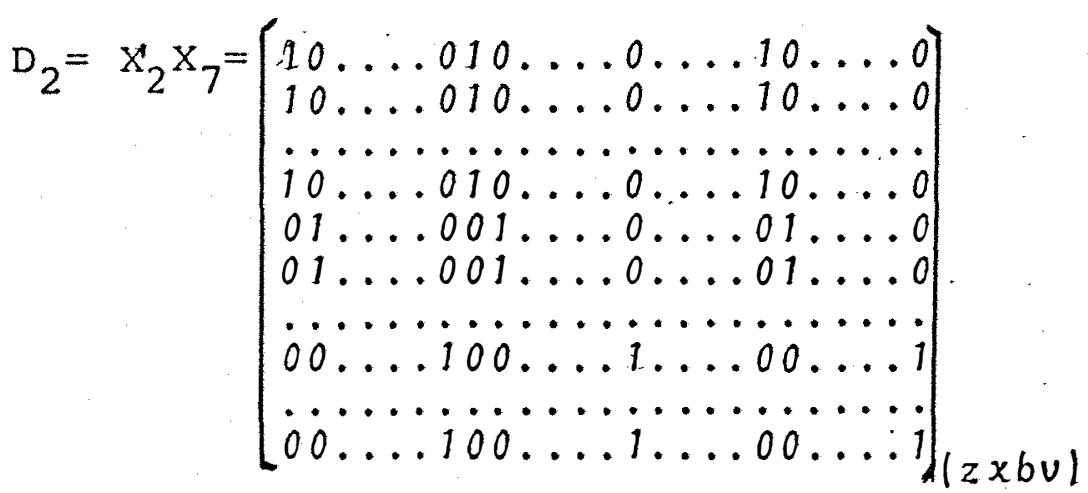

$$
\begin{aligned}
& A=X_{3} x_{3}=v K I(b) \\
& K=\mathrm{X}_{3} \mathrm{X}_{4}=K \mathbf{E}_{(b \times v)} \\
& \mathrm{V}=\left[\begin{array}{l:l}
\mathrm{V}_{1} & \mathrm{v}_{2}
\end{array}\right] ; \mathrm{v}_{1}=\mathrm{x}_{3} \mathrm{x}_{5}=\mathbf{E}_{(\mathrm{b} \times \mathrm{cu})}
\end{aligned}
$$

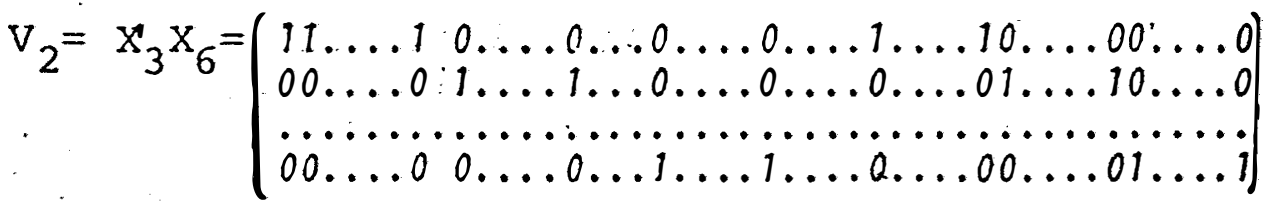




$$
\begin{aligned}
& F=X_{3} X_{7}=K\left[I_{(b)} \ldots I_{(b)}\right](b \times b u) \\
& U=X_{4}^{\prime} X_{4}=b K I(v)
\end{aligned}
$$

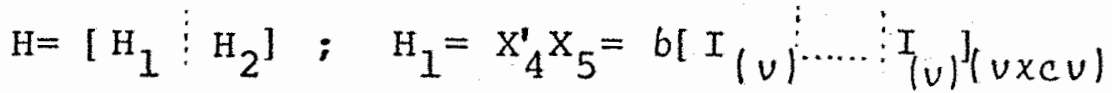

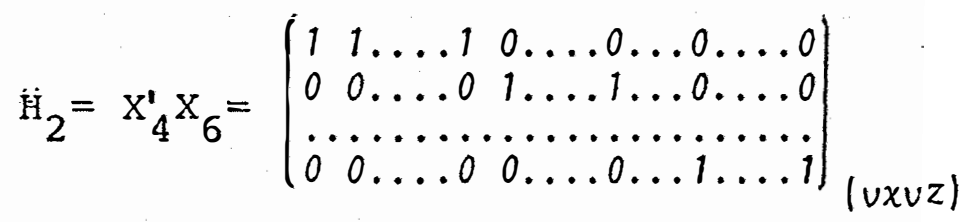

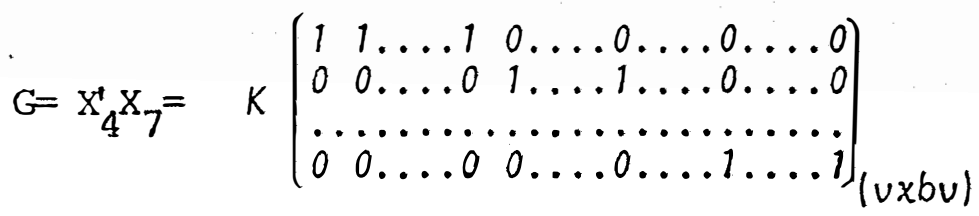

$$
\begin{aligned}
L=\left(\begin{array}{c:c}
L_{1} & I_{3} \\
\hdashline I_{3}^{\prime} & I_{2}
\end{array}\right) ; \quad L_{1}=X_{5}^{\prime} X_{5}=b I_{(c v)} \\
L_{6}^{\prime} X_{6}=I_{(v i)} \\
L_{3}=X_{5}^{\prime} X_{6}=\phi_{(c v x v z)}
\end{aligned}
$$

$B=\left[\begin{array}{c}B_{1} \\ -B_{2}\end{array}\right]$

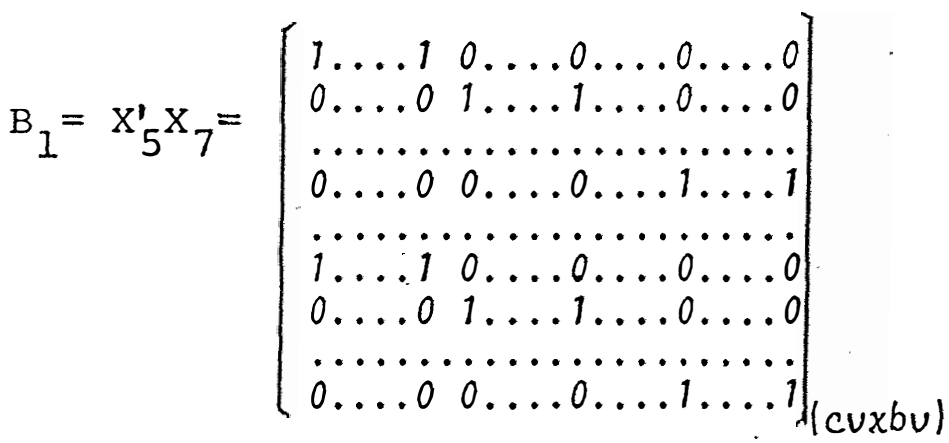




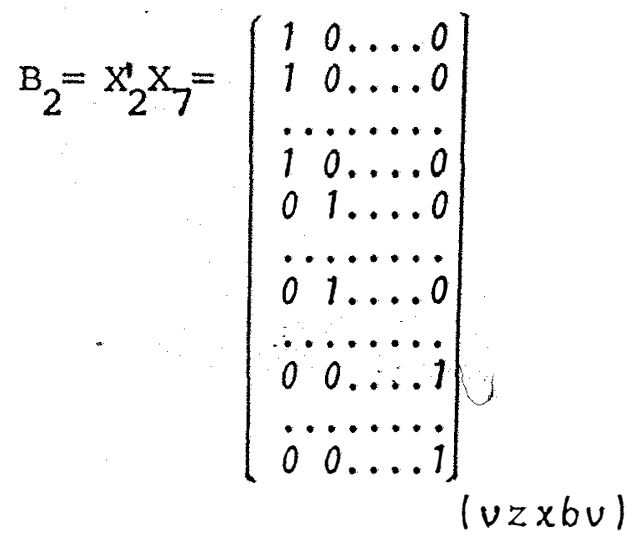

$W=X_{7}^{\prime} x_{7}=K I(b u)$

vetor de soluções

$\hat{\theta}$,

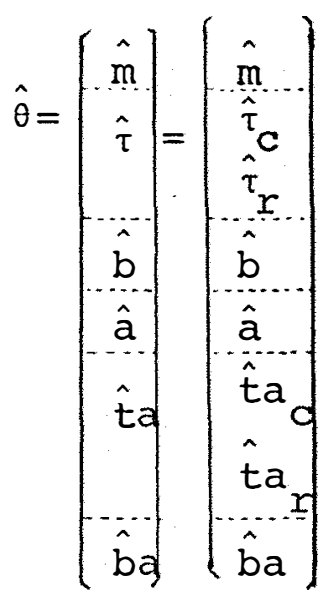

onde, considerando-se as restrições impostas em (4.2).

$\hat{\mathrm{m}}=$ é $\mathrm{o}$ efeito da média geral estimada, dim $1 \times 1$.

$\hat{\tau}_{C}=e$ o vetor dos efeitos estimados para tratamentos principais comuns.

$$
\hat{\tau}_{c}^{\prime}=\left[\hat{t}_{1} \hat{t}_{2} \ldots . \hat{t}_{c}\right](1 \times c)
$$

$\hat{\tau}_{r}=\bar{e}$ o vetor dos efeitos estimados para tratamentos principais regulares.

$$
\hat{\tau}_{r}^{\prime}=\left[\hat{t}_{11} \hat{t}_{21} \ldots \hat{t}_{k 1} \hat{t}_{12} \hat{t}_{22} \ldots \hat{t}_{k 2} \ldots \hat{t}_{1 b} \hat{t}_{2 b} \ldots \hat{t}_{k b}\right]_{(1 x z)}
$$


$\hat{b}=e ́$ o vetor dos efeitos estimados para os blocos,

$$
\hat{b}^{\prime}=\left[\hat{b}, \hat{b}_{2} \ldots \hat{b}_{b}\right]_{(1 \times b)}
$$

$\hat{a}=\bar{e}$ o vetor dos efeitos estimados para os tratamentos secundārios

$$
\hat{a}^{\prime}=\left[\hat{a}_{1} \hat{a}_{2} \ldots \hat{a}_{v}\right](1 \times v)
$$

$\hat{t}_{c}=\bar{e}$ o vetor dos efeitos estimados das interações tratamen tos comuns $\mathrm{x}$ tratamentos secundārios.

$$
\hat{t}_{c}^{\prime}=\left[\hat{t a}_{11} \hat{t a}_{12} \ldots \hat{t}_{1} \hat{t}_{12} \ldots \hat{t a}_{2 v} \ldots \hat{t}_{c 1} \hat{t a}_{c 2} \ldots \hat{t a}_{c v}\right]_{(1 x c v)}
$$

$\hat{t}_{r}=\bar{e}$ o vetor dos efeitos estimados das interações tratamen tos regulares $\mathrm{x}$ tratamentos secundários.

$$
\hat{t a}_{c}^{\prime}=\left[\hat{t} a_{111} \hat{t a}_{211} \ldots \hat{t a}_{k 11} \hat{t}_{121} \ldots \hat{t}_{k 21} \ldots \hat{t} a_{1 b u} \ldots \hat{t} a_{k b v}\right]_{(1 \times k b v)}
$$

$\hat{b} \mathrm{a}=$ é o vetor dos efeitos estimados das interações blocos $\mathrm{x}$ tratamentos secundārios.

$$
\hat{b} a^{\prime}=\left[\hat{b} a_{11} \hat{b} a_{21} \ldots \hat{b} a_{b 1} \hat{b} a_{12} \ldots \hat{b} a_{b 2} \ldots \hat{b} a_{1 v} \ldots \hat{b} a_{b v}\right]
$$


na matriz $X^{\prime} X$

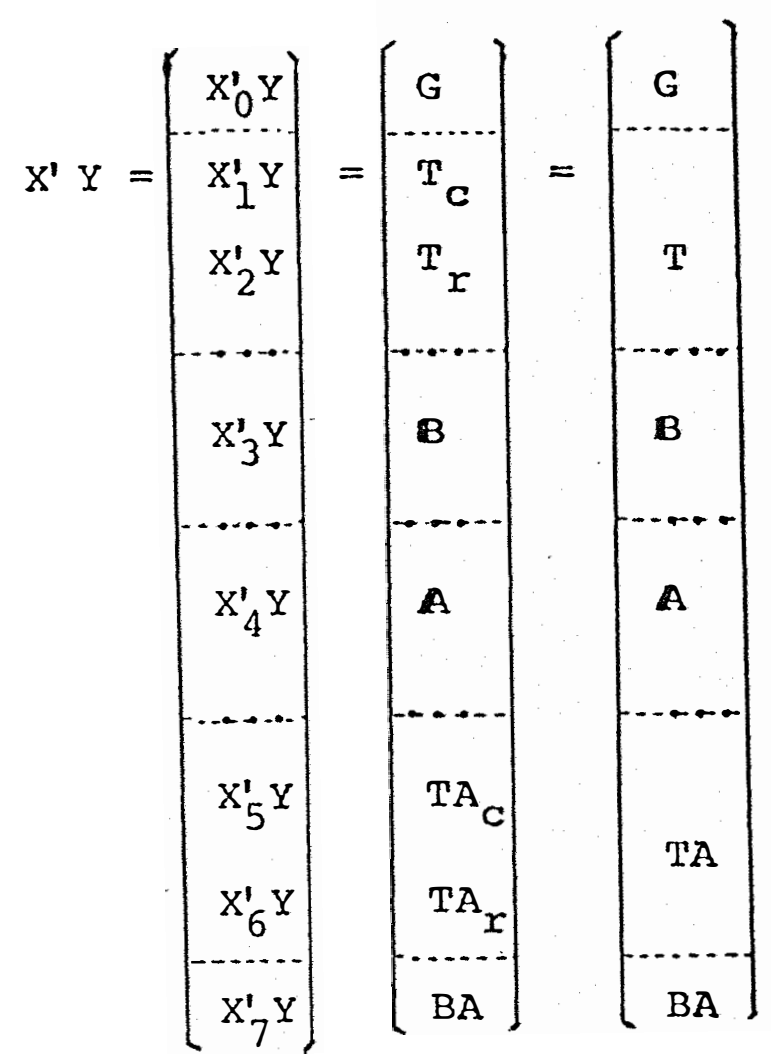

onde

$\mathbf{G}=\dot{e}$ o vetor do total geral observado, dim $1 \times 1$.

$\mathrm{T}_{\mathrm{C}}=\overline{\mathrm{e}}$ o vetor dos totais observados dos tratamentos comuns, $\operatorname{dim} c \times 1$.

$$
T_{c}^{\prime}=\left[T_{1} T_{2} \ldots T_{c}\right]
$$

$\mathrm{T}_{r}=\hat{\mathrm{e}}$ o vetor dos totais observados dos tratamentos regulares, $\operatorname{dim} z \times 1$.

$$
T_{r}^{\prime}=\left\{T_{11} \ldots T_{k 1} T_{12} \cdots T_{k 2} \cdots T_{1 b} \cdots T_{k b}\right]
$$


$B=e ́$ o.vetor dos totais observados para blocos, dim $6 \times 1$.

$$
B^{\prime}=\left[B_{1} B_{2} \cdots B_{b}\right]
$$

$A=\bar{e}$ o vetor dos totais observados para tratamentos secundá rios, dim $v \times 1$.

$$
A^{\prime}=\left[A_{1} A_{2} \ldots A_{v}\right]
$$

$\mathrm{TA}_{C}=\overline{\mathrm{e}}$ o vetor dos totais observados para as interações tratamentos comuns $\mathrm{x}$ secundários, dim cuxl.

$$
\mathrm{TA}_{\mathrm{C}}^{\prime}=\left[T A_{11} \mathrm{TA}_{12} \ldots T A_{1 v} \mathrm{TA}_{21} \ldots T A_{2 v} \ldots T A_{c 1} \ldots T A_{c v}\right]
$$

$\mathrm{TA}_{r}=\overline{\mathrm{e}}$ o vetor dos totais observados para as interações tratamentos regulares $x$ secunaários, dim zuxl.

$$
T A_{r}^{\prime}=\left[T A_{111} T A_{211} \ldots T A_{k 11} T A_{121} T A_{221} \ldots T A_{k 21} \ldots T A_{16 v^{\prime}} . T A_{k b d}\right]
$$

$\mathrm{BA}=\overline{\mathrm{e}}$ o vetor dos totais observados para as interações blo$\cos x$ tratamentos secundários, dim buxl.

$$
B A^{\prime}=\left[B A_{11} B A_{21} \ldots B A_{b 1} B A_{12} \ldots A_{b 2} \ldots B A_{1 v} \ldots B A_{b v}\right]
$$


E o sistema de equações $X^{\prime} X \hat{\theta}=X^{\prime} Y,(431)$ toma a seguinte forma.

$$
\left[\begin{array}{llllll}
n & g & C & d & e & f \\
g^{\prime} & R & N & P & S & D \\
C^{\prime} & N^{\prime} & A & K & V & F \\
d^{\prime} & P^{\prime} & K^{\prime} & U & H & G \\
e^{\prime} & S^{\prime} & V^{\prime} & H^{\prime} & L & B \\
f^{\prime} & D^{\prime} & F^{\prime} & G^{\prime} & B^{\prime} & W
\end{array}\right] \quad\left[\begin{array}{c}
\hat{m} \\
\hat{\tau} \\
\hat{b} \\
\hat{a} \\
\hat{t} a \\
\hat{b} a
\end{array}\right]=\left[\begin{array}{c}
G \\
T \\
B \\
A \\
T A \\
B A
\end{array}\right]
$$

efetuando-se os produtos e usando as restrições impostas em 4.2; temse.

$$
\begin{aligned}
& \mathrm{n} \hat{\mathrm{m}} \quad=\mathrm{G}(1) \\
& \mathrm{g}^{\prime} \hat{\mathrm{m}}+\hat{\mathrm{R}}+\hat{\mathrm{N}}+\hat{\mathrm{b}} \quad=\mathrm{T} \text { (2) } \\
& \mathrm{C}^{\prime} \hat{\mathrm{m}}+\mathrm{N}^{\prime} \hat{\tau}+\hat{A b}=\mathbf{B} \text { (3) } \\
& d^{\prime} \hat{\mathrm{m}}+U \hat{a}=A(4) \\
& e^{\prime} \hat{m}+S^{\prime} \hat{\tau}+V^{\prime} \hat{b}+H^{\prime} \hat{a}+L \hat{t a}+B \hat{b a}=T A(5) \\
& f^{\prime} \hat{m}+D^{\prime} \hat{\tau}+F^{\prime} \hat{b}+G^{\prime} \hat{a}+B^{\prime} \hat{t a}+W \hat{b} a=B A(6)
\end{aligned}
$$

4.4. Solução do Sistema de Equações

De 43.2 , obtemos

$$
\hat{m}=\frac{G}{n} \quad(44.1)
$$

de (3), isolando-se o valor de $\hat{b}$, tèm-se

$$
\begin{aligned}
& \hat{b}=A^{-1}\left[B-N^{\prime} \hat{\tau}-C^{\prime} \hat{m}\right] \\
& \hat{b}=\frac{1}{v K}\left[B-N^{\prime} \hat{\tau}-C^{\prime} \hat{m}\right]
\end{aligned}
$$




$$
\begin{aligned}
& {\left[\mathrm{R}-\mathrm{NA}^{-1} \mathrm{~N}^{\prime}\right] \hat{\tau}=\mathrm{T}-\mathrm{NA} A^{-1} \mathrm{~B}-\hat{\mathrm{m}}\left[\mathrm{g}^{\prime}-\mathrm{NA}^{-1} \mathrm{C}^{\prime}\right]} \\
& \text { mas } \mathrm{g}^{\prime}=\mathrm{NA}^{-1} \mathrm{C}^{\prime} \quad \text { e fazendo-se } \\
& \mathbf{C}=\mathrm{R}-\mathrm{NA}^{-1} \mathrm{~N}^{\prime}=\mathrm{R}-\frac{1}{v K} \mathrm{NN}^{\prime} \quad(44.5) \\
& Q=\mathrm{T}-\mathrm{NA}^{-1} \mathrm{~B}=\mathrm{T}-\frac{1}{v K} \mathrm{NB} \quad(44.6) \\
& \text { assim (44.4) fica } \\
& \hat{C} \hat{\tau}=Q \quad(44.7)
\end{aligned}
$$

onde a matriz Q é partida da seguinte forma

$$
\begin{aligned}
& Q=\left[\begin{array}{c}
Q_{C} \\
Q_{r}
\end{array}\right] ; \quad Q_{C}=\begin{array}{c}
\text { submatriz associada aos tratamentos comuns, dim } \\
c \times l, \text { e cujos elementos são }
\end{array} \\
& Q_{i}=T_{i}-\frac{1}{R} G \\
& Q_{r}=\text { submatriz associada aos tratamentos regulares } \\
& \operatorname{dim}(z \times 1) \text { e cujos elementos são: } \\
& 2_{i^{\prime} j}=T_{i^{\prime} j}-\frac{1}{K} \dot{j}_{i^{\prime} j}
\end{aligned}
$$

onde

$\dot{j}_{i^{\prime} j}=B_{j}$, pois não se tem repetições de tratamentos regulares assim

$$
Q_{i \prime j}=T_{i \prime j}-\frac{1}{K} B_{j}
$$


Como a matriz $\boldsymbol{c}$ è uma matriz singular de caracterís tica $\ell-1=(c+z-1)$. Desse modo, e como em PIMENTEL GOMES (1967), adotou-se uma matriz $\mathbf{z}$ de restrição e de característica 1 , com. dimensão $(c+z) \times(c+z)$ e tal que $\mathbf{z} \hat{\tau}=\dot{\phi}$,

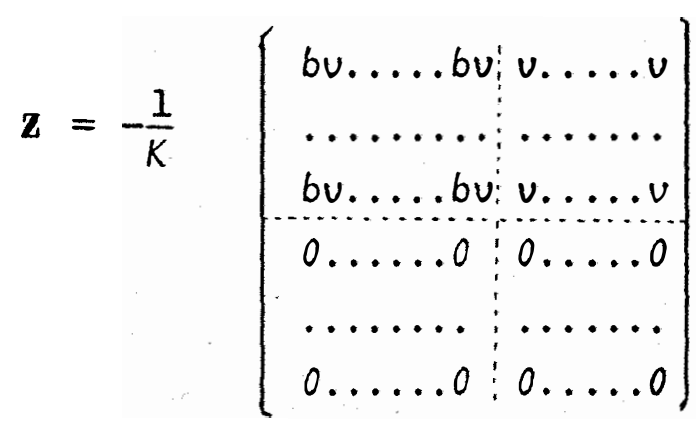

assim

$$
\begin{aligned}
\hat{\mathbf{C}} \hat{\tau} & =Q \\
-\frac{\mathbf{Z} \hat{\tau}}{\grave{\hat{\tau}}} & =\phi \\
(\boldsymbol{C}-\mathbf{Z}) & =Q
\end{aligned}
$$

fazendo $(\boldsymbol{C}-\mathrm{Z})=\mathbf{M}$, termse

$$
\begin{aligned}
& \mathbf{M} \hat{\tau}=Q \\
& \therefore \hat{\tau}=M^{-1} Q
\end{aligned}
$$


onde, $M^{-1}$, tem a seguinte forma

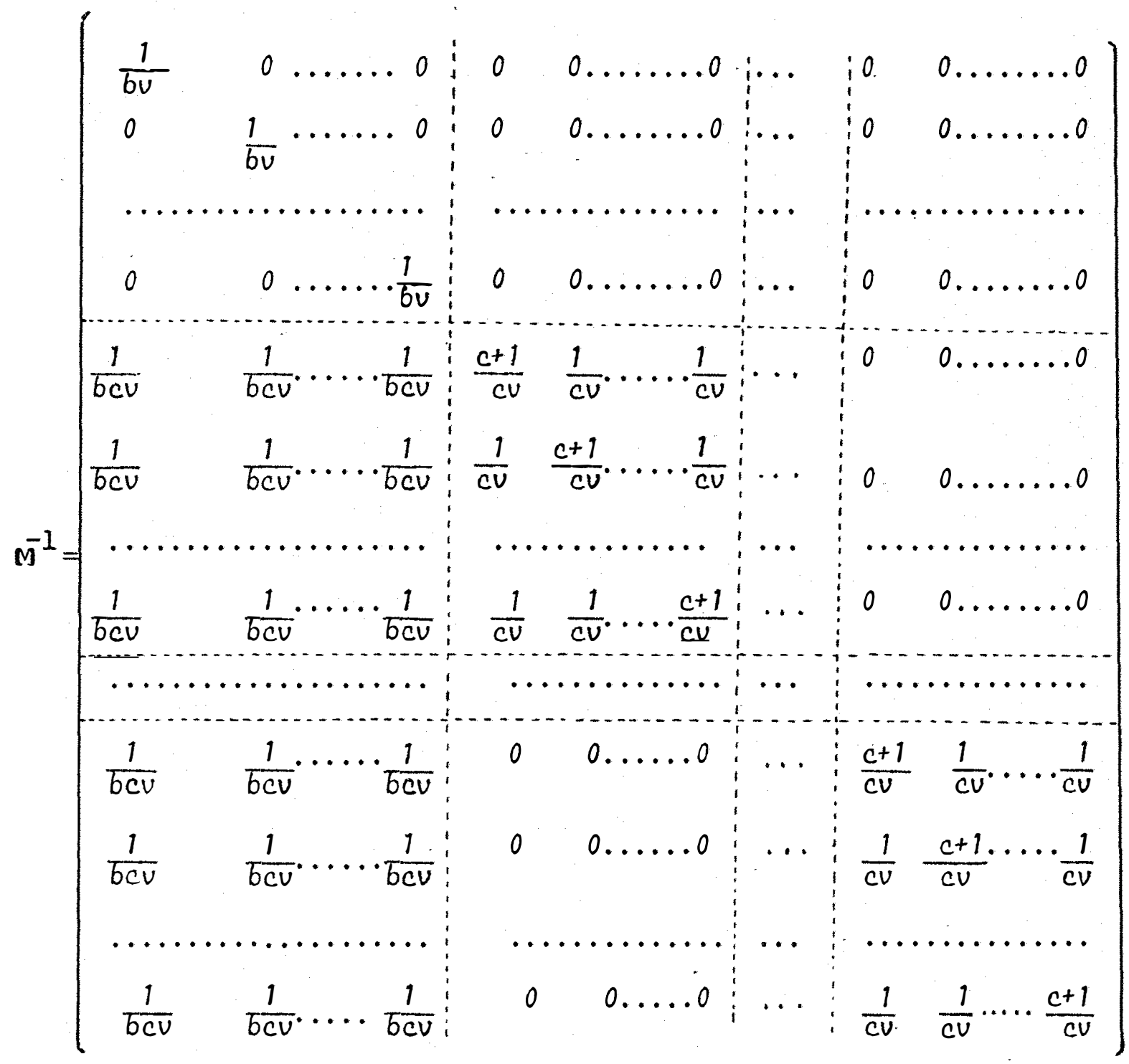

assim uma solução para um determinado tratamento principal comum, $i$, è dada por

$$
\hat{t}_{i}=\frac{1}{b v} Q_{i}=\frac{1}{b v} T_{i}-\hat{m}
$$

e para um detenminado tratamento principal regular $i^{\prime j}$ 


$$
z_{i^{\prime} j}=\frac{1}{v} Q_{i^{\prime} j^{+}}+\frac{1}{c v} \sum_{i^{\prime}}^{k} Q_{i^{\prime} j}+\frac{1}{b c v}{\underset{i}{\sum}}_{i}^{c} Q_{i}
$$

outra forma de apresentar (44.13), usando somente os totais de tratamentos, è obtido substituindo os valores do $2^{\prime} s$, ou seja. $\left.z_{i^{\prime} j}=\frac{1}{v}\left[T_{i^{\prime} j}-\frac{\dot{j}_{i^{\prime} j}}{K}\right]+\frac{1}{c v} \sum_{i^{\prime}}^{k}\left[T_{i^{\prime} j}-\frac{j_{i^{\prime} j}}{K}\right]+\frac{1}{b c v} \stackrel{\Sigma}{i}_{i}^{c} T_{i}-\frac{G}{K}\right]$ como $j_{i^{\prime} j}=B_{j}$, tem-se $z_{i^{\prime} j}=\frac{T_{i^{\prime} j}}{v}-B_{j}\left(\frac{1}{K v}+\frac{k}{c K v}\right)+\frac{1}{c v} \sum_{i^{\prime}}^{k} T_{i^{\prime} j}+\frac{1}{b c v} \sum_{i} T_{i}-\frac{G}{b K v}$ como $B_{j}=\sum_{i}^{k} T_{i}^{\prime} j^{+} \stackrel{c}{\left(\Sigma^{*}\right.} T_{i}^{\prime}{ }_{[\text {dentro de } j]}$ e $K=c+k$, sendo $\left.\left.\stackrel{c}{\left(\Sigma^{*}\right.} T_{i}\right)_{[\text {dentro de } j]}=\stackrel{c}{\Sigma^{*}} T_{i}\right) / j$ é a soma dos tratamentos comuns no bloco $B_{j}$.

$\left.\left.\therefore \hat{t}_{i^{\prime} j}=\frac{T_{i^{\prime} j}}{v}-\underset{i^{\prime}}{k} T_{i^{\prime} j}+\underset{i}{\left(\Sigma^{*}\right.} T_{i}\right) / j\right)\left(\frac{c+k}{c K v}\right)+\frac{1}{c v} \sum_{i^{\prime}} T_{i^{\prime} j}+\frac{1}{b c v} \sum_{i} T_{i}-\frac{G}{b K v}$ $\hat{t}_{i^{\prime} j}=\frac{T_{i^{\prime} j}^{v}}{v}+\left(\frac{1}{c v}-\frac{1}{c v}\right) \sum_{i^{\prime}}^{k} T_{i^{\prime} j}-\frac{1}{c v}\left(\sum_{i}^{*} T_{i}\right) / \dot{j}+\frac{1}{b c v} \sum_{i}^{\Sigma} T_{i}-\hat{m}$ $\hat{t}_{i j j}=\frac{T_{i j} j}{v}-\frac{1}{c v}\left(\Sigma_{i}^{*} T_{i} / j+\frac{1}{b c v} \sum_{i} T_{i}-\hat{m}\right.$ $(4414)$ De $(442)$

$$
\begin{aligned}
& \hat{b}=A^{-1}\left[B-N^{\prime} \hat{\tau}-c^{\prime} \hat{m}\right], \text { tem-se } \\
& \hat{b}_{j}=\frac{1}{c v}\left(\Sigma_{i}^{*} T_{i}\right) / j-\frac{1}{b c v} \sum_{i} T_{i}
\end{aligned}
$$


Da equação (4), tem-se

$$
\begin{aligned}
& \hat{a}=U^{-1}\left[A-d^{\prime} \hat{m}\right] \quad(44.16) . \\
& \left.\hat{a}=U^{-1} A-b K \hat{m} E_{(v \times 1)}\right] \quad(44.17)
\end{aligned}
$$

que é uma solução para os tratamentos secundários, assim para um determinado, $\hat{a}_{v}$, tem-se

$$
\hat{a}_{v}=\frac{A v}{b K}-\hat{m}
$$

na equação (5) temos

$$
\hat{t} a=L^{-1}\left[T A-B \hat{b} a--H^{\prime} \hat{a}-V^{\prime} \hat{b}-S^{\prime} \hat{\tau}-e^{\prime} \hat{m}\right]
$$

substituindo (44.19) na equação (6) tem-se

$$
\begin{aligned}
& \left(f^{\prime}-B^{\prime} L^{-1} e^{\prime}\right) \hat{m}+\left(D^{\prime}-B^{\prime} L^{-1} S^{\prime}\right) \hat{\tau}+\left(F^{\prime}-B^{\prime} L^{-1} V^{\prime}\right) \hat{b}+ \\
& +\left(G^{\prime}-B^{\prime} L^{-1} H^{\prime}\right) \hat{a}+\left(B^{\prime} L^{-1} T A\right)+\left(W-B^{\prime} L^{-1} \hat{B}\right) \quad b a=B A
\end{aligned}
$$

mas $f^{\prime}=B^{\prime} L^{-1} e^{\prime} ; \quad D^{\prime}=B^{\prime} L^{-1} S^{\prime} ; \quad G^{\prime}=B^{\prime} L^{-1} H^{\prime}$

$\therefore \quad\left(W-B^{\prime} L^{-1} B\right) \hat{b} a=B A-\left(F^{\prime}-B^{\prime} L^{-1} V^{\prime}\right) \hat{b}-B^{\prime} L^{-1} T A \quad(44.20)$

de acordo com as restrições impostas, $\underset{j}{\Sigma} \hat{b} a_{j v}=0$ e $\underset{j}{\sum \hat{b}_{j}}=0$; tem se 


$$
\begin{aligned}
& \left(W-B^{\prime} L^{-1} B\right) \hat{b} a=c \hat{b} a \quad e \\
& \left(F^{\prime}-B^{\prime} L^{-1} V^{\prime}\right) \hat{b}=c \psi \hat{b} \text { onde } \\
& \psi=\left[\begin{array}{c}
I(b) \\
\cdots \cdots \cdot \cdot \\
\hdashline I_{(b)}
\end{array}\right]_{(b v \times b)}
\end{aligned}
$$

assim (44.20), pode ser escrita da seguinte forma

$$
\begin{aligned}
& c \hat{b a}=B A-c \psi \hat{b}-B^{\prime} L^{-1} T A \\
& \hat{b a}=\frac{B A}{c}-\psi \hat{b}-\frac{I}{c} B^{\prime} L^{-1} T A
\end{aligned}
$$

que é uma solução para a interação blocos $\mathrm{x}$ anos, assim para um determinado $\hat{b a}_{j v^{\prime}}$ tem-se

$\hat{b} a_{j v}=\frac{1}{c} B A_{j v}-\hat{b}_{j}-\frac{1}{b c} \sum_{i}^{\sum} T A_{i v}-\frac{1}{c} \sum_{i^{\prime}} T A_{i^{\prime} j v}$

substituindo $\hat{b}_{j}$, dado em (44.15) tem-se

$\hat{b} a_{j v}=\frac{1}{c} B A_{j v}-\frac{1}{c v}\left(\sum_{i}^{*} T_{i}{ }^{\prime} / j+\frac{1}{b c v} \sum_{i}^{\Sigma T} T_{i}-\frac{1}{b c} \underset{i}{\Sigma} T A_{i v}-\frac{1}{c} \sum_{i^{\prime}}^{\Sigma T A_{i} j v}\right.$

substituindo em 44.19, o valor de ba dado em (44.21) tem-se

$$
\begin{aligned}
\left.\hat{t a}=L^{-1}+\frac{1}{c} L^{-1} B B^{\prime} L^{-1}\right) T A & -\frac{1}{c} L^{-1} B B A+\left(L^{-1} B \psi-L^{-1} V^{\prime}\right) \hat{b}-L^{-1} H^{\prime} \hat{a}- \\
& -L^{-1} S^{\prime} \hat{\tau}-L^{-1} e^{\prime} \hat{m}
\end{aligned}
$$

$\operatorname{mas} L^{-1} B=L^{-1} V^{\prime}$ 
e substituindo os valores de $\hat{\tau}$ e $\hat{a}$, dados em (44.11) e (44.16) respectivamente

$$
\begin{aligned}
& \hat{t a}=\left[L^{-1}+\frac{1}{c} L^{-1} B^{\prime} L^{-1}\right] T A-\frac{1}{c} L^{-1} B B A-L^{-1} H^{\prime} U^{-1} A-L^{-1} S^{\prime} M^{-1} Q+ \\
& \quad+\left[L^{-1} H^{\prime} U^{-1} d^{\prime}-L^{-1} e^{\prime}\right] \hat{m} \\
& \text { mas } L^{-1} H^{\prime} U^{-1} d^{\prime}=L^{-1} e^{\prime}, \\
& \therefore \quad t a=\left[L^{-1}+\frac{1}{c} L^{-1} B B^{\prime} L^{-1}\right] T A-\frac{1}{c} L^{-1} B B A-L^{-1} H^{\prime} U^{-1} A-L^{-1} S^{\prime} M^{-1} Q \\
& \quad \ldots(4424)
\end{aligned}
$$

que é uma solução para a interação tratamento principal $x$ anos onde: 
.35 .

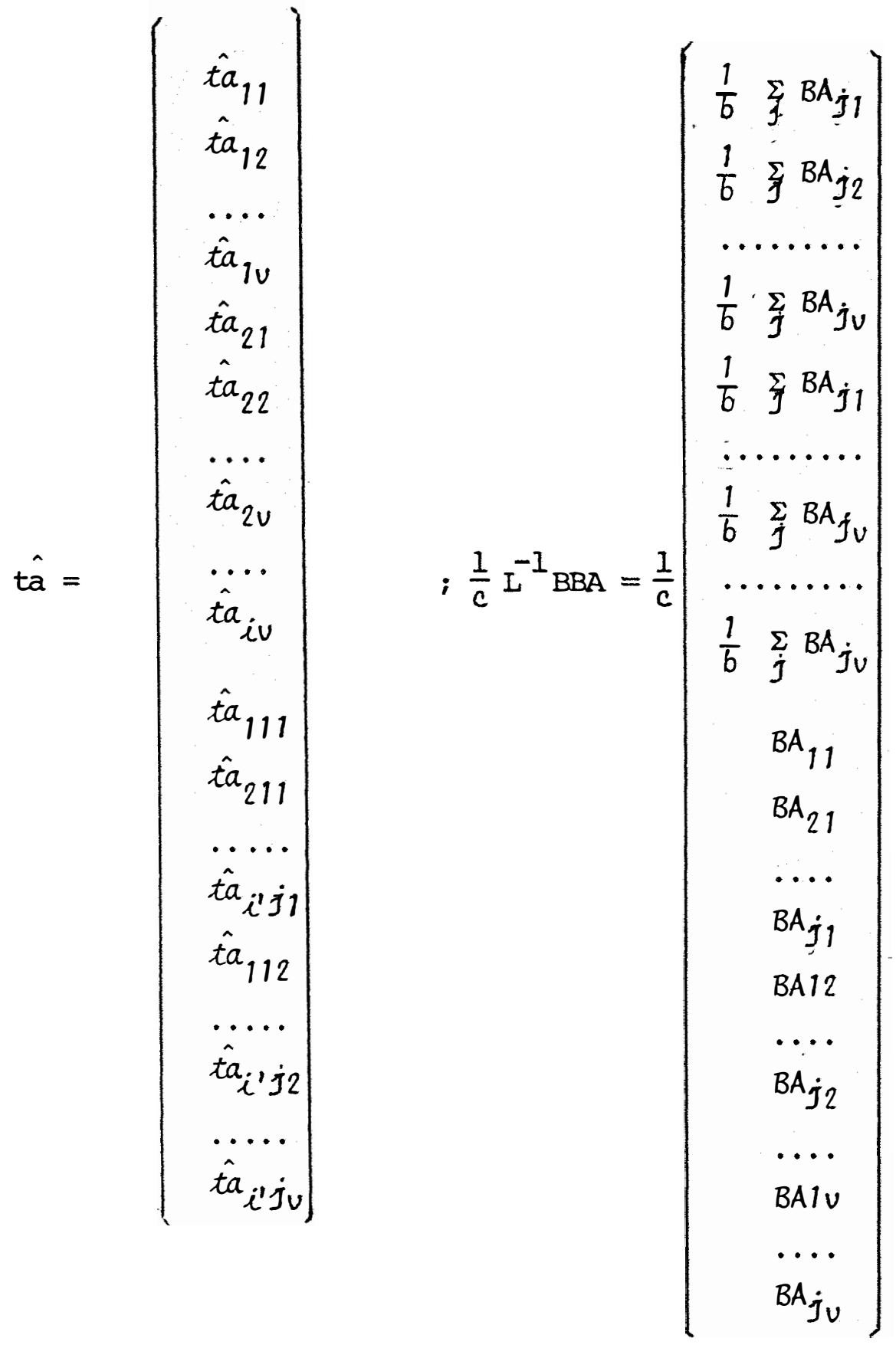


$\left[I^{-1}+\frac{1}{C} L^{-1} B^{\prime} L^{-1}\right] T A=$

\begin{tabular}{|c|c|c|c|}
\hline$\frac{1}{b} T A_{11}+\frac{1}{c b}$ & $\sum_{i} T A_{i 1}+\frac{1}{c^{b}} \quad \sum_{i j} T A_{i^{\prime} j 1}$ & & $\frac{1}{b} T A_{11}+\frac{1}{c b} A_{1}$ \\
\hline$\frac{1}{b} T A_{12}+\frac{1}{c b}$ & $\sum_{i} T A_{i 2}+\frac{1}{c b} \sum_{i^{\prime} j} T A_{i^{\prime} j 2}$ & & $\frac{1}{b} T A_{12}+\frac{1}{c b} A_{2}$ \\
\hline$\frac{1}{b} T A_{1 v}+\frac{1}{c b}$ & $\sum_{i} T A_{i v}+\frac{1}{c b} \sum_{i^{\prime} j} T A_{i j v}$ & & $\frac{1}{b} T A_{1 v}+\frac{1}{c b} A_{v}$ \\
\hline$\frac{1}{b} T A_{21}+\frac{1}{c b}$ & $\sum_{i} T A_{i 1}+\frac{1}{c b} \sum_{i^{\prime} j} T A_{i^{\prime} j 1}$ & & $\frac{1}{b} T A_{21}+\frac{1}{c b} A_{1}$ \\
\hline$\frac{1}{b} T A_{2 v}+\frac{1}{c b}$ & $\sum_{i} T A_{i v}+\frac{1}{c b} \sum_{i^{\prime j}} T A_{i^{\prime} j v}$ & & $\frac{1}{b} T A_{2 v}+\frac{1}{c b} A_{v}$ \\
\hline$\frac{1}{b} T A_{i v+} \frac{1}{c b}$ & $\sum_{i} T A_{i v}+\frac{1}{c b} \sum_{i^{\prime j}} T A_{i j}{ }^{\prime}$ & $1=$ & $\frac{1}{b} T A_{i v}+\frac{1}{c b} A_{v}$ \\
\hline $\mathrm{TA}_{111}+\frac{1}{\mathrm{cb}}$ & $\sum_{i} T A_{i 1}+\frac{1}{c} \quad \sum_{i^{\prime}} T A_{i 11}$ & & $T A_{111}+\frac{1}{c b} \sum_{i} T A_{i 1}+\frac{1}{c} \sum_{i} T A_{i^{\prime} 11}$ \\
\hline$T A_{211}+\frac{1}{c b}$ & $\sum_{i} T A_{i 1}+\frac{1}{\dot{c}} \quad \sum_{i^{\prime}} I A_{i} 11$ & & $T A_{211}+\frac{1}{c b} \cdot \sum_{i} T A_{i 1}+\frac{1}{c} \sum_{i^{\prime}} T A_{i^{\prime} 11}$ \\
\hline$T_{i^{\prime} j i}+\frac{1}{c b}$ & $\sum_{i} T A_{i 1}+\frac{1}{c} \sum_{i} T A_{i j 1}$ & & $T A_{i^{\prime} j 1}+\frac{i}{c b} \sum_{i}^{\sum} T A_{i 1}+\frac{1}{c} \sum_{i^{\prime}}^{T_{1}} T A_{i^{\prime} j 1}$ \\
\hline$T A_{112}+\frac{1}{c b}$ & $\begin{array}{lll}\Sigma & T A_{i 2}+\frac{1}{c} & \Sigma \\
i & T A^{\prime} 12 & \end{array}$ & & $T A_{112}+\frac{1}{c b} \sum_{i}^{\sum} T A_{i 2}+\frac{1}{c} \sum_{i^{\prime}} T A_{i 12}$ \\
\hline$T A_{i \prime j 2}+\frac{i}{c b}$ & $\sum_{i} T A_{i 2}+\frac{i}{c} \quad \sum_{i} T A_{i j 2}$ & & $T A_{i j 2}+\frac{i}{c b} \sum_{i}^{\Sigma T A_{i 2}}+\frac{i}{c} \sum_{i^{\prime}}^{\Sigma} T A_{i^{\prime} j 2}$ \\
\hline$T_{i^{\prime} j_{v}}+\frac{i}{c b}$ & $\sum_{i}^{\Sigma} T A_{i v}+\frac{i}{c} \sum_{i} T A_{i j v}$ & & $T A_{i j} j v+\frac{i}{c b} \sum_{i}^{\Sigma} T A_{i v}+\frac{i}{c} \sum_{i^{\prime}}^{\Sigma} T A_{i^{\prime} j u}$ \\
\hline
\end{tabular}




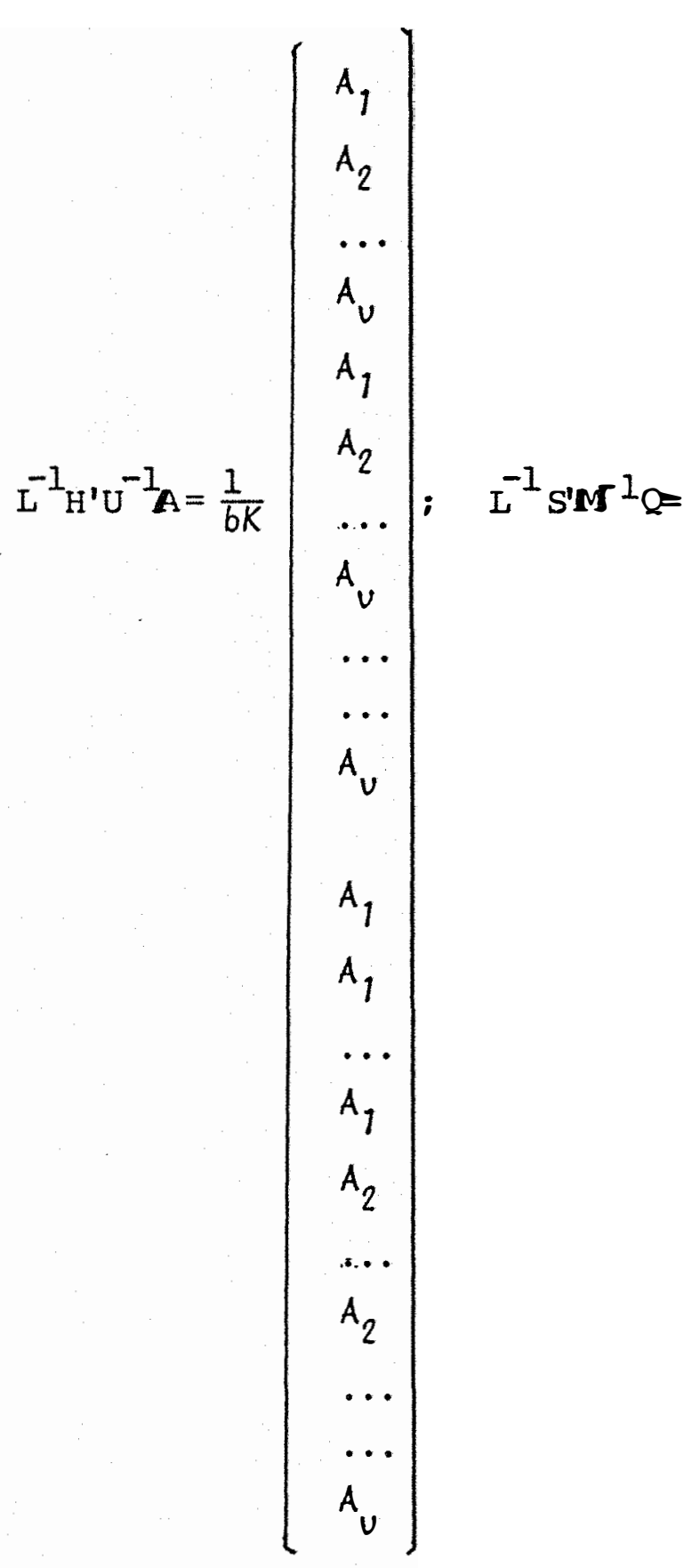

$$
\begin{array}{ll}
\frac{1}{b v} & 21 \\
\frac{1}{b v} & 21
\end{array}
$$$$
\frac{1}{6 v} 2
$$$$
\frac{1}{6 v} \quad 2_{2}
$$$$
\frac{1}{\text { bu }} 2
$$$$
\frac{1}{b v} \quad 2
$$$$
\frac{1}{b v} 2_{i}
$$$$
\frac{1}{b c v} \sum_{i} 2_{i}+\frac{1}{v} 2_{11}+\frac{1}{c v} \sum_{i^{\prime}} Q_{i^{\prime} 1}
$$$$
\frac{1}{b c v} \sum_{i} 2_{i}+\frac{1}{v} 2_{21}+\frac{1}{c v} \sum_{i^{\prime}} 2_{i^{\prime} 1}
$$$$
\frac{1}{b c v} \sum_{i} 2_{i}+\frac{1}{v} Q_{i^{\prime} 1}+\frac{1}{c v} \sum_{i^{\prime}} Q_{i^{\prime} 1}
$$$$
\frac{1}{b c v} \sum_{i} 2_{i}+\frac{1}{v} 2_{12}+\frac{1}{c v} \sum_{i^{\prime}} 2_{i^{\prime} 2}
$$$$
\frac{1}{b c v} \sum_{i} 2_{i}+\frac{1}{v} 2_{i^{\prime} 2}+\frac{1}{c v} \sum_{i^{\prime}} 2_{i^{\prime} 2}
$$$$
\frac{1}{b c v} \sum_{i} Q_{i}+\frac{1}{v} Q_{i^{\prime} j}+\frac{1}{c v} \sum_{i^{\prime}} Q_{i^{j} j}
$$ 
, 38.

assim para as interações tratamentos comuns $x$ anos, temrse

$$
\begin{aligned}
& \hat{t a}_{i v}=\frac{1}{b} T A_{i v}+\frac{1}{b c} \sum_{i} T A_{i v}+\frac{1}{b c} \sum_{i j} T A_{i \prime j} v^{-\frac{1}{b c}}{ }^{B A} A_{j v}-\frac{1}{b K} A_{v}-\frac{1}{b v} Q_{i} \\
& \text { mas } \sum_{j} B A_{j v}=\sum_{i} T A_{i v}+\sum_{i j} T A_{i \prime j v} \\
& \therefore \hat{t a}_{i v}=\frac{1}{b} T A_{i v}-\frac{1}{b R} A_{v}-\frac{1}{b v} Q_{i} \quad(44.25) \\
& \text { ou substituindo } Q_{i}=T_{i}-\frac{G}{K}, \text { tem-se } \\
& \hat{t a}_{i v}=\frac{1}{b} T A_{i v}-\frac{1}{b R} A_{v}-\frac{1}{b v} T_{i}+\frac{G}{b K v} \\
& \hat{t a}_{i v}=\frac{1}{b} T A_{i v}-\frac{1}{b K} A_{v}-\frac{1}{b v} T_{i}+\hat{m}
\end{aligned}
$$

e para as interações tratamentos regulares $\mathrm{x}$ anos, tem-se

$$
\begin{aligned}
\hat{t a}_{i^{\prime} j v} & =T A_{i^{\prime} j u}+\frac{1}{c b} \sum_{i} T A_{i v}+\frac{1}{c} \sum_{i^{\prime}} T A_{i^{\prime} j u}-\frac{1}{b K} A_{v}-\frac{1}{b c v} \sum_{i} 2_{i}- \\
& -\frac{1}{v} Q_{i^{\prime} j}-\frac{1}{c v} \sum_{i^{\prime}} 2_{i^{\prime} j}-\frac{1}{c}{ }_{j u} A_{j v}
\end{aligned}
$$

ou substituindo os valores dos $Q^{\prime}$ s pelos seus valores dados em 44.8 e 44.10 respectivamente, tem-se

$$
\begin{aligned}
& \hat{t a}_{i^{\prime} j v}=T A_{i^{\prime} j v}+\frac{1}{c b} \sum_{i} T A_{i v}+\frac{1}{c} \sum_{i^{\prime}} T A_{i^{\prime} j u}-\frac{1}{c} B A_{j v}-\frac{1}{b K} A_{v}- \\
& -\frac{1}{b c v} \sum_{i}\left[T_{i}-\frac{G}{K}\right]-\frac{1}{v}\left[T_{i^{\prime} j}-\frac{1}{K} B_{j}\right]-\frac{1}{c v} \sum_{i^{\prime}}^{k}\left[T_{i j}-\frac{1}{K} B_{j}\right] \\
& \hat{t a}_{i^{\prime} j v}=T A_{i^{\prime} j v}+\frac{1}{c b} \sum_{i} T A_{i v}+\frac{1}{c} \sum_{i^{\prime}} T A_{i^{\prime} j v}-\frac{1}{c} B A_{j v}-\frac{1}{b K} A_{v}- \\
& -\frac{1}{b c V} \quad \sum_{i} T_{i}+\frac{c G}{b c v R}-\frac{1}{v} T_{i^{\prime} j}-\frac{1}{c V} \quad \sum_{i^{\prime}}^{T^{\prime}} i_{i^{\prime} j}+\left(\frac{c+k}{c K \bar{v}}\right) B_{j}
\end{aligned}
$$


mas

$$
\begin{aligned}
& B_{j}=1 \sum_{i}^{\Sigma} T_{i} / / j+\sum_{i} T_{i^{\prime} j} e \\
& B A_{j v}=1 \sum_{i}^{*} T A_{i v} / / j+\sum_{i^{\prime}} T A_{i v}
\end{aligned}
$$

substituindo estes valores em 4427, resulta

$$
\begin{aligned}
& \hat{t a}_{i j v}=T A_{i j} j v+\frac{1}{c b} \sum_{i} T A_{i v}-\frac{1}{c}\left(\sum_{i}^{*} T A_{i v}\right) / j-\frac{1}{b K} A_{v}-\frac{1}{b c v} \sum_{i} T_{i}{ }^{-} \\
& -\frac{l}{v} T_{i \prime j}+\frac{l}{c v} l \quad \sum_{i}^{*} T_{i} / / j+\hat{m}
\end{aligned}
$$




\subsubsection{Introdução}

Do modelo matemático $\mathrm{Y}=\mathrm{X} \theta+\varepsilon$, e da estimativa de $\theta$, resulta a soma de quadrados dos residuos

$$
\begin{aligned}
\text { SQ Res } & =Y^{\prime} Y-\hat{\theta}^{\prime} X^{\prime} Y \\
& =\text { SQTOtal - SQ Parâmetros = SQT-SQP }
\end{aligned}
$$

$\operatorname{mas} X^{\prime} Y=X^{\prime} X \hat{\theta}$

$\therefore S Q \operatorname{Res}=Y^{\prime} Y-\hat{\theta}^{\prime} X^{\prime} X \hat{\theta}$

onde

$$
\mathrm{SQP}=\hat{\theta} X^{\prime} X \hat{\theta}=\hat{\theta} X^{\prime} Y \quad(4512)
$$

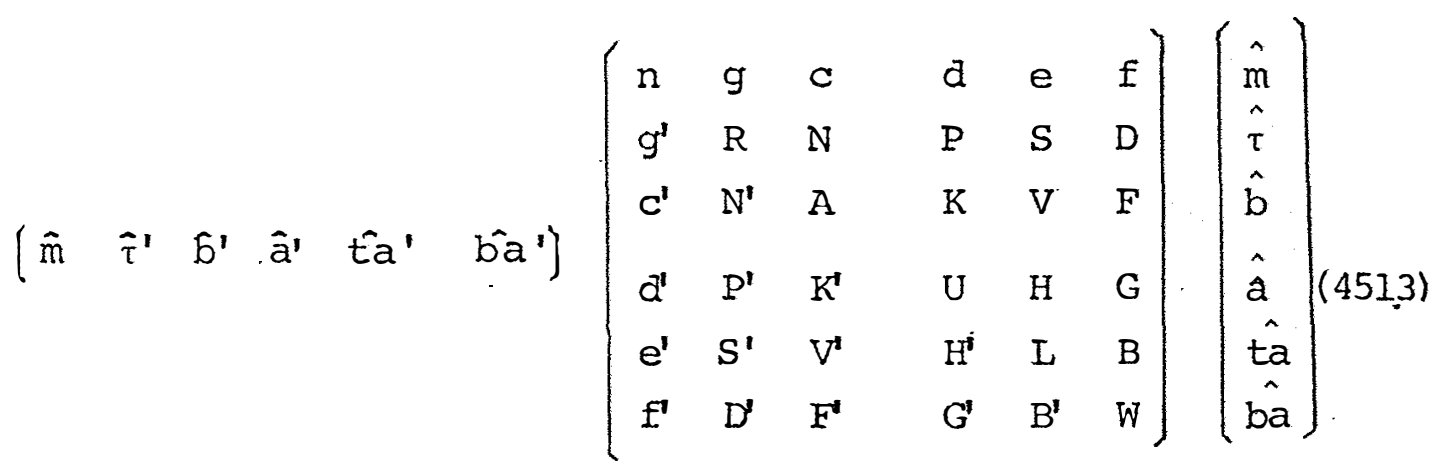


usando-se as notações de IEMMA(1981) tem-se para as partições das matrizes

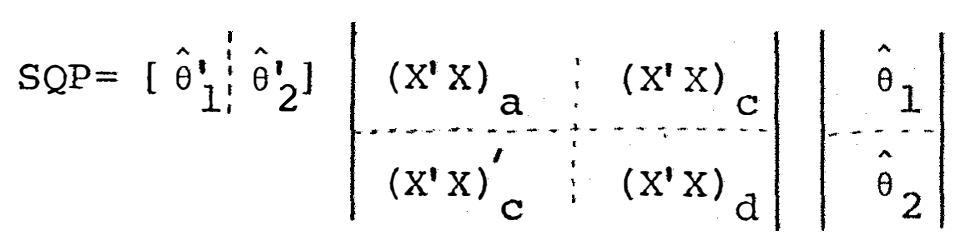

onde as partições de $\hat{\theta}$, referem-se às duas partes encontrdatas no esquema de anālise de variância para ensaios em parcelas subdivididas em esquema de blocos casualizados. Efetuando-se o produto tem-se

$$
\mathrm{SQP}=\hat{\theta}_{1}^{\prime}\left(X^{\prime} X\right)_{\mathrm{a}} \hat{\theta}_{1}+\hat{\theta}_{2}^{\prime}\left(X^{\prime} X\right)_{\mathrm{d}} \hat{\theta}_{2}+\hat{\theta}_{1}^{\prime}\left(X^{\prime} X\right)_{\mathrm{c}} \hat{\theta}_{2}+\hat{\theta}_{2}^{\prime}\left(X^{\prime} X\right)_{\mathrm{c}}^{\prime} \hat{\theta}_{1}
$$

mas de acordo com as restrições impostas em 4.2.tem-se

$$
\begin{aligned}
\hat{\theta}_{1}^{\prime}\left(X^{\prime} X\right)_{C} \hat{\theta}_{2}=\hat{\theta}_{2}^{\prime}\left(X^{\prime} X\right)_{c}^{\prime} & \hat{\theta}_{1}=\phi \\
S Q P & =\hat{\theta}_{1}^{\prime}\left(X^{\prime} X\right)_{a} \hat{\theta}_{1}+\hat{\theta}_{2}^{\prime}\left(X^{\prime} X\right)_{d} \hat{\theta}_{2} \\
& =\operatorname{SQP}(a)+\operatorname{SQP}(b)
\end{aligned}
$$

assim

SQReS $=$ SQT $-S Q P(a)-S Q P(b)$

ou ainda

SQRes = [ SQParcelas- SQP(a)] + [ SQT- SQParcelas-SQP (b)] (451.4) $=\operatorname{SQReS}(a)+\operatorname{SQReS}(b)$ 
onde

SQParcelas $=\frac{1}{v} \quad Y^{\prime} \Phi Y-$ Co

(451.5)

sendo

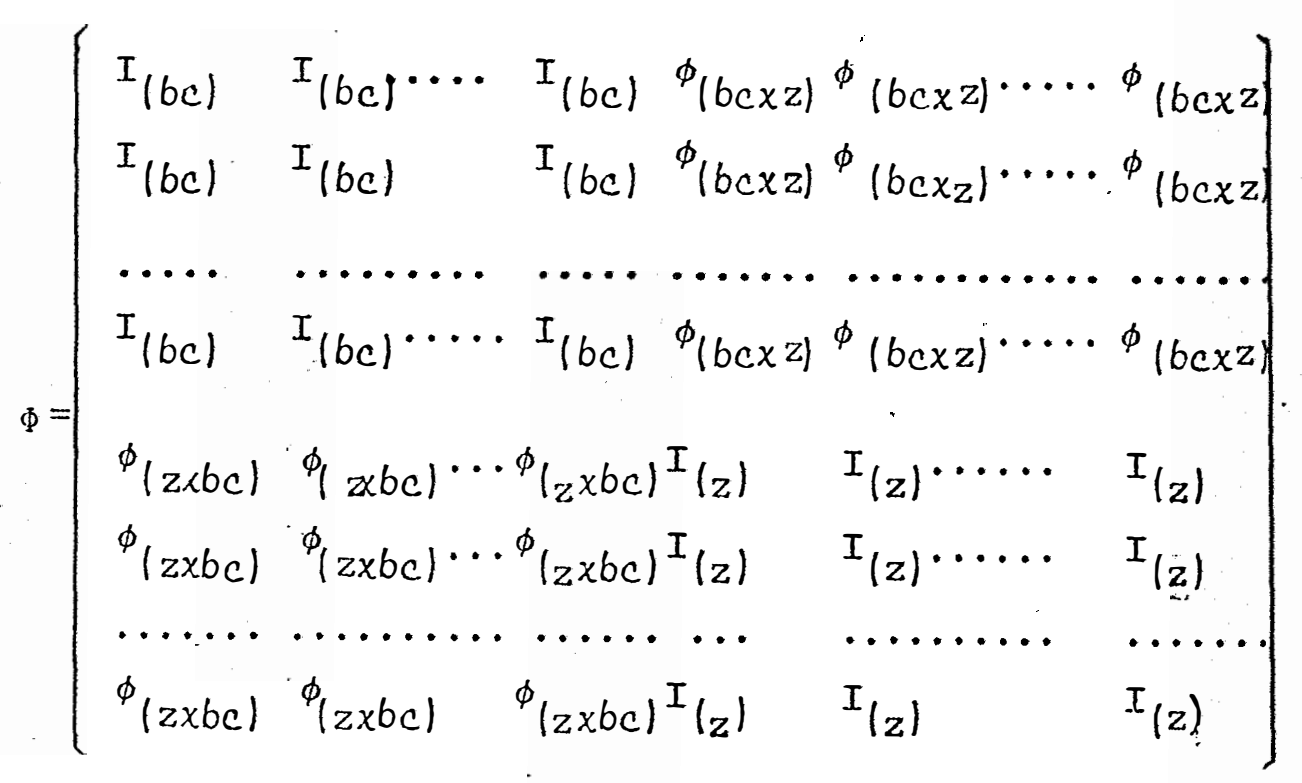

$(b c u+u z) x$. $x(b c u+v z)$

obs: a característica de $\Phi=b c+z$, mas $z=b K$, portanto a carac teristica de $\Phi=b c+b k=b K$

a SQParcelas é usualmente encontrada na literatura

$$
\text { SQParcelas }=\frac{1}{v} \quad \sum_{\ell J}\left(y_{\ell j j^{\prime}}\right)^{2}-C_{0}
$$

sendo Co $=\frac{G^{2}}{n}$ 
4.5.2. Somá de Quadrados dos Parâmetros;

Tem-se de $(45,1.2)$

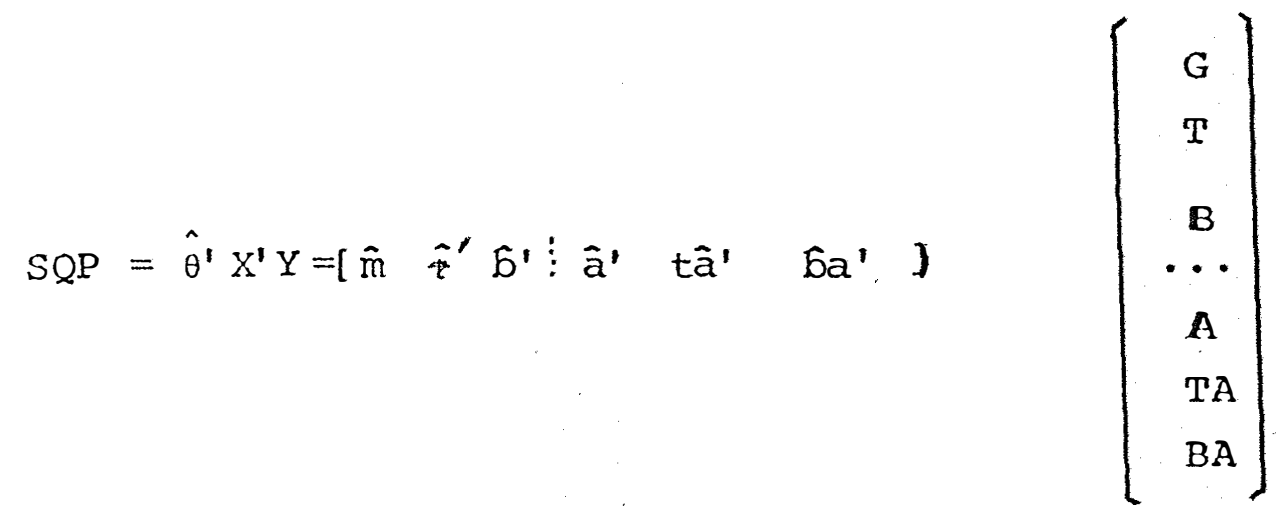

$S Q P=\left[\hat{m G}+\hat{\tau}^{\prime} T+\hat{b} B\right]+\left[\hat{a} A+t \hat{a}^{\prime} T A+\hat{b}^{\prime} B A\right]$

onde

$\operatorname{SQP}(a)=\left[\hat{m} G+\hat{\tau}^{\prime} T+\hat{b}^{\prime} B\right]$

substituindo o valor $\hat{b}^{\prime}$ de 44.2 , tem-se

$\operatorname{SQP}(a)=\hat{m} G+\hat{\tau}^{\prime} T+\left[B^{\prime} A^{-1}-\hat{r}^{\prime} N A^{-1}-\hat{m} c A^{-1}\right] B$.

$\mathrm{SQP}(\mathrm{a})=\hat{\mathrm{m} G}+\hat{\tau} \cdot[\mathrm{T}-\mathrm{NA}-1 \mathrm{~B}]+\mathrm{B}^{\prime} \mathrm{A}^{-1} \mathrm{~B}-\hat{\mathrm{mCA}}{ }^{-1} \mathrm{~B}$

mas

$$
\begin{aligned}
& \mathrm{CA}^{-1} \mathrm{~B}=\sum_{j} B_{j}=\mathrm{G} \quad \mathrm{e} \\
& \mathrm{T}^{-N A^{-1} \mathrm{~B}}=\mathrm{Q} \\
& \therefore \operatorname{SQP}(\mathrm{a})=\hat{\mathrm{mG}}+\hat{\tau}^{\prime} \mathrm{Q}+\mathrm{B}^{\prime} \mathrm{A}^{-1} \mathrm{~B}-\hat{\mathrm{m} G}
\end{aligned}
$$


como $\hat{m G}=$ Co, tem-se

$\operatorname{SQP}(a)=\mathrm{CO}+\hat{\tau}^{\prime} Q+\mathrm{B}^{\prime} \mathrm{A}^{-1} \mathrm{~B}-\mathrm{CO}(452,3)$

fazendo

a) $\mathrm{SQBIOCOS}=\mathrm{B}^{\prime} \mathrm{A}^{-1} \mathrm{~B}-\mathrm{CO}$

$$
=\frac{1}{K V} \sum_{J} B_{J}^{2}-C_{0}
$$

b) SQTrat.ajust. $=\hat{\tau}^{\prime} Q=Q^{\prime} M^{-1} Q \quad(452,5)$

$$
\begin{aligned}
& =\hat{\tau^{\prime}} Q=\left[\begin{array}{l:l|l}
\hat{\tau}_{c}^{\prime} & \hat{\tau}_{r}^{\prime} & \mid Q_{C} \\
\hdashline & Q_{r}
\end{array} \mid\right. \\
& =\hat{\tau}_{c}^{\prime} Q_{C}+\hat{\tau}_{r}^{\prime} Q_{r} \quad(452.6)
\end{aligned}
$$

ou ainda

$$
\text { SQTrat ajust }=\sum_{i} \hat{t}_{i}{ }_{i}+\sum_{i^{\prime} j^{t}} \hat{i}^{\prime} j^{2}{ }^{\prime} j
$$

substituindo $\hat{t}_{i} e \hat{t}_{i^{\prime} j}$ pelos seus valores dados em (44.12) e (44.13) respectivamente, tem-se

$$
\begin{aligned}
& \text { SQtrat aj }=\sum_{i}\left(\frac{1}{b v} Q_{i}\right) Q_{i}+\sum_{i^{j}}\left(\frac{1}{v} Q_{i^{\prime} j}+\frac{1}{c v} \sum_{i^{\prime}} 2_{i^{\prime} j}+\frac{1}{b c v} \sum_{i} Q_{i} Q_{i^{\prime} j}\right. \\
& =\frac{1}{b v} \sum_{i} Q_{i}^{2}+\frac{1}{v} \sum_{i^{\prime} j} Q_{i^{\prime} j}^{2}+\frac{1}{b c v} \sum_{i} Q_{i} \sum_{i^{\prime} j^{\prime}} 2_{i^{\prime} j}+\frac{1}{c v} \sum_{i^{\prime} j}\left(\sum_{i^{\prime}} Q_{i} j^{\prime} Q_{i^{\prime} j}\right.
\end{aligned}
$$


mas

$$
\begin{aligned}
& \sum_{i} Q_{i}=-\sum_{i^{\prime} j} Q_{i} j \mathrm{e} \\
& \sum_{i^{\prime} j}\left(\sum_{i^{\prime}} 2_{i^{\prime} j}{ }^{\prime} 2_{i^{\prime} j}=\sum_{j}\left(\sum_{i^{\prime}} 2_{i^{\prime} j}\right)^{2}\right.
\end{aligned}
$$

$\therefore$ SQtrat aj= $\frac{1}{b v} \sum_{i} Q_{i}^{2}+\frac{1}{v} \sum_{i^{\prime} j^{\prime}} Q_{i^{\prime} j}^{2}-\frac{1}{b c v}\left(\sum_{i} Q_{i}\right)^{2}+\frac{1}{c v} \sum_{j}\left(\sum_{i^{\prime}} Q^{\prime} j\right)^{2}(452,8)$

outra forma de apresentar a sQtrat aj, é atravēs dos totais, resultando.

SQtrat aj $=\frac{1}{b v} \sum_{i} T_{i}^{2}+\frac{1}{v} \sum_{i} T_{j}^{2} T^{\prime} j-\frac{1}{b c v}\left(\sum_{i} T_{i}\right)^{2}+\frac{1}{c v} \sum_{j}\left(\sum_{i}^{*} T_{i}\right)^{2} / j-$

$$
-\frac{1}{K v} \underset{j}{\Sigma}\left(B_{j}\right)^{2}
$$

assim SQP (a) = Co+ SQtrat aj + SQBlocos (452.9)

e a soma de quadrados de Resíduo(a) é dada por

SQRes $(a)=$ SQParcelas - SQtrat aj-SQBlocos (452.10)

e para a soma de quadrado de parâmetro (b), tem-se

$\operatorname{SQP}(\dot{b})=\hat{a^{\prime}} A+\hat{t a} \hat{a}^{\prime} T A+\hat{b a^{\prime} B A}$ 
fazendo

a) $\mathrm{SQ}$ anos $=\hat{a}^{\prime} \mathrm{A}$

substituindo $\hat{a}$, de (44.16), tem-se

$S Q$ anos $=\left[A^{\prime} U^{-1}-\hat{m} a U^{-1}\right] A$

$$
=A^{\prime} U^{-1} A-m a U^{-1} A
$$

$\operatorname{mas} d U^{-1} A=G$

$\therefore S Q$ anos $=A^{\prime} \cdot U^{-1} \not A-\hat{m G}=A \cdot U^{-1} A-C o \quad(452.12)$

ou ainda

SQ anos $=\frac{1}{b K} \sum_{V} A_{V}^{2}-\operatorname{Co}(452.13)$

b) . Sqtxa $=\hat{t a} \cdot \mathrm{TA}$

substituindo tá', de $(44.24)$, tem-se

$$
\begin{aligned}
\text { SQtxa } & =\mathrm{TA}^{\prime}\left(\mathrm{L}^{-1}+\frac{1}{\mathrm{c}} \mathrm{L}^{-1} \mathrm{BB}^{\prime} L^{-1}\right) \mathrm{TA}-\frac{1}{\mathrm{C}} \mathrm{BA}^{\prime} \mathrm{B}^{\prime} \mathrm{L}^{-1} \mathrm{TA}- \\
& -\mathrm{A}^{\prime} \mathrm{U}^{-1} \mathrm{HL} \mathrm{L}^{-1} \mathrm{TA}-\mathrm{Q}^{\prime} \mathrm{M}^{-1} \mathrm{SL} \mathrm{L}^{-1} \mathrm{TA}
\end{aligned}
$$

$\operatorname{mas} Q^{\prime} M^{-I^{\prime}}=\hat{\tau^{\prime}}$ e $S L^{-1} \mathrm{TA}=\mathrm{T} ; A^{\prime} U^{-1} \mathrm{HL}^{-1} \mathrm{TA}=\frac{1}{b K} A^{\prime} A$

$$
\begin{aligned}
& \text { SQtxa }=\mathrm{TA}^{\prime}\left[L^{-1}+\frac{1}{\mathrm{c}} \mathrm{L}^{-1} \mathrm{BB}^{\prime} L^{-1}\right] \mathrm{TA}-\frac{1}{\mathrm{C}} \mathrm{BA}^{\prime} \mathrm{B}^{\prime} L^{-1} \mathrm{TA}-\frac{1}{b K} \mathrm{~A}^{\prime} \mathrm{A}-\hat{\tau}^{\prime} \mathrm{T} \\
& \text { setxa }=\mathrm{TA}^{\prime}\left[\mathrm{L}^{-1}+\frac{1}{\mathrm{c}} \mathrm{L}^{-1} \mathrm{BB}^{\prime} \mathrm{L}^{-1}\right] \mathrm{TA}-\frac{1}{\mathrm{c}} \mathrm{BA}^{\prime} \mathrm{B}^{\prime} \mathrm{L}^{-1} \mathrm{TA}-\mathrm{SQa}- \\
& \text { - se trat } \text { usual }^{- \text {Co }}
\end{aligned}
$$


onde

$$
\begin{aligned}
& \mathrm{TA}^{\prime}\left[\mathrm{L}^{-1}+\frac{1}{c} L^{-1} \mathrm{BB}^{\prime} L^{-1}\right] \mathrm{TA}=\frac{1}{b} \sum_{i v}\left(T A_{i v^{\prime}}\right)^{2}+\sum_{i^{\prime} j v}\left(T A_{i^{\prime} j u}\right)^{2}+ \\
& +\frac{1}{c b} \sum_{v} A_{v} \sum_{i} T A_{i v}+\frac{1}{c b} \sum_{v}\left(\sum_{i} T A_{i v} \mid 1 \sum_{i j} T A_{i j} j\right)+ \\
& +\frac{1}{c} \sum_{j v}\left(\sum_{i} T_{i} A_{i v}\right)^{2} \\
& \frac{1}{c} B^{\prime} B^{\prime} L^{-1} T A=\frac{1}{b c}\left[\sum_{v} A_{v} \sum_{i} T A_{i v}\right]+\frac{1}{c} \sum_{j v}\left[B A_{j v} \sum_{i}, T A_{i j v}\right] \\
& =\frac{1}{b c}\left[\sum_{v} A_{v} \sum_{i} T A_{i v}\right]+\frac{1}{c} \sum_{j v}\left[\sum_{i} T_{i} T A_{i} j v^{2}+\right. \\
& +\frac{1}{c} \sum_{j v}\left[\left(\sum_{l}^{*} T A_{i v} \| / j \quad \sum_{i} T A_{i}^{\prime} j\right]\right. \\
& \frac{1}{b K} A^{\prime} A-C O=S Q \text { anos } \\
& \hat{\tau}^{\prime} T=S Q \text { trat usual }
\end{aligned}
$$$$
\text { tendo-se assim }
$$

$$
\begin{aligned}
& \text { SQtxa }=\frac{1}{b} \sum_{i v}\left(T A A_{i v}\right)^{2}+\sum_{i^{\prime} j v^{\prime}}\left(T A_{i}^{\prime} j v\right)^{2}+\frac{1}{c b} \sum_{v}\left[\left(\sum_{i} T A_{i v}\right)\left(\sum_{i j} T^{\prime} A_{i^{\prime} j v}\right)\right]- \\
& -\frac{1}{c} \sum_{j v}\left[\left|\sum_{i} * T A_{i v}\right| / j \mid \sum_{i}, T A_{i}^{\prime} j v-\right. \\
& \text { - SQanos- sQtrat usual- Co }
\end{aligned}
$$


c) $\mathrm{SQbxa}=\hat{\mathrm{ba}} \hat{\mathrm{a}}^{\prime} \mathrm{BA}$

substituindo ba, de (44.21), tem-se

sQbxa $=\frac{1}{c} B^{\prime} B A-\hat{b}^{\prime} \psi^{\prime} B A-\frac{1}{c} T^{\prime} I^{-1} B B A$

mas $\psi^{\prime} B A=\mathbf{B}$

SQbxa $=\frac{1}{c} B A^{\prime} B A-\hat{b}^{\prime} B-\frac{1}{c} T^{\prime} L^{-1} B B A$

SQbxa $=\frac{1}{c} \mathrm{BA}^{\prime} \mathrm{BA}-\frac{1}{\mathrm{c}} \mathrm{TA}^{\prime} \mathrm{L}^{-1} \mathrm{BBA}-\mathrm{SQBlocOS}$ * (452.16)

onde

$\frac{1}{c} B A^{\prime} B A=\frac{1}{c} \sum_{j v} B A_{j v}^{2}$

$\frac{1}{c} T^{\prime} L^{-1} B B A=\frac{1}{b c}\left[\sum_{v} A_{v} \sum_{i}^{\sum} T A_{i v}\right]+\frac{1}{c} \sum_{j v}\left(\sum_{i} T A_{i}^{\prime} j v\right)^{2}+$

$+\frac{1}{c} \sum_{j v}\left[\left(\mid \sum_{i}^{*} T A_{i v}\right) / j\left|\sum_{i} T A_{i_{j}^{\prime}}\right|\right.$

SQblocos* $=\hat{b^{\prime}} B=\left[B^{\prime} A^{-I_{B}}-\operatorname{Co-\hat {\tau }} ' T+\hat{\tau^{\prime} Q}\right]=\sum_{j} \hat{b}_{j} \cdot B_{j}$

substituindo o valor de $\hat{b}_{j}$ dado em 44.15

$\sum\left[\frac{1}{c v}\left(\sum_{i}^{*} T_{i} / / j-\frac{1}{b c v} \sum_{i} T_{i}\right] B_{j}=\right.$

$=\frac{1}{c v} \sum_{j}\left(\sum_{i}^{*} T_{i}\right) / \dot{j} \cdot B_{j}-\frac{1}{b c v}\left(\sum_{i} T_{i}\right) \sum_{j} B_{j}=$

$=\frac{1}{C V} \sum_{j}\left(\sum_{i}^{*} T_{i}\right) / j \cdot B_{j}-\sum_{i} T_{i} \frac{G}{b c v}=$

$=\frac{1}{C V} \sum_{j}\left(\sum_{i} * T_{i}\right) / j \cdot B_{j}-m \sum_{i} T_{i}=S Q$ blocos * 
$\therefore$ SQbxa $=\frac{1}{c} \sum_{j v} B A_{j v}^{2}-\frac{1}{b c}\left[\sum_{v} A_{v} \sum_{i}^{\sum} T A_{i v}\right]-\frac{1}{c} \sum_{j v}\left(\sum_{i} T_{i^{\prime}}{ }_{j v}\right)^{2}-$

$$
-\frac{1}{c} \sum_{j v}\left[\left(\sum_{i}^{*} T A_{i v}\right) / j \sum_{i}^{\prime} T A_{i j v}^{\prime}\right]-\operatorname{sQblocos}{ }^{*}
$$

assim

$\mathrm{SQP}(\mathrm{b})=$ SQanos + SQtxa + SQbxa

e para a soma de quadrado de Resíduo (b)

SQRes $(b)=$ SQTotal - SQP(b) - SQParcelas

onde SQTotal $=Y^{\prime} Y-$ Co

$\therefore$ SQRes $(b)=Y^{\prime} Y-C o-S Q a n o s$ - SQtxa - SQbxa - SQParcelas

$$
\ldots(452.18)
$$

$\therefore$ SQRes $(b)=Y^{\prime} Y-C O-S Q a n o s-S Q t x a-S Q b x a-\frac{1}{v} Y^{\prime} \Phi Y+C O$

$$
=Y^{\prime}\left[I-\frac{1}{v} \Phi\right] Y-\text { SQanos -SQtxa- SQbxa ...(452,19) }
$$


4.6. Esperança das Somas de Quadrados

Para o desenvolvimento das esperanças matemáticas das somas de quadrados, as mesmas restrições usadas para a solução do sistema de equações, dadas em 4.2 , são usadas ago ra para os parâmetros.

No desenvolvimento deste capitulo, utilizaremos as mesmas metodologias empregadas por IEMMA (1981).

4.6.1. Média Geral

$$
\begin{aligned}
& \hat{S Q \hat{m}}=\hat{m G}=\frac{G^{2}}{b K v}=\frac{G^{2}}{n} \\
& E(S Q \hat{m})=\frac{1}{n} E\left(G^{2}\right)=\frac{1}{n} E\left(Y^{\prime} X_{0} X_{0}^{\prime} Y\right)= \\
& =\frac{1}{n} E\left[\left(\theta X^{\prime}+\varepsilon^{\prime}\right) X_{0} X_{0}^{\prime}(X \theta+\varepsilon)\right]
\end{aligned}
$$

desenvolvendo os produtos e aplicando-se a esperança matemātica, considerando que

$$
\operatorname{cov}\left(y_{\ell j v^{\prime}} y_{\ell^{\prime}{ }^{\prime} v^{\prime}}\right)=\left\{\begin{array}{c}
\sigma^{2} \text { se } \ell=\ell^{\prime} ; j=j^{\prime} ; u=v^{\prime} \\
0 \sigma^{2} \text { se } \ell=\ell^{\prime} ; j=j^{\prime} ; u \neq v^{\prime} \\
0 \text { em outros casos. }
\end{array}\right.
$$


$E(S Q \hat{m})=\frac{1}{n} \theta^{\prime} X^{\prime} X_{0} X_{0}^{\prime} X \theta+\frac{1}{n} E\left(\varepsilon^{\prime} X_{0} X_{0}^{\prime} \varepsilon\right)$

onde

i) $\frac{1}{n} \theta^{\prime} x^{\prime} x_{0} x_{0}^{\prime} x \theta=\frac{1}{n} \theta^{\prime}\left(\begin{array}{cccccc}n^{2} & n g & n c & \text { nd } & \text { ne } & n f \\ g^{\prime} n & g^{\prime} g & g^{\prime} c & g^{\prime} d & g^{\prime} e & g^{\prime} f \\ c^{\prime} n & c^{\prime} g & c^{\prime} c & c^{\prime} d & c^{\prime} e & c^{\prime} f \\ d^{\prime} n & d^{\prime} g & d^{\prime} c & d^{\prime} d & d^{\prime} e & d^{\prime} f \\ e^{\prime} n & e^{\prime} g & e^{\prime} c & e^{\prime} d & e^{\prime} e & e^{\prime} f \\ f^{\prime} n & f^{\prime} g & f^{\prime} c & f^{\prime} d & f^{\prime} e & f f\end{array}\right) \theta$

efetuando-se o produto e de acordo com as restrições impostas em 4.2, tem-se as seguintes matrizes nulas

$\tau^{\prime} g^{\prime}=b^{\prime} c^{\prime}=a^{\prime} d^{\prime}=t a^{\prime} e^{\prime}=b a^{\prime} f^{\prime}=\phi$

resultando assim

$\frac{1}{n} \theta^{\prime} x^{\prime} x_{0} x^{\prime} x^{\prime} \theta=n m^{2}$

e em conformidade com IEMMA(1981), para o desenvolvimento de

ii) $\frac{1}{n} E\left(\varepsilon^{\prime} X_{0} X_{0}^{\prime} \varepsilon\right)=\frac{1}{n} T R\left(X_{0} X_{0}^{\prime}\right)[1+(u-1) \rho] \sigma^{2}$

onde TR, significa traço da matriz,

sendo $\mathrm{x}_{0} \mathrm{X}_{0}^{\prime}=b K \cup \mathbf{E}(1 \times 1)$ então $\operatorname{TR}\left(\mathrm{X}_{0} \mathrm{X}_{0}^{\prime}\right)=b K v=n$

$\operatorname{assim} \frac{1}{n} E\left(\varepsilon^{\prime} X_{0} X_{0}^{\prime} \varepsilon\right)=[1+(v-1) \rho] \sigma^{2}$ 
susbtituindo $461.2461 .3 \mathrm{em} 461.1$ tem-se

$E(S Q \hat{m})=n m^{2}+[1+(v-1) \rho] \sigma^{2}$

4.6.2. Tratamentos Principais

De 452.5, tem-se

SQtrat aj $=\hat{\tau}^{\prime} Q=Q M^{-1} Q$

Para minimizar os cálculos devido a substituição da matriz $Q=T-N A^{-1} B$, adotou-se 0 uso da matriz que CHAKRABARTI(1962)， PIMENTEL GOMES(1967)，DINIZ(1980)， chamam de matriz w, a qual é definida por IEMMA(1981), como:

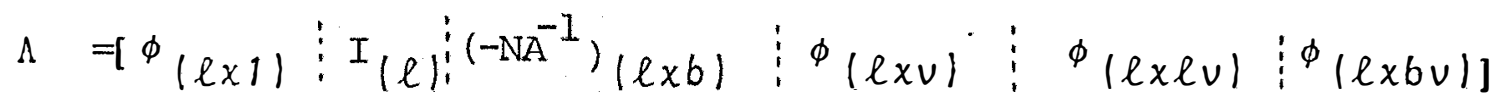

usando a propriedade demonstrada por PIMENTEL GOMES de que $Q=\Lambda X^{\prime} Y$, obtêm-se

$$
\begin{aligned}
\text { Setrat aj } & =Y^{\prime} X \Lambda^{\prime} M^{-I^{\prime}} \Lambda X^{\prime} Y \quad(462.1) \\
& =\left(\theta^{\prime} X^{\prime}+\varepsilon^{\prime}\right) X \Lambda^{\prime} M^{-I^{\prime}} \Lambda X^{\prime}(X \theta+\varepsilon)
\end{aligned}
$$

$E\left(\right.$ SQtrat aj) $=\theta^{\prime} X^{\prime} X \quad \Lambda^{\prime} M^{-I^{\prime}} \Lambda X^{\prime} \quad X \quad \theta+E\left(\varepsilon^{\prime} X \Lambda^{\prime-M^{\prime}} \Lambda X^{\prime} \varepsilon\right) \quad$ (462,2) 
mas $\Lambda \mathrm{X}^{\prime} \mathrm{X} \theta=\boldsymbol{c} \tau=Q \quad$ e $\quad \Lambda \mathrm{X}^{\prime} \mathrm{X}, \Lambda^{\prime}=\boldsymbol{c}$

tem-se assim 462.2, a seguinte estrutura.

$E\left(\right.$ SQtrat aj) $=\tau^{\prime}{C^{\prime}}^{M^{-I^{\prime}}} \boldsymbol{C}_{\tau}+E\left(\varepsilon^{\prime} X \Lambda^{\prime} \boldsymbol{M}^{-l^{\prime}} \Lambda X^{\prime} \varepsilon\right)$

$\operatorname{mas} \mathbf{C}^{\prime} \mathbf{M}^{-1} \mathbf{C}=\mathbf{C} \quad$ e $\quad \boldsymbol{C} \tau=Q$

E'sQtrat $a j)=\tau^{\prime} Q+E\left(\varepsilon^{\prime} X \Lambda^{\prime} M^{-I^{\prime}} \Lambda X^{\prime} \varepsilon\right) \quad(4623)$

no desenvolvimento do termo $E\left(\varepsilon^{\prime} X \Lambda^{\prime} M^{-1} \Lambda X^{\prime} \varepsilon\right)$, utilizando o método apresentado por IEMMA(1981), define-se

$$
\pi_{0}=X \Lambda^{\prime} M^{-1} \Lambda X^{\prime}
$$

$E\left(\varepsilon^{\prime} X \Lambda^{\prime} M^{-1^{\prime}} \Lambda X^{\prime} \varepsilon\right)=E\left(\varepsilon^{\prime} \pi_{0} \varepsilon\right)=\operatorname{TR}\left(\pi_{0}\right)[1+(v-1) \rho] \sigma^{2}$

onde partindo-se a matriz $\pi_{0}$ em $\pi_{1}=X \Lambda^{\prime}$ e $\pi_{2}=M^{-I^{\prime}} \Lambda X^{\prime}$

e usando-se a propriedade de que se existem os produtos $\pi_{1} \pi_{2}$ e $\pi_{2} \quad \pi_{1}$ então $\operatorname{TR}\left(\pi_{1} \pi_{2}\right)=\operatorname{TR}\left(\pi_{2} \pi_{1}\right)$, assim $\operatorname{TR}\left(\pi_{0}\right)=\operatorname{TR}\left(\pi_{2} \pi_{1}\right)=\operatorname{TR}\left(M^{-I^{\prime}} \Lambda X^{\prime} X \Lambda^{\prime}\right)=\operatorname{TR}\left(M^{-1} \mathbf{C}\right)$ mas $\mathbf{M}^{-1} \mathbf{C}$ è idempotentes então 
$\operatorname{TR}\left(M^{-I^{\prime}} \mathbf{C}\right)^{-}=\ell\left(M^{-l^{\prime}} \mathbf{C}\right)$, sendo $\&$ a caracterîstica da matriz. Tendo-se em conta que $\mathbf{c}$ è singular de caracterís tica $l-1=c+z-1$, então como $M^{-1}$ ' $\ddot{e}$ nã̃o sìnguiar $\circ$ produto $\mathbf{M}^{-1} \mathbf{C}$, preserva a característica de $\mathbf{C}$, logo

$\operatorname{TR}\left(M^{-1} \mathbf{C}\right)=\ell(\mathbf{c})=\ell-1$

obtendo-se assim para 462.4,

$E\left(\varepsilon^{\prime} X \Lambda^{\prime} M^{-I^{\prime}} \Lambda X^{\prime} \varepsilon\right)=(\ell-1)[1+(v-1) \rho] \sigma^{2}$

substituindo 462.5 em 462.3 , tem-se

$E\left(\right.$ SQtrat aj) $=\tau^{\prime} Q+(l-1)[1+(v-1) 0] \sigma^{2}$

Da caracteristica de $\mathbf{c}$, obtém-se o número de graus de liberdade para os tratamentos principais: desse modo

$E($ QMtrat $a j)=[1+(v-1) \varepsilon] \sigma^{2}+\frac{\tau^{\prime} Q}{\ell}-1$ $(462.7)$ 


\subsubsection{Blocos}

De 452,4

$$
\begin{aligned}
& S Q b=B^{\prime} A^{-1} B-C_{0} \\
& =Y^{\prime} X_{3} A^{-1} X_{3}^{\prime} Y-C_{0} \\
& E(S Q b)=E\left[\left(\theta^{\prime} X^{\prime}+\varepsilon^{\prime}\right) X_{3} A^{-1} X_{3}^{\prime}(X \theta+\varepsilon)\right]-E\left(C_{0}\right) \\
& =\theta^{\prime} X^{\prime} X_{3} A^{-1} X_{3}^{\prime} X \theta+E\left(\varepsilon^{\prime} X_{3} A^{-1} X_{3}^{\prime} \varepsilon\right)-E\left(C_{0}\right)
\end{aligned}
$$

desenvolvendo por partes tem-se

i) $\theta^{\prime} X^{\prime} X_{3} A^{-1} X_{3}^{\prime} X \theta=\theta^{\prime}\left(\begin{array}{cccccc}c A^{-1} C^{\prime} & c A^{-1} N^{\prime} & c & c A^{-1} K & c A^{-1} V & c A^{-1} F \\ N A^{-1} C^{\prime} & N A^{-1} N^{\prime} & N & N A^{-1} K & N A^{-1} V & N A^{-1} F \\ C^{\prime} & N^{\prime} & A & K & V & F \\ K^{\prime} A^{-1} C^{\prime} & K A^{-1} N^{\prime} & K^{\prime} & K^{\prime} A^{-1} K & K^{\prime} A^{-1} V & K^{\prime} A^{-1} F \\ V^{\prime} A^{-1} C^{\prime} & V^{\prime} A^{-1} N^{\prime} & V & V^{\prime} A^{-1} K & V^{\prime} A^{-1} V & V^{\prime} A^{-1} F \\ F^{\prime} A^{-1} C^{\prime} & F^{\prime} A^{-1} N^{\prime} & F^{\prime} & F^{\prime} A^{-1} K & F^{\prime} A^{-1} V & F^{\prime} A^{-1} F\end{array}\right) \theta$

efetuando-se os produtos, e de acordo com as restrições impos tas, tem-se as seguintes matrizes nulas.

$\tau^{\prime} N A^{-1} C^{\prime} ; b^{\prime} c^{\prime} ; a^{\prime} K^{\prime} A^{-1} C^{\prime} ; t a^{\prime} V^{\prime} A^{-1} C^{\prime} ; b a^{\prime} F^{\prime} A^{-1} c^{\prime} ; c A^{-1} N^{\prime} \tau$; $a^{\prime} K^{\prime} A^{-1} N^{\prime} ; t a^{\prime} V^{\prime} A^{-1} N^{\prime} ; b a^{\prime} F^{\prime} A^{-1} N^{\prime} ; a^{\prime} K^{\prime} ; t a^{\prime} V^{\prime} ; b a^{\prime} F^{\prime}$ 
assim

$\theta^{\prime} X^{\prime} X_{3} A^{-1} X_{3}^{\prime} X \theta=n m^{2}+\tau^{\prime} N A^{-1} N^{\prime} \tau+2 b^{\prime} N^{\prime} \tau+b^{\prime} A b$

mas $\mathrm{R}-\boldsymbol{C}=\mathrm{NA}^{-1} \mathrm{~N}^{\prime}$;

$\therefore \theta^{\prime} X^{\prime} X_{3} A^{-1} X_{3}^{\prime} X \theta=n m^{2}+\tau^{\prime} R^{\prime} \tau-\tau^{\prime} c \tau+2 b^{\prime} N^{\prime} \tau+b^{\prime} A b$

ii) $E\left(\varepsilon^{\prime} \mathrm{X}_{3} \mathrm{~A}^{-1} \mathrm{X}_{3}^{\prime} \varepsilon\right)$

define-se $\pi_{3}=\mathrm{X}_{3} \mathrm{~A}^{-1} \mathrm{X}_{3}^{\prime} ; \pi_{4}=\mathrm{x}_{3}$ e $\pi_{5}=\mathrm{A}^{-1} \mathrm{X}_{3}^{\prime}$, tendo-se

$E\left(\varepsilon^{\prime} X_{3} A^{-1} X_{3}^{\prime} \varepsilon\right)=E\left(\varepsilon^{\prime} \pi_{3} \varepsilon\right)=\operatorname{TR}\left(\pi_{3}\right)[1+(u-1) \rho] \sigma^{2}$

onde $\operatorname{TR}\left(\pi_{3}\right)=\operatorname{TR}\left(\pi_{4} \cdot \pi_{5}\right)=\operatorname{TR}\left(\pi_{5} \cdot \pi_{4}\right)=$

$$
=\operatorname{TR}\left(A^{-\lambda_{A}}\right)=\operatorname{TR}(I(b))=b
$$

$\therefore \quad E\left(\varepsilon^{\prime} X_{3} A^{-1} X_{3}^{1} \varepsilon\right)=b[1+(v-1) 0.] \sigma^{2}$

iii) $E(C o)=E(S Q \hat{m})=n m^{2}+[1+(v-I) \rho] \sigma^{2}$ de $(461.4)$

susbtituindo 461,$4 ; 463,2 ; 463,3$ em 463.1 , tem-se

$E(S Q b)=(b-1)[1+(v-1) \rho] \sigma^{2}+\tau^{\prime} R \tau-\tau^{\prime} \mathbf{C} \tau+2 b^{\prime} N^{\prime} \tau+b^{\prime} A b$

como $\boldsymbol{C}_{\tau}=Q$ 
$E(S Q b)=(b-1)[1+(u-1) \rho] \sigma^{2}+\tau^{\prime} R \tau-\tau^{\prime} Q+2 b^{\prime} N^{\prime} \tau+b^{\prime} A b$

ou ainda

$E(S Q b)=(b-1)[1+(v-1) p] \sigma^{2}+v b \sum_{i} t_{i}^{2}+v \sum_{i j} t_{i}^{2} j+$

$$
+2 v \sum_{j} b_{j} \sum_{i} t_{i^{\prime} j}+K v \underset{j}{\sum} b_{j}^{2}-\tau^{\prime} Q
$$

e $E(Q M b)=[1+(U-1) \rho] \sigma^{2}+\frac{1}{b-1}\left\{\tau^{\prime} R \tau-\tau^{\prime} Q+2 b^{\prime} N^{\prime} \tau+b^{\prime} A b\right\}$

$\ldots(4636)$

4.6.4. Soma de Quadrados de Parcelas

$\mathrm{SQParC}=\frac{1}{v} \mathrm{Y}^{\prime} \Phi \mathrm{Y}-\mathrm{CO}$

$E(\operatorname{SQParC})=\frac{1}{v} E\left[\left(\theta^{\prime} X^{\prime}+\varepsilon^{\prime}\right) \Phi(X \theta+\varepsilon)\right]-E(C O)$

$$
=\frac{1}{v}\left(\theta^{\prime} X^{\prime} \Phi X \theta\right)+\frac{1}{v} E\left(\varepsilon^{\prime} \Phi \varepsilon\right)-E(C O)
$$


resolvendo por pärtes tem-se

i) $\frac{1}{v} \theta^{\prime} \mathrm{X}^{\prime} \Phi \mathrm{X} \theta=\frac{1}{v} \theta^{\prime}\left[\begin{array}{llllll}v \mathrm{n} & v g & v \mathrm{C} & v \mathrm{~d} & v e & v f \\ v g^{\prime} & v \mathrm{R} & v \mathrm{~N} & v \mathrm{P} & v S & v \mathrm{D} \\ v \mathrm{C}^{\prime} & v \mathrm{~N}^{\prime} & v A & v \mathrm{~K} & v \mathrm{~V} & v \mathrm{~F} \\ v \mathrm{~d}^{\prime} & v \mathrm{P}^{\prime} & v \mathrm{~K}^{\prime} & \mathrm{U}^{*} & \mathrm{H}^{*} & \mathrm{G}^{*} \\ v \mathrm{e}^{\prime} & v S^{\prime} & v V^{\prime} & \mathrm{H}^{* \prime} & \mathrm{L}^{*} & \mathrm{~B}^{*} \\ v \mathrm{f}^{\prime} & v \mathrm{D}^{\prime} & v \mathrm{~F}^{\prime} & \mathrm{G}^{* \prime} & \mathrm{B}^{* \prime} & \mathrm{W}^{*}\end{array}\right] \theta$

sendo, $v$ o nümero de tratamentos secundārios

$U^{*}=K E_{(u \times v)} ; \quad K$ número de parcelas por bloco

$H^{*}=\left[H_{0}^{*}: H_{1}^{*}\right] ; H_{0}^{*}=b \mathbf{E}_{(v x c u)} ; b=$ números de blocos.

$$
H_{I}^{*}=\mathbf{E}(v \times \cup z)
$$

$$
\begin{aligned}
& G^{*}=K E_{(v \times b u)} \\
& L_{0}^{*}=\text { matriz diagonal de submatrizes } \\
& L^{*}=\left|\begin{array}{c:r}
L_{0}^{*} & L_{1}^{*} \\
\hdashline L_{1}^{* 1} & L_{2}^{*}
\end{array}\right|_{(\ell v \times \ell v)}: L_{1}^{E_{1}^{*}=\phi_{(c \vee c) ~}{ }^{d}} \\
& L_{2}^{*}=\text { matriz de submatrizes } I_{(z)} \text { de } \\
& \text { dimensão } v \bar{z} x \cup z \text {. }
\end{aligned}
$$




$$
\left.B^{*}=\left(\begin{array}{ccc}
B_{0}^{*} & \cdots & B_{0}^{*} \\
B_{1}^{*} & \cdots & B_{1}^{*} \\
B_{1}^{*} & \cdots & B_{1}^{*} \\
\cdots & \cdots & \cdots \\
B_{1}^{*} & \cdots & B_{1}^{*}
\end{array}\right)_{(\ell v} x \text { bv }\right)
$$$$
\begin{aligned}
& B_{0}^{*}=E_{(c v} \\
& B_{1}^{*}=\left[\begin{array}{cccc}
1 & 0 & \ldots & 0 \\
1 & 0 & \ldots & 0 \\
\vdots & \vdots & & \vdots \\
1 & 0 & \ldots & 0 \\
0 & 1 & \ldots & 0 \\
0 & 1 & \ldots & 0 \\
\cdot & \ddots & \ldots & \cdot \\
0 & 1 & \ldots & 0 \\
\cdot & \cdots & \ldots & \cdot \\
0 & 0 & \ldots & 1 \\
\cdot & \cdot & \ldots & \cdot \\
0 & 0 & \ldots & 1
\end{array}\right)_{(z \times b)}
\end{aligned}
$$

$W^{*}=$ matriz de submatrizes $K I(b)$, com $K$ número de parcelas por bloco; dim de $W^{*}=b v x$ bv.

assim efetuando-se os produtos e de acordo com as restriçõẽs. têm-se as seguintes matrizes nulas.

$\tau^{\prime} g^{\prime} ; \mathrm{c} \mathrm{b}$; da; e ta; f ba; a' $\mathrm{P}^{\prime}$; ta' $\mathrm{S}^{\prime}$; $\mathrm{ba}^{\prime} \mathrm{D}^{\prime} ; \mathrm{a}^{\prime} \mathrm{K}^{\prime}$; ta' $V^{\prime} ; b a^{\prime} F^{\prime} ; t a^{\prime} H^{\prime} ; b a^{\prime} G^{*} ;$ ta $B^{*} ; a^{\prime} U^{*} ; t a^{\prime} L^{*} ; b a^{\prime} W^{*}$ assim

$$
\begin{aligned}
& \frac{1}{v}\left(\theta^{\prime} X^{\prime} \Phi X \theta\right)=n m^{2}+\tau^{\prime} R \tau+2 b^{\prime} N^{\prime} \tau+b^{\prime} A b \\
& \text { ii) } \frac{1}{v} E\left(\varepsilon^{\prime} \Phi \varepsilon\right)=T R(\Phi)[1+(v-1) \rho] \sigma^{2}
\end{aligned}
$$


onde $\operatorname{TR}(\Phi)=6 K$

$\frac{1}{v} E\left(\varepsilon^{\prime} \Phi \varepsilon\right)=6 K[1+(v-1) \rho] \sigma^{2}$

iii) $E(C o)=n m^{2}+[1+(v-1) \rho] \sigma^{2}$, de $(4614)$

substituindo os valores $(4642) ;(4643) ;(461.4) ;$ em $(4641)$ tem-se

$E(S Q P a r c)=(b K-1)[1+(v-1) \rho] \sigma^{2}+\tau^{\prime} R \tau+2 b^{\prime} N^{\prime} \tau+b^{\prime} A b \quad(464.4)$

na forma algébrica tem-se

$E($ SQParc $)=v b \sum_{i}^{\Sigma} t_{i}^{2}+v \sum_{i^{\prime} j^{\prime}} t_{i^{\prime} j}^{2}+2 v \sum_{j} b_{j} \sum_{i^{\prime}} t_{i^{\prime} j}+K v \underset{j}{\Sigma} b_{j}^{2}+$ $+(6 K-1)[1+(v-1) \rho] \sigma^{2}$

e $E(Q M P a r c)=[1+(U-1) \rho] \sigma^{2}+\frac{1}{b K-1}\left[\tau^{\prime} R \tau+2 b^{\prime} N^{\prime} \tau+b^{\prime} A b\right]$ ... $(464.6)$

4.6.5. Residuo (a) De 452.10 tem-se

SQRes $(a)=$ SQParC - SQTrat aj - SQBlocos.

$E($ SQRes $(a))=E($ SQParC $)-E($ SQtrat aj) $-E($ SQBlocos $)$ 
substituindo os valores (462.7); (463.4); (464.4); em (465.1)

$E(\operatorname{SQReS}(a))=[b(K-1)-(\ell-1)][1+(v-1) \rho] \sigma^{2}$

$\therefore E[\operatorname{QMRes}(a)]=[1+(u-1) \rho] \sigma^{2}$

\subsubsection{Tratamentos Secundários}

$S Q a=A^{\prime} U^{-1} A-C o$ de $(452,12)$

$\mathrm{SQa}=\mathrm{Y}^{\prime} \mathrm{X}_{4} \mathrm{U}^{-1} \mathrm{X}_{4}^{\prime} \mathrm{Y}-\mathrm{Co}$

$E(S Q a)=E\left[\left(\theta^{\prime} X^{\prime}+\varepsilon^{\prime}\right) X_{4} U^{-1} X_{4}^{\prime}(X \theta+\varepsilon)\right]-E(C o)$

$E(S Q a)=\theta^{\prime} X^{\prime} X_{4} U^{-1} X_{4}^{\prime} X \theta+E\left(\varepsilon^{\prime} X_{4} U^{-1} X_{4}^{1} \varepsilon\right)-E(C o) \quad(466,1)$

resolvendo por partes tem-se

i) $\theta^{\prime} X^{\prime} X_{4} U^{-1} X_{4}^{\prime} X \theta=\theta^{\prime}\left(\begin{array}{cccccc}d U^{-1} d^{\prime} & d U^{-1} P^{\prime} & d U^{-1} K^{\prime} & d & d U^{-1} H & d U^{-1} G \\ P U^{-1} d^{\prime} & P U^{-1} P^{\prime} & P U^{-1} K^{\prime} & P & P U^{-1} H & P U^{-1} G \\ K U^{-1} d^{\prime} & K U^{-1} P^{\prime} & K U^{-1} K^{\prime} & K & K U^{-1} H & K U^{-1} G \\ d^{\prime} & P^{\prime} & K^{\prime} & U & H & G \\ H^{\prime} U^{-1} d^{\prime} & H^{\prime} U^{-1} P^{\prime} & H^{\prime} U^{-1} K^{\prime} & H^{\prime} & H^{\prime} U^{-1} H & H^{\prime} U^{-1} G \\ G^{-1} U^{-1} d^{\prime} & G^{\prime} U^{-1} P^{\prime} & G U^{-1} K^{\prime} & G^{\prime} & G^{\prime} U^{-1} H & G^{\prime} U^{-1}\end{array}\right) \theta$ 
efetuando-se os produtos e de acordo com as restrições impostas, temos as seguintes matrizes nulas

$\tau^{\prime} P ; b^{\prime} K ; a^{\prime} d^{\prime} ; t a^{\prime} H^{\prime} ; b^{\prime} G^{\prime}$

$e d^{\prime} u^{-1} d^{\prime}=n$

tendo-se então

$\theta^{\prime} X^{\prime} X_{4} U^{-1} X^{\prime} X \theta=n m^{2}+a^{\prime} U a$

ii) $E\left(\varepsilon^{\prime} X_{4} U^{-1} X_{4}^{\prime} \varepsilon\right)$

fazendo $\pi_{6} \doteq \mathrm{x}_{4} \mathrm{U}^{-1} \mathrm{X}_{4}^{\prime} ; \pi_{7}=\mathrm{x}_{4}$ e $\pi_{8}=\mathrm{U}^{-1} \mathrm{X}_{4}^{\prime}$, tem-se

$\operatorname{TR}\left(\pi_{6}\right)=\operatorname{TR}\left(\pi_{7} \pi_{8}\right)=\operatorname{TR}\left(\pi_{8} \pi_{7}\right)=\operatorname{TR}\left(U^{-1} U\right)=\operatorname{TR}(I(U))=v$

no entanto, para a correção 4.6.1., para tratamentos principais $4.6 .2 .$, e para blocos 4.6 .3 ., foram obtidas

$E\left(\varepsilon^{\prime} \pi^{*} \varepsilon\right)=[1+(v-1) \rho] \sigma^{2}+f(\mathbf{P})$

sendo $\pi^{*}$, uma das matrizes ali definidas, e $f(\mathbf{P})$ uma função de parâmetros envolvidos, segundo IEMMA(1981), o resultado obtido para o termo que envolve a variância é diferente; e que é justificado pela estrutura da matriz $x_{4}$, ou seja: 
$\varepsilon^{\prime} x_{4}=\left[\sum_{i j} e_{i j 1} \sum_{i j} \quad e_{i j 2} \cdots \sum_{i j} e_{i j u}\right]$

$\therefore \varepsilon^{\prime} \mathrm{X}_{4} \mathrm{X}_{4}^{\prime} \varepsilon=\left[\sum_{i j} e_{i j 1}^{2}+\sum_{i j} e_{i j 2}^{2}+\ldots+\sum_{i j} e_{i j v}^{2}+\right.$ duplos produtos. $]$

$\therefore E\left(\varepsilon^{\prime} \mathrm{X}_{4} \mathrm{X}_{4}^{\prime} \varepsilon\right)=b K V \quad \sigma^{2}$

portanto

$E\left(\varepsilon^{\prime} X_{4} U^{-1} X_{4}^{\prime} \varepsilon\right)=\frac{1}{b K} E\left(\varepsilon^{\prime} X_{4}^{I}(v)_{4}^{\prime} \varepsilon\right)=$

$$
=\frac{1}{b K} E\left(\varepsilon^{\prime} \mathrm{X}_{4} \mathrm{X}_{4}^{\prime} \varepsilon\right)=\cup \sigma^{2} \quad(466.3)
$$

substituindo $(466.2) ;(466,3) ;(461.4) \mathrm{em}(466.1)$ tem-se

$E(S Q a)=a^{\prime} U a+[(u-1)(1-p)] \quad \sigma^{2} \quad(466.4)$

e na forma algêbrica tem-se

$E($ SQa $)=6 K \sum_{v} a_{v}^{2}+(v-7)(1-p) \sigma^{2}$

$\therefore E(Q M a)=(1-\rho) \sigma^{2}+\frac{a^{\prime} U a}{(U-1)}$ 
.64 .

4.6.7. Interação txa

De (452.14) temos

SQtxa $=\mathrm{TA}^{\prime} \mathrm{L}^{-1} \mathrm{TA}-\frac{1}{\mathrm{c}} \mathrm{BA}^{\prime} \mathrm{B}^{\prime} \mathrm{L}^{-1} \mathrm{TA}+\frac{1}{\mathrm{c}} \mathrm{TA}^{\prime} \mathrm{L}^{-1} \mathrm{BB}^{\prime} \mathrm{L}^{-1} \mathrm{TA}-$

- SQa - sQtrat usual $^{-\mathrm{Co}}$

$E($ SQtxa $)=E\left[\mathrm{TA}^{\prime} \mathrm{L}^{-1} \mathrm{TA}-\frac{1}{\mathrm{C}} \mathrm{BA}^{\prime} \mathrm{B}^{\prime} \mathrm{L}^{-1} \mathrm{TA}+\frac{1}{\mathrm{C}} \mathrm{TA}^{\prime} \mathrm{L}^{-1} \mathrm{BB}^{\prime} \mathrm{L}^{-1} \mathrm{TA}-\right.$

$$
\text { - sQa - sQtrat } \text { usual }^{-\mathrm{Co}} \text { (467.1) }
$$

aplicando-se a esperança e resolvendo por partes tem-se

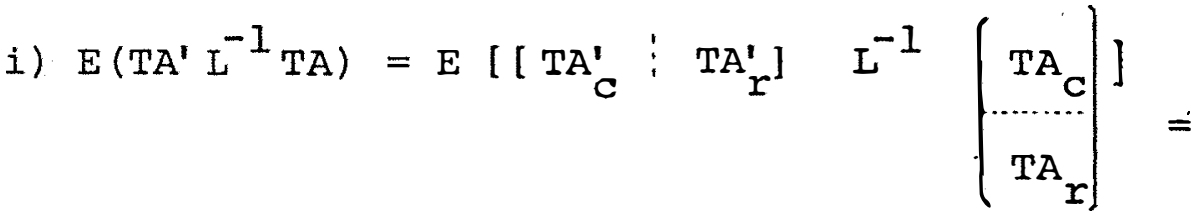

$$
\begin{aligned}
& =E\left[Y^{\prime}\left[\begin{array}{l:l}
X_{5} & \left.X_{6}\right] L^{-1} \\
\hdashline X_{5}^{\prime} \\
X_{6}^{\prime}
\end{array}\right]^{Y}=\right. \\
& =E\left[\left[\theta^{\prime} X^{\prime}+\varepsilon^{\prime}\right]\left[X_{5}: X_{6}\right] L^{-1}\left(\begin{array}{c}
X_{5}^{\prime} \\
X_{6}^{\prime}
\end{array}\right][X \theta+\varepsilon]\right]
\end{aligned}
$$


desenvolvendo o produto e aplicando o conceito de esperança matemática tem-se:

$$
\begin{aligned}
E\left(T^{\prime} L^{-1} T A\right) & =\theta^{\prime} X^{\prime} \quad\left[X_{5}: X_{6}\right] L^{-1}\left(\begin{array}{c}
X_{5}^{\prime} \\
\hdashline X_{6}^{\prime}
\end{array}\right){ }^{\prime} \theta+ \\
& +E\left[\varepsilon^{\prime}\left[X_{5} ! X_{6}\right] L^{-1}\left(\begin{array}{c}
X_{5}^{\prime} \\
X_{6}^{\prime}
\end{array}\right) \varepsilon\right]
\end{aligned}
$$

desenvolvendo por partes tem-se

a) $\theta^{\prime} X^{\prime}\left[x_{5}: x_{6}\right] L^{-1}\left[\begin{array}{c}x_{5}^{\prime} \\ \hdashline x_{6}\end{array}\right] \quad X \theta=$

$=\theta^{\prime}\left(\begin{array}{cccccc}e L^{-1} e^{\prime} & e L^{-1} S^{\prime} & e L^{-1} V^{\prime} & e L^{-1} H^{\prime} & e & e L^{-1} B \\ S L^{-1} e^{\prime} & S L^{-1} S^{\prime} & S L^{-1} V^{\prime} & S L^{-1} H^{\prime} & S & S L^{-1} B \\ V L^{-1} e^{\prime} & V L^{-1} S^{\prime} & V L^{-1} V^{\prime} & V L^{-1} H^{\prime} & V & V L^{-1} B \\ H L^{-1} e^{\prime} & H L^{-1} S^{\prime} & H L^{-1} V^{\prime} & H L^{-1} H^{\prime} & H & H L^{-1} B \\ e^{\prime} & S^{\prime} & V^{\prime} & H^{\prime} & L & B \\ B^{\prime} L^{-1} e^{\prime} & B^{\prime} L^{-1} S^{\prime} & B^{\prime} L^{-1} V^{\prime} & B^{\prime} L^{-1} H^{\prime} & B^{\prime} & B^{\prime} L^{-1} B\end{array}\right] \theta$ 
mas
e $L^{-1} e^{\prime}=n \quad S L^{-1} S^{\prime}=R$
$\mathrm{H} \mathrm{I} \mathrm{L}^{-1} \mathrm{H}^{\prime}=\mathrm{U}$
S $L^{-l} e^{\prime}=g^{\prime}$
$V L^{-1} S^{\prime}=N^{\prime} \quad B^{\prime} L^{-1} H^{\prime}=G^{\prime}$
$\mathrm{V} \mathrm{L}^{-1} \mathrm{e}^{\prime}=\mathrm{c}^{\prime}$
$\mathrm{H} \mathrm{I^{-1 }} \mathrm{S}^{\prime}=\mathrm{P}^{\prime}$
$V L^{-1} V^{\prime}=\psi_{1}^{*} \quad H \quad I^{-1} e^{\prime}=d^{\prime}$
$B^{\prime} L^{-1} S^{\prime}=D^{\prime} \quad B^{\prime} L^{-1} V^{\prime}=\psi_{2}^{*}$
$B^{\prime} L^{-1} e^{\prime}=E^{\prime}$
$\mathrm{H} \mathrm{I}^{-1} \mathrm{~V}^{\prime}=\mathrm{K}^{\prime}$
$\mathrm{B}^{\prime} \mathrm{L}^{-1} \mathrm{~B}=\psi_{3}^{*}$

onde

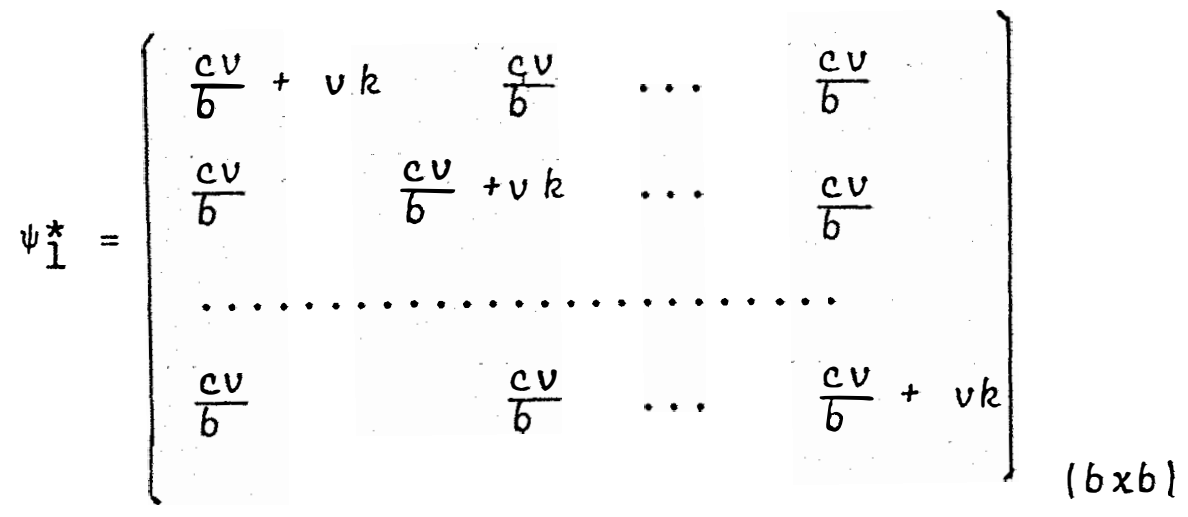

$$
\psi_{2}^{*}=\left(\begin{array}{ccc}
\frac{1}{v} & \psi_{1}^{*} \\
\ldots & \ldots \\
\cdots & \ldots \\
\cdots & \ldots & \psi_{1}^{*}
\end{array}\right)_{(b v \times b)}
$$


$\psi_{3}^{*}=$ matriz diagonal de submatrizes $\frac{1}{v} \psi_{1}^{*} ;$ de dim $(b v x b u)$ assim (467.3) pode ser reescrita da seguinte forma

$$
\theta^{\prime}\left(\begin{array}{llllll}
\mathrm{n} & \mathrm{g} & \mathrm{C} & \mathrm{d} & \mathrm{e} & \mathrm{f} \\
\mathrm{g}^{\prime} & \mathrm{R} & \mathrm{N} & \mathrm{P} & \mathrm{S} & \mathrm{D} \\
\mathrm{C}^{\prime} & \mathrm{N}^{\prime} & \psi_{1}^{*} & \mathrm{~K} & \mathrm{~V} & \psi_{2}^{*^{\prime}} \\
\mathrm{C}^{\prime} & \mathrm{P}^{\prime} & \mathrm{K}^{\prime} & \mathrm{U} & \mathrm{H} & \mathrm{G} \\
\mathrm{e}^{\prime} & \mathrm{S}^{\prime} & \mathrm{V}^{\prime} & \mathrm{H}^{\prime} & \mathrm{I} & \mathrm{B} \\
\mathrm{f}^{\prime} & \mathrm{D}^{\prime} & \psi_{2}^{*} & \mathrm{G}^{\prime} & \mathrm{B}^{\prime} & \psi_{3}^{*}
\end{array}\right] \theta
$$

efetuando-se os produtos, e de acordo com as restrições têm-se as seguintes matrizes nulas.

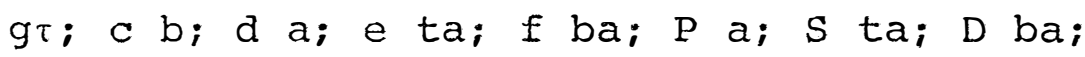

$\mathrm{Ka}$; V ta; ba' ${ }_{2}^{*} ; \mathrm{H}$ ta; G ba;

e as seguintes matrizes não nulas

$\mathrm{mn} m=\mathrm{n} \mathrm{m}^{2}$

$\tau^{\prime} R \tau=v\left[6 \sum_{i} t_{i}^{2}+\sum_{i j} t_{i j}^{2}\right]$

$b^{\prime} N^{\prime} \tau=v \sum_{j} b_{j} \sum_{i} t_{i} i^{\prime}$

$b^{\prime} \psi_{1}^{*} b=v k b^{\prime} b=v k \sum b_{j}^{2}$

ta'I ta $=b \sum_{i v} t a_{i v}^{2}+\underset{i^{\prime} j v}{\Sigma} t a_{i^{\prime} j v}^{2}$

$b^{\prime} B^{\prime} t a=\sum_{v} b a_{j v} \sum_{i} t a_{i^{\prime} j v}$ 
$\mathrm{ba}^{\prime} \psi_{3}^{*} \mathrm{ba}=k \mathrm{ba}^{\prime} \mathrm{ba}=k \sum_{j v} b a_{j v}^{2}$

$a^{\prime} U a=b K a^{\prime} a=b K \sum_{V}^{\Sigma} a_{v}^{2}$

tendo-se assim, na forma matricial

$\theta^{\prime} X^{\prime}\left[\begin{array}{l:ll}x_{5} & x_{6}\end{array}\right] \quad L^{-1}\left(\begin{array}{c}X_{5}^{\prime} \\ \hdashline \frac{x_{6}^{\prime}}{6}\end{array}\right) X \theta=n m^{2}+\tau^{\prime} R \tau+2 b^{\prime} N^{\prime} \tau+b^{\prime} \psi_{1}^{*} b+$

$+a^{\prime} U a+t a^{\prime} L t a+2 b a^{\prime} B^{\prime} t a+b a^{\prime} \psi_{3}^{*} b a=$

$=n m^{2}+\tau^{\prime} R \tau+2 b^{\prime} N^{\prime} \tau+v k b^{\prime} b+b K a^{\prime} a+t a^{\prime} L t a+$

$$
+2 b^{\prime} B^{\prime} t a+k b a^{\prime} b a
$$

b) $E\left[\varepsilon^{\prime}\left[x_{5}: x_{6}\right] L^{-1}\left[\begin{array}{c}x_{5}^{\prime} \\ \hdashline x_{6}^{\prime}\end{array}\right]\right.$ E]

devido as estruturas das matrizes $\mathrm{x}_{5}$ e $\mathrm{x}_{6}$, e de forma análoga ao desenvolvimento de (466.3) e fazendo-se

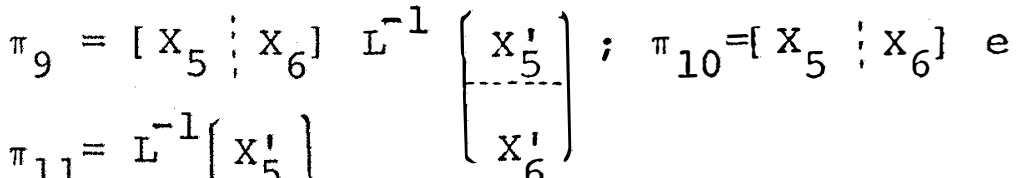

$$
\begin{aligned}
& \pi_{11}=L^{-1}\left(\begin{array}{c}
X_{5}^{\prime} \\
X_{6}^{2}
\end{array}\right)
\end{aligned}
$$

$\therefore \quad \operatorname{TR}\left(\pi_{9}\right)=\operatorname{TR}\left(\pi_{10} \pi_{1 I}\right)=\operatorname{TR}\left(\pi_{11} \pi_{10}\right)=\operatorname{TR}\left(L L^{-1}\right)=$

$$
=\operatorname{TR}\left(I_{[v(c+z)]}\right)=v^{\prime}(c+z)=\ell v
$$


$\log 0$

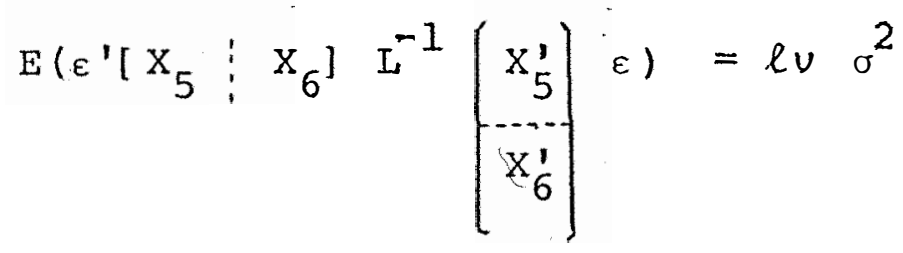

substituindo os valores $(467.4)$ e (467.5) em (467.2) tem-se:

$E\left(T A^{\prime} L^{-1} T A\right)=n m^{2}+\tau^{\prime} R \tau+2 b^{\prime} N^{\prime} \tau+v k b^{\prime} b+b K a^{\prime} a+$

$$
+t a^{\prime} L t a+2 b a^{\prime} B^{\prime} t a+k b a^{\prime} b a+\ell v \sigma^{2} \text { (467.6) }
$$

ii) $E\left(\frac{I}{C} B A^{\prime} B^{\prime} L^{-1} T A\right)=\frac{1}{c} E\left[Y^{\prime} X_{7} B^{\prime} L^{-1}\left[\begin{array}{c}X_{5}^{\prime} \\ \hdashline X_{6}^{\prime}\end{array}\right) Y\right]=$

$\left.=\frac{1}{c} E\left[\theta^{\prime} X^{\prime}+\varepsilon\right] X_{7} B^{\prime} L^{-1}\left[\begin{array}{c}X_{5}^{\prime} \\ x_{6}^{\prime}\end{array}\right][X \theta+\varepsilon]\right]=$

$=\frac{1}{c} \theta^{\prime} X^{\prime} X_{7} B^{\prime} L^{-1}\left(\begin{array}{c}X_{5}^{\prime} \\ \hdashline X_{6}^{\prime}\end{array}\right) X \theta+\frac{1}{c} E\left[\varepsilon^{\prime} X_{7} B^{\prime} L^{-1}\left(\begin{array}{c}X_{5}^{\prime} \\ \hdashline X_{6}^{\prime}\end{array}\right) \varepsilon\right]$ 
desenvolvendo por partes tem-se

a) $\frac{1}{c} \theta^{\prime} X^{\prime} X_{7} B^{\prime} L^{-1}\left(\begin{array}{c}x_{5}^{\prime} \\ \hdashline x_{6}^{\prime}\end{array}\right) x \theta$

onde $X^{\prime} X_{7}=\left(\begin{array}{c}E \\ D \\ F \\ G \\ B \\ W\end{array}\right)$

e fazendo

$$
\Psi=B^{\prime} L^{-1}\left(\begin{array}{c}
x_{5}^{\prime} \\
X_{6}^{\prime}
\end{array}\right) \quad X=
$$

$=\left[\begin{array}{lllll}\left.B^{\prime} L^{-1} e^{\prime} \quad B^{\prime} L^{-1} S^{\prime} \quad B^{\prime} L^{-1} V^{\prime} \quad B^{\prime} L^{-1} H^{\prime} \quad B^{\prime} L^{-1} L \quad B^{\prime} L^{-1} B\right]\end{array}\right.$

$\operatorname{mas} B^{\prime} L^{-1} e^{\prime}=E^{\prime} \quad B^{\prime} L^{-1} S^{\prime}=D^{\prime} \quad B^{\prime} L^{-1} V^{\prime}=\psi$

$$
B^{\prime} L^{-1} H^{\prime}=G^{\prime} \quad B^{\prime} L^{-1} L=B^{\prime} \quad B^{\prime} L^{-1} B=\psi_{3}^{*}
$$

$\Psi=\left[\begin{array}{llllll}I^{\prime} & D^{\prime} & \psi_{2}^{*} & G^{\prime} & B^{\prime} & \psi_{3}^{*}\end{array}\right]$ 


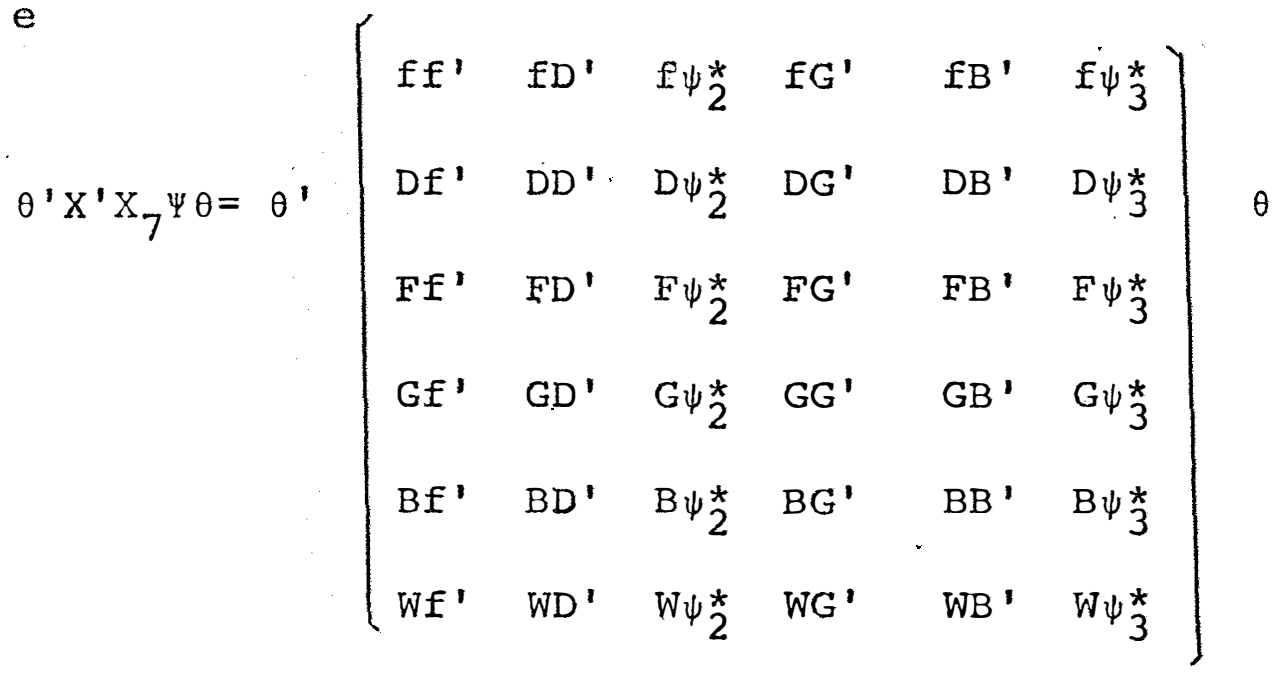

efetuando-se os produtos e de acordo com as restrições, têmse as seguintes matrizes nulas.

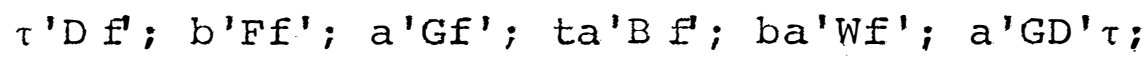

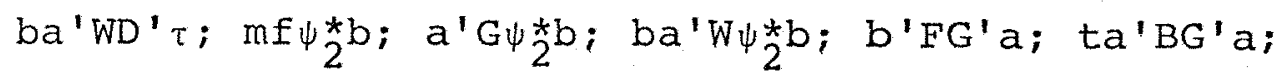
ba'WG'a; mfB'ta; $\operatorname{mf} \psi_{3}^{*} \mathrm{ba} ; \tau^{\prime} \mathrm{D} \psi{ }_{3}^{*} \mathrm{ba} ; \mathrm{b}^{\prime} \mathrm{F} \psi_{3}^{*} \mathrm{ba} ; \mathrm{a}^{\prime} \mathrm{G} \psi{ }_{3}^{*} \mathrm{ba}$;

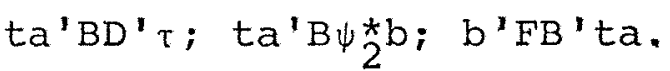
e as matrizes não nulas.

$m f f^{\prime} m=k n m^{2}$

$$
\begin{aligned}
& \tau^{\prime} \mathrm{DD}^{\prime} \tau=v\left[\sum_{i} t_{i} \sum_{i j} t_{i^{\prime} j}+\sum_{j}\left(\sum_{i^{\prime}} t_{i^{\prime} j}\right)^{2}\right] \\
& b^{\prime} F D^{\prime}=K b^{\prime} N^{\prime} \tau=v K \sum_{j} b_{j} \sum_{i} t_{i^{\prime} j}
\end{aligned}
$$


$\tau^{\prime} D \psi_{2}^{* b}=k b^{\prime} N^{\prime} \tau=v k \underset{f}{\sum} b_{j} \underset{i^{\prime}}{\Sigma} t_{i^{\prime} j}$

$b^{\prime} F \psi_{2}^{*} b=k K v b^{\prime} b=k K v \xi b_{j}^{2}$

$a^{\prime} G^{\prime} a=b K^{2} a^{\prime} a=b K^{2} \sum_{v} a_{v}^{2}$

$t a^{\prime} B^{\prime} t a=\sum_{j v}\left(\sum_{i} t a_{i v}+\sum_{i} t a_{i j^{\prime} v^{\prime}}\right)^{2}$

$b a^{\prime} W B^{\prime} t a=K b a^{\prime} B^{\prime} t a=K \sum_{j v} b a_{j v} \quad \sum_{i}^{\prime} t a_{i^{\prime} j v}$

$t a^{\prime} \psi_{3}^{* b a}=k b^{\prime} B^{\prime} t a=k \underset{j v}{\Sigma} b a_{j v} \sum_{i} t a_{i^{\prime} j v}$

$\mathrm{ba}^{\prime} \mathrm{W} \psi_{3}^{*} \mathrm{ba}=k K \mathrm{ba} \cdot \mathrm{ba}=k K \sum_{j v}^{\Sigma} b a_{j v}^{2}$

assim na forma matricial tem-se

$\frac{1}{c} \theta^{\prime} X^{\prime} X_{7} \psi \theta=\frac{1}{c}\left[m f f^{\prime} m+\tau^{\prime} D D^{\prime} \tau+b^{\prime} F D^{\prime} \tau+\tau^{\prime} D \psi \psi_{2}^{*} b+b^{\prime} F \psi_{2}^{*} b+\right.$

$$
\begin{aligned}
& \left.+a^{\prime} G G^{\prime} a+t a^{\prime} B B^{\prime} t a+b a^{\prime} W B^{\prime} t a+t a^{\prime} B \psi_{3}^{*} b a+b a^{\prime} W \psi_{3}^{\star} b a\right\}= \\
& =\frac{1}{c}\left\{K n m^{2}+\tau^{\prime} D D^{\prime} \tau+(K+k) b^{\prime} N^{\prime} \tau+k K v b^{\prime} b+b K^{2} a^{\prime} a+\right. \\
& \left.+t a^{\prime} B B^{\prime} t a+(K+k) b a^{\prime} B^{\prime} t a+k K b a{ }^{\prime} b a\right\}
\end{aligned}
$$

b) $\frac{1}{c} E\left[\varepsilon^{\prime} X_{7} B^{\prime} \bar{L}^{-1}\left(\begin{array}{c}X_{5}^{\prime} \\ \hdashline X_{6}^{\prime}\end{array}\right) \varepsilon\right)$ 
fazendo

$$
\pi_{12}=X_{7} B^{\prime} L^{-1}\left(\begin{array}{c}
X_{5}^{\prime} \\
\hdashline x_{6}^{\prime}
\end{array}\right) ; \pi_{13}=X_{7} B^{\prime} L^{-1} \text { e } \pi_{14}=\left(\begin{array}{c}
x_{5}^{\prime} \\
\hdashline x_{6}^{\prime}
\end{array}\right)
$$

$\operatorname{TR}\left(\pi_{12}\right)=\operatorname{TR}\left(\pi_{14} \pi_{13}\right)=\operatorname{TR}\left(\mathrm{BB}^{\prime} \mathrm{L}^{-1}\right)=\ell v$

$\therefore \quad \frac{1}{c} E\left(\varepsilon^{\prime} \pi_{12} \varepsilon\right)=\frac{l}{c} \operatorname{TR}\left(\pi_{12}\right) \sigma^{2}=\frac{l v}{c} \sigma^{2}$

substituindo $(467.8)$ e $(467.9)$ em $(467.7)$, tem-se

$\therefore \frac{1}{c} E\left(B A^{\prime} B^{\prime} L^{-1} T A\right)=\frac{1}{c}\left\{K \mathrm{~nm}^{2}+\tau{ }^{\prime} D D^{\prime} \tau+(K+k) b^{\prime} N^{\prime} \tau+k K v^{\prime} b^{\prime} b+\right.$ $\left.+b K^{2} a^{\prime} a+t a^{\prime} B B^{\prime} t a+(K+k) b a^{\prime} B^{\prime} t a+k K b a^{\prime} b a\right\}+\frac{\ell v}{c} \sigma^{2}$ $\ldots(467,10)$

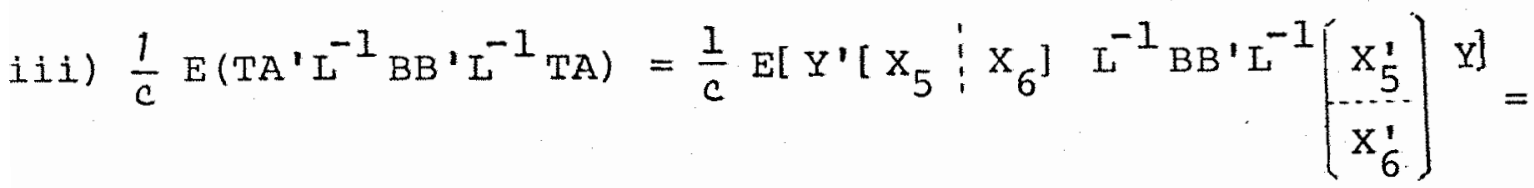
$=\frac{1}{c} E\left[\left(\theta^{\prime} X^{\prime}+\varepsilon^{\prime}\right)\left[X_{5}: X_{6}\right] L^{-1} B^{\prime} L^{-1}\left[\begin{array}{c}X_{5}^{\prime} \\ \hdashline X_{6}^{\prime}\end{array}\right](X \theta+\varepsilon)\right]$

desenvolvendo o produto e aplicando a esperança, têm-se

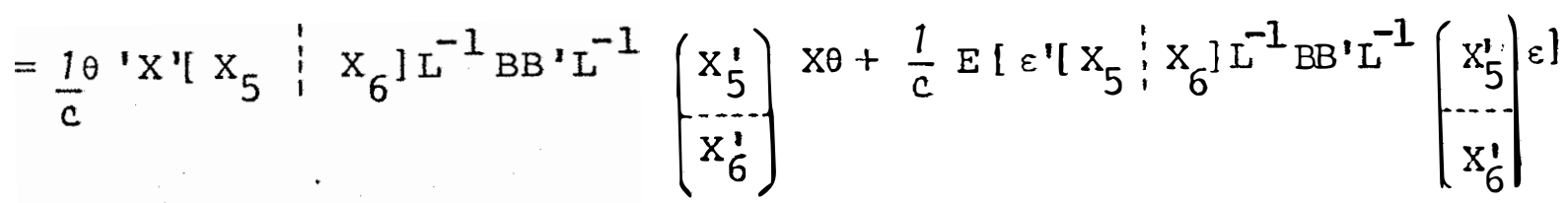


mas

$$
\begin{aligned}
& \Psi=B^{\prime} I^{-1}\left[\begin{array}{c}
X_{5}^{\prime} \\
\vdots X_{6}^{\prime}
\end{array}\right] x=\left[\begin{array}{llllll}
E^{\prime} & D^{\prime} \psi_{2}^{*} & G^{\prime} & B^{\prime} & \psi_{3}^{*}
\end{array}\right] \\
& =\frac{1}{c} \theta^{\prime} \Psi^{\prime} \Psi \theta+\frac{1}{c} E\left[\varepsilon^{\prime}\left[X_{5}: X_{6}\right] \quad L^{-1} B_{B}^{\prime} L^{-1}\left[\begin{array}{c}
X_{5}^{\prime} \\
\hdashline X_{6}^{\prime}
\end{array}\right] \varepsilon\right]
\end{aligned}
$$

resolvendo por partes, têm-se

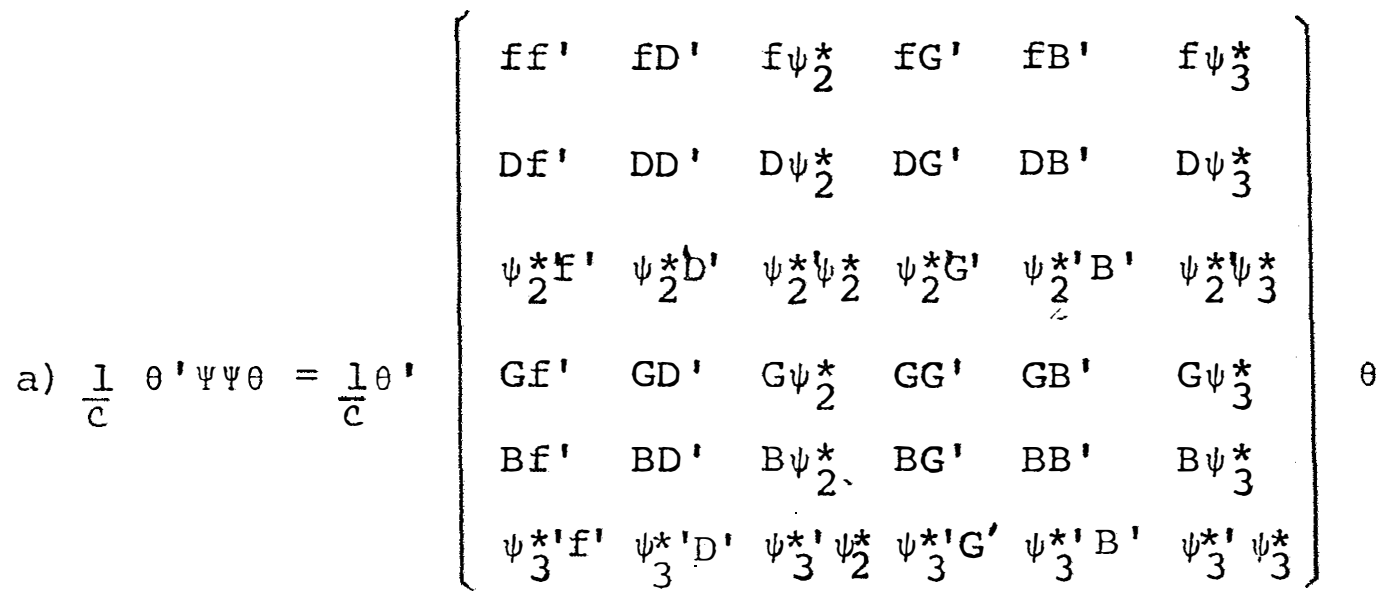

efetuando-se o produto e utilizando-se das restrições, têm-se as seguintes matrizes nulas.

$\tau^{\prime} D f^{\prime} m ; b^{\prime} \psi_{2}^{*} f^{\prime} m ; a^{\prime} f^{\prime} m ;$ ta'Bf'm; ba' $\psi_{3}^{*} f^{\prime} m ;$

$a^{\prime} G D^{\prime} \tau ; \operatorname{ta} B^{\prime} D^{\prime} \tau ; b a^{\prime} \psi_{3}^{*} D^{\prime} \tau ; \operatorname{ta} B \psi{ }_{2}^{*} b ; a^{\prime} G \psi{ }_{2}^{*} b ;$

ta'BG'a; ba' $\psi_{3}^{*} \mathrm{G}^{\prime} \mathrm{a} ; \mathrm{ba} \mathrm{a}^{\prime} \psi_{3}^{*} \psi_{2}^{*} \mathrm{~b}$. 
e as seguintes matrizes não nulas.

$m f f^{\prime} m=K n m^{2}$

$\left.\tau^{\prime} D^{\prime} \tau=v\left[\sum_{i} t_{i} \sum_{i^{\prime} j^{\prime}} t_{i^{\prime} j}+\sum_{j} \mid \sum_{i^{\prime}} t_{i^{\prime} j}\right)^{2}\right]$

$b^{\prime} \psi_{2}^{*} D^{\prime} \tau=k b^{\prime} N^{\prime} \tau=k v \sum_{J}^{\Sigma} b_{j} \sum_{i}^{i^{\prime}} t_{i} j$

$t a^{\prime} B B^{\prime} t a=\sum_{j v}\left(\sum_{i} t a_{i v}+\sum_{i} t a_{i} j v\right)^{2}$

$\left.b^{\prime} \psi_{2}^{* \prime} \psi_{2}^{*} \mathrm{~b}=v k^{2} \quad \mathrm{~b}^{\prime} \mathrm{b}=v k^{2} \sum_{j} b_{\}}\right\}$

$a^{\prime} G^{\prime} a=b K^{2} a^{\prime} a=b K^{2} \sum_{v} a_{v}^{2}$

$\mathrm{ba}^{\prime} \psi_{3}^{*} \mathrm{~B}^{\prime} \mathrm{ta}=k \mathrm{ba}^{\prime} \mathrm{B}^{\prime} t \mathrm{a}=k \sum_{j v} b a_{j v}{\stackrel{i}{i^{\prime}}} t a_{i^{\prime} j v}$

$\mathrm{ba}^{\prime} \psi_{3}^{* \prime} \psi_{3}^{*} \mathrm{ba}=k^{2} \mathrm{ba} \mathrm{a}^{\prime} \mathrm{ba}=k^{2} \underset{j v}{\Sigma} \quad b a_{j u}^{2}$

portanto na forma matricial tem-se

$\frac{1}{c} \theta^{\prime} \Psi^{\prime} \Psi \theta=\frac{1}{c}\left\{K n m^{2}+\tau^{\prime} D D^{\prime} \tau+2 k b^{\prime} N^{\prime} \tau+k^{2} u b^{\prime} b+b K^{2} a^{\prime} a+\right.$

$\left.+t a^{\prime} B^{\prime} t a+2 k b a^{\prime} B^{\prime} t a+k^{2} b a^{\prime} b a\right\}$

b) $\frac{1}{c} \quad E\left\{\varepsilon^{\prime}\left[X_{5}: X_{6}\right] \quad L^{-1} B^{\prime} L^{-1}\left[\begin{array}{c}X_{5}^{1} \\ \hdashline \\ X_{6}^{\prime}\end{array}\right] \varepsilon\right\}$ 
fazendo

$$
\pi_{15}=\left[\begin{array}{l:l}
X_{5} & X_{6}
\end{array}\right] \quad L^{-1} B^{\prime} L^{-1} \text { e } \pi_{16}=\left[\begin{array}{c}
X_{5}^{\prime} \\
\hdashline X_{6}^{\prime}
\end{array}\right]
$$

então

$\operatorname{TR}\left(\pi_{15} \cdot \pi_{16}\right)=\operatorname{TR}\left(\pi_{16} \cdot \pi_{15}\right)=\operatorname{TR}\left(\mathrm{BB}^{\prime} \mathrm{L}^{-1}\right)=\ell V$

$\therefore \frac{1}{c} \mathrm{E}\left\{\varepsilon^{\prime} \pi_{15} \cdot{ }_{16}{ }_{16}\right\}=\operatorname{TR}\left(\pi_{15} \cdot{ }_{16}\right) \sigma^{2}=\frac{\ell v}{c} \sigma^{2}$

substituindo $467.12 ; 467.13$ em 467.11 tem-se

$\frac{1}{\mathrm{c}} \mathrm{E}\left(\mathrm{TA} \mathrm{A}^{\prime} \mathrm{L}^{-1} \mathrm{BB}^{\prime} \mathrm{L}^{-1} \mathrm{TA}\right)=\frac{1}{\mathrm{c}^{2}} K \mathrm{n} \mathrm{m}^{2}+\tau^{\prime} \mathrm{DD}^{\prime} \tau+2 k \mathrm{~b}^{\prime} \mathrm{N}^{\prime} \tau+k^{2} v \mathrm{~b}^{\prime} \mathrm{b}+$

$$
\left.+b k^{2} a^{\prime} a+t a^{\prime} B^{\prime} t a+2 k b a^{\prime} B^{\prime} t a+k^{2} b a^{\prime} b a\right]+\frac{\ell v}{c} \sigma^{2}
$$

iv) $E($ SQtrat usual $)=E\left(\tau^{\prime} T\right)=E\left[T^{\prime} R^{-1} T-C o\right]$

$$
=E\left[T^{\prime} R^{-1} T\right]-E(C O)
$$

desenvolvendo por partes, tem-se 


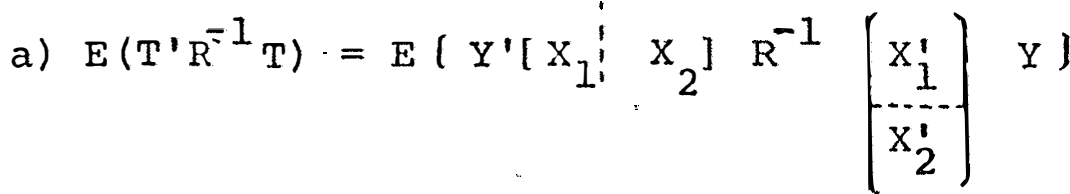

$$
\begin{aligned}
& =E\left[\left[\theta^{\prime} X^{\prime}+\varepsilon^{\prime}\right]\left[X_{1} \vdots X_{2}\right] R^{-1}\left[\begin{array}{c}
X_{1}^{\prime} \\
\hdashline X_{2}^{\prime}
\end{array}\right](X \theta+\varepsilon)\right]= \\
& =\theta^{\prime} x^{\prime}\left[\begin{array}{l:ll}
x_{1} & \left.x_{2}\right]
\end{array} R^{-1}\left[\begin{array}{c}
x_{1}^{\prime} \\
\hdashline x_{2}^{\prime}
\end{array}\right] \quad x \theta+\right.
\end{aligned}
$$

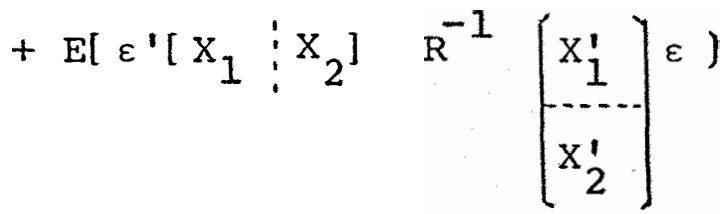

onde

a.1) $\theta^{\prime} \mathrm{x}^{\prime}\left[\begin{array}{l:lll}\mathrm{x}_{1} & \mathrm{x}_{2}\end{array}\right] \mathrm{R}^{-1}\left(\begin{array}{c}\mathrm{x}_{1}^{\prime} \\ \hdashline \mathrm{x}_{2}^{\prime}\end{array}\right) \mathrm{x} \theta=$

$=\theta^{\prime}\left(\begin{array}{cccccc}g R^{-1} g^{\prime} & g & g R^{-1} N & g R^{-1} P & g R^{-1} S & g R^{-1} D \\ g^{\prime} & R & N & P & S & D \\ N^{\prime} R^{-1} g^{\prime} & N^{\prime} & N^{\prime} R^{-1} N & N^{\prime} R^{-1} P & N^{\prime} R^{-1} S & N^{\prime} R^{-1} D \\ P^{\prime} R^{-1} g^{\prime} & P^{\prime} & P^{\prime} R^{-1} N & P^{\prime} R^{-1} P & P^{\prime} R^{-1} S & P^{\prime} R^{-1} D \\ S^{\prime} R^{-1} g^{\prime} & S^{\prime} & S^{\prime} R^{-1} N & S^{\prime} R^{-1} P & S^{\prime} R^{-1} S & S^{\prime} R^{-1} D \\ D^{\prime} R^{-1} g^{\prime} & D^{\prime} & D^{\prime} R^{-1} N & D^{\prime} R^{-1} P & D^{\prime} R^{-1} S & D^{\prime} R^{-1} D\end{array}\right) \theta$ 
efetuando-se os produtos e de acordo com as restrições impostas, têm-se as seguintes matrizes nulas.

$\tau^{\prime} g^{\prime} ; b^{\prime} N^{\prime} R^{-1} g^{\prime} ; a^{\prime} P^{\prime} ;$ ta's'; ba' $D^{\prime}$

e as matrizes não nulas.

$m g R^{-1} g^{\prime} m=n m^{2}$

$\tau^{\prime} R \tau=v\left[b \sum_{i} t_{i}^{2}+\sum_{i, j^{\prime}} t_{i}^{\prime} j^{\prime}\right]$

$b^{\prime} N^{\prime} \tau=\sum_{j} b_{j} \Sigma_{i^{\prime}} t_{i^{\prime} j}$

$b^{\prime} N^{\prime} R^{-1} N b=b^{\prime} \psi{ }_{1}^{*} b=v k b^{\prime} b=v k \sum_{j} b_{j}^{2}$

tem-se então

$\theta^{\prime} X^{\prime}\left[x_{1}: x_{2}\right] R^{-1}\left[\begin{array}{c}x_{1}^{\prime} \\ \hdashline x_{2}^{\prime}\end{array}\right] x \theta=n m^{2}+\tau \cdot R \tau+2 b^{\prime} N^{\prime} \tau+u k b^{\prime} b$

a.2) $E\left\{\varepsilon^{\prime}\left[\begin{array}{l:ll}X_{1} & X_{2}\end{array}\right] R^{-1}\left(\begin{array}{c}X_{1}^{\prime} \\ \hdashline X_{2}^{\prime}\end{array}\right) \varepsilon\right\}$

fazendo-se

$$
{ }_{17}=\left[x_{1}: x_{2}\right] ; \pi_{18}=R^{-1}\left(\begin{array}{c}
x_{1}^{\prime} \\
\hdashline x_{2}^{\prime}
\end{array}\right)
$$


então

$\operatorname{TR}\left(\pi_{17} \cdot \pi_{18}\right)=\operatorname{TR}\left(\pi_{18} \cdot \pi_{17}\right)=\operatorname{TR}\left(\mathrm{R}^{-1} \mathrm{R}\right)=\operatorname{TR}\left(\mathrm{I}_{(\ell)}\right)=\ell$

$\therefore \quad E\left(\varepsilon^{\prime} \pi 17 \cdot \pi 18^{\varepsilon}\right)=\operatorname{TR}(\pi 17 \cdot \pi 18) \quad[1+(v-1) \rho] \sigma^{2}$

$$
=\ell\left[1+|v-1| \rho \sigma^{2}\right.
$$

substituindo 467.16 e 467.17 em 467.15 , tem-se

$E\left(T^{\prime} R^{-1} T\right)=n m^{2}+\tau^{\prime} R \tau+2 b^{\prime} N^{\prime} \tau+v k b^{\prime} b+\ell[1+(v-1) \rho] \sigma^{2}$

e substituindo $46718 ; 4614$ em 46714 , tem-se

$E($ SQtrat $\underset{\text { usual }}{ })=\tau^{\prime} R \tau+2 b^{\prime} N^{\prime} \tau+v k b^{\prime} b+(l-1)[1+(v-1) \rho] \sigma^{2}$

retornando em 467.1 e substituindo os valores $467.6 ; 467.10$; 46714;467.19; 466.4; 461.4, tem-se na forma matricial:

$E($ SQtxa $)=t a^{\prime} L t a+b a^{\prime} B^{\prime} t a-b^{\prime} N^{\prime} \tau-v k b^{\prime} b+(l-1)(v-1)(1-\rho) \sigma^{2}$ $\ldots(467.20)$ 
our a inda

$E($ SQtxa $)=b \sum_{i v} t a_{i v}^{2}+\sum_{i^{\prime} j v} t a_{i^{\prime} j v}^{2}+\sum_{j v} b a_{j v} \sum_{i^{\prime}} t a_{i^{\prime} j v}-v \sum_{j} b_{j} \sum_{i^{\prime}} i^{\prime} j^{-}$

$$
-v k \quad \Sigma_{j} b_{j}^{2}+(\ell-1)(v-1)(1-p) \sigma^{2}
$$

$\therefore E(Q M t x a)=(1-\rho) \sigma^{2}+\frac{1}{(\ell-1 T T V-1 T}\left\{t a^{\prime} L t a+b a^{\prime} B^{\prime} t a-b^{\prime} N^{\prime} \tau-\right.$

$$
-v k b ' b \quad
$$

4.6.8. Interação bxa

De 452.16 temos

SQbxa $=\hat{b a} \hat{a}^{\prime} \mathrm{BA}=\frac{1}{\mathrm{c}} \mathrm{BA}^{\prime} \mathrm{BA}-\frac{1}{\mathrm{c}} \mathrm{TA}^{\prime} \mathrm{L}^{-1} \mathrm{BBA}-\mathrm{sQblocos}$

então

$E(S Q b x a)=\frac{I}{c} E\left(B A^{\prime} B A\right)-\frac{I}{c} E\left(T A^{\prime} L^{-1} B B A\right)-E(S Q b l o c o \stackrel{*}{S})(468,1)$

resolvendo por partes tem-se

i) $\frac{1}{c} E\left(B A^{\prime} B A\right)=\frac{1}{c} E\left(Y^{\prime} X_{7} X_{7}^{\prime} Y\right)=\frac{1}{c} E\left[\left(\theta^{\prime} X^{\prime}+\varepsilon^{\prime}\right) X_{7} X_{7}^{\prime}(X \theta+\varepsilon)\right]$

$$
=\frac{1}{c} \theta^{\prime} X^{\prime} X_{7} X_{7}^{\prime} X \theta+\frac{1}{c} E\left(\varepsilon^{\prime} X_{7} X_{7}^{\prime} \varepsilon\right)
$$


onde

a) $\frac{I}{c} \theta^{\prime} X^{\prime} X_{7} X_{7}^{\prime} X \theta=\frac{1}{c} \theta^{\prime}$

$\left[\begin{array}{cccccc}f f^{\prime} & f D^{\prime} & f F^{\prime} & f G^{\prime} & f B^{\prime} & f W^{\prime} \\ D f^{\prime} & D D^{\prime} & E F^{\prime} & D G^{\prime} & D B^{\prime} & D W^{\prime} \\ F f^{\prime} & F D^{\prime} & F F^{\prime} & F G^{\prime} & F B^{\prime} & F W^{\prime} \\ G f^{\prime} & G D^{\prime} & G F^{\prime} & G G^{\prime} & G B^{\prime} & G W^{\prime} \\ B f^{\prime} & B D^{\prime} & B F^{\prime} & B G^{\prime} & B B^{\prime} & B W^{\prime} \\ W f^{\prime} & W D^{\prime} & W F^{\prime} & W G^{\prime} & W B^{\prime} & W W^{\prime}\end{array}\right] \theta$

efetuando-se os produtos e de acordo com as restrições têm-se as seguintes matrizes nulas.

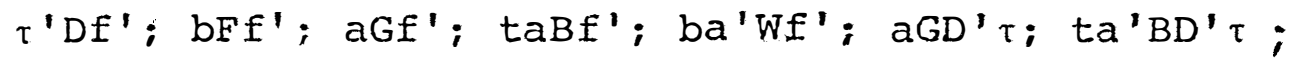

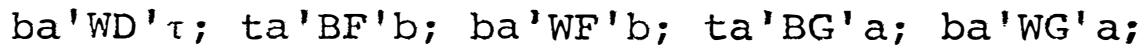

e as matrizes não nulas.

$m f^{\prime} m=K n m^{2}$

$\tau^{\prime} D^{\prime} \tau=v\left[\sum_{i} t_{i} \sum_{i j} t_{i} j^{\prime}+\sum_{j}\left(\sum_{i} t_{i^{\prime} j}\right)^{2}\right]$

$b^{\prime} F D^{\prime} \tau=K b^{\prime} N^{\prime} \tau=v K \sum_{j} b_{j} \sum_{i} t_{i^{\prime} j}$

$b^{\prime} F F^{\prime} b=u K^{2} b^{\prime} b=u K^{2} \sum_{J} b_{j}^{2}$

$a^{\prime} G G^{\prime} a=b K^{2} a^{\prime} a=b K^{2} \underset{v}{\mathbb{Z}} a_{v}^{2}$

$t a^{\prime} B^{\prime} t a=\sum_{j v}\left[\sum_{i} t a_{i v}+\sum_{i^{\prime}} t a_{i^{\prime} j u}\right]^{2}$ 
$b^{\prime} W^{\prime} t a=k b^{\prime} B^{\prime} t a=k \underset{J v}{\sum_{V}} b a_{j v} \sum_{i}, t a_{i} j v$

$\mathrm{ba}^{\prime} \mathrm{WW} \cdot \mathrm{ba}=k^{2} \mathrm{ba} \cdot \mathrm{ba}=k^{2} \sum_{j v} b a_{j v}^{2}$

tem-se assim

$\frac{1}{c} \theta^{\prime} X^{\prime} X X_{7}^{\prime} X \theta=\frac{1}{c}\left\{K n m^{2}+\tau{ }^{\prime} D D^{\prime} \tau+2 K b^{\prime} N^{\prime} \tau+v K^{2} b^{\prime} b+\right.$

$$
\left.+b K^{2} a^{\prime} a+t a B B^{\prime} t a+2 K b a^{\prime} B^{\prime} t a+K^{2} b a^{\prime} b a\right\} \text { (4683) }
$$

b) $\frac{1}{c} E\left(\varepsilon^{\prime} X_{7} X_{7}^{\prime} \varepsilon\right)=\frac{1}{c} \operatorname{TR}\left(X_{7} X_{7}^{\prime}\right) \sigma^{2}$

onde $\operatorname{TR}\left(X_{7} X_{7}^{\prime}\right)=b K v$

$\therefore \frac{l}{c} E\left(\varepsilon^{\prime} X_{7} X_{7}^{\prime} \varepsilon\right)=\frac{b K v}{c} \sigma^{2}(4684)$

substituindo 468.3 e 468.4 em 468.2 , tem-se

$\frac{1}{c} E\left(B A^{\prime} B A\right)=\frac{b K v}{c} \sigma^{2}+\frac{1}{c}\left\{K n m^{2}+\tau^{\prime} D D^{\prime} \tau+2 K b^{\prime} N^{\prime} \tau+v K^{2} b^{\prime} b+\right.$ $\left.+b K^{2} a^{\prime} a+t a ́ B B^{\prime} t a+2 K b a^{\prime} B^{\prime} t a+K^{2} b a^{\prime} b a\right\}$

ii) $\frac{1}{c} \mathrm{E}\left(\mathrm{TA}^{\prime} \mathrm{L}^{-1} \mathrm{BBA}\right)=\frac{1}{\mathrm{c}} \mathrm{E}\left(\mathrm{BA}^{\prime} \mathrm{B}^{\prime} \mathrm{L}^{-1} \mathrm{TA}\right)$

cujo valor è visto em 467.10. 
iii) $E(\text { Sóbloco } \stackrel{*}{S})^{\prime}=E\left(\hat{b}^{\prime} B\right)$

substituindo o valor de $\hat{b}^{\prime}=\left[B^{\prime} A^{-1}-\hat{\tau}^{\prime} N A^{-1}-\hat{m} c A^{-1}\right]$

tem-se

$$
\begin{gathered}
\hat{b} \cdot B=B^{\prime} A^{-1} B-\hat{\tau}^{\prime} N A^{-1} B-\hat{m}_{C A}^{-1} B \\
\text { mas } \hat{m} C A^{-1} B=C o ; \\
B^{\prime} A^{-1} B-C O=\text { SQblocos; } \\
N A^{-1} B=T-Q \\
\therefore \hat{\tau}^{\prime} N A^{-1} B=\hat{\tau}^{\prime} T-\hat{\tau}^{\prime} Q \\
\hat{\tau}^{\prime} T=\text { sQtrat usual } \\
\hat{\tau}^{\prime} Q=\text { sQtrat }{ }_{\text {ajustado }}
\end{gathered}
$$

entล̃o

$E($ SQblocós $)=E[$ SQblocos $]-E\left[\right.$ sQtrat $\left._{\text {usual }}\right]+E\left[\right.$ sQtrat $\left._{\text {ajust }}\right]$

substituindo os valores $463.4 ; 467.19 ; 462.6$, tem-se

$E\left(S Q b l o c{ }^{*}\right)=c u b^{\prime} b+(b-1)[1+(v-1) \rho] \sigma^{2}$ 
substituindo os valores $468.5 ; 467.10 ; 468.6$ em 468.1 têm-se na forma matricial.

$E(S Q b \times a)=\left[\frac{b K v}{c}-\frac{\ell v}{c}-(b-1)\right] \sigma^{2}-(b-1)(v-1) p \sigma^{2}+b^{\prime} N^{\prime} \tau+$ $+v k b^{\prime} b+b a^{\prime} B^{\prime} t a+k b a^{\prime} b a$

mas

$\left[\frac{b K v}{c}-\frac{\ell v}{c}-(b-1)\right]=\left[\frac{v}{c}(b K-\ell)-(b-1)\right]=(v-1)(b-1)$

observe que

$b k-l=b(c+k)-l=b c+b k-l=b c+z-l=(b-1) c$.

assim

$E(S Q b \times a)=(v i-1)(b-1)(1-\rho) \sigma^{2}+b^{\prime} N^{\prime} \tau+u k b^{\prime} b+b a^{\prime} B^{\prime} t a+$

$$
+K b a^{\prime} b a
$$

ou ainda

$$
\begin{aligned}
E(S Q b x a)= & (v-1)(b-1)(1-\rho) \sigma^{2}+v \sum_{j} b \sum_{i^{\prime}} t_{i^{\prime} j^{\prime}}+v k \sum_{j} b_{j}^{2}+ \\
& +k \sum_{j v} b a_{j v}^{2}+\sum_{j v} b a_{j v} \sum_{i^{\prime}}^{\Sigma} t a_{i^{\prime} j v} \quad(468,8)
\end{aligned}
$$


.85 .

e

$E(Q M b x a)=(1-\rho) \sigma^{2}+\frac{1}{|v-1| \mid b-1 T}\left\{b^{\prime} N^{\prime} \tau+v k b^{\prime} b+b a^{\prime} B^{\prime} t a+K b a^{\prime} b a\right\}$

4.6.9. Total

sQTotal $=Y^{\prime} Y-$ Co

$(469.1)$

$E(S Q T o t a I)=E\left(Y^{\prime} Y\right)-E(C O)$

$(469.2)$

resolvendo por partes tem-se

i) $E\left(Y^{\prime} Y\right)=E\left(Y^{\prime} I Y\right)=E\left\{\left[\theta^{\prime} X^{\prime}+\varepsilon^{\prime}\right] I[X \theta+\varepsilon]\right\}$

$$
=\theta^{\prime} X^{\prime} I X \theta+E\left\{\varepsilon^{\prime} I \quad \varepsilon\right\} \quad \text { (469.3) }
$$

onde

a) $\theta^{\prime} X^{\prime} I X \theta=\theta^{\prime}\left(\begin{array}{llllll}n & g & c & d & e & f \\ g^{\prime} & R & N & P & S & D \\ C^{\prime} & N^{\prime} & A & K & V & F \\ d^{\prime} & P^{\prime} & K^{\prime} & U & H & G \\ e^{\prime} & S^{\prime} & V^{\prime} & H^{\prime} & \text { L } & B \\ f^{\prime} & D^{\prime} & F^{\prime} & G^{\prime} & B^{\prime} & W\end{array}\right] \theta$ 
efetuando-se os produtos e de acordo com as restrições têm-se:

$\theta^{\prime} X^{\prime}$ I $X \theta=n m^{2}+\tau^{\prime} R \tau+2 b^{\prime} N^{\prime} \tau+b^{\prime} A b+a^{\prime} U a+$

$$
+t a^{\prime} L t a+2 t a{ }^{\prime} B b a+b a^{\prime} W b a
$$

b) $E\left(\varepsilon^{\prime} I \varepsilon\right)=\operatorname{TR}(I(b K v)) \sigma^{2}=b K V \quad \sigma^{2}$ (469.5)

substituindo $469.4 ; 469.5$ e 461.4 em 469.2

$E($ SQTotal $)=[(6 K v-1)-(v-1) \rho] \sigma^{2}+\tau^{\prime} R \tau+2 b^{\prime} N^{\prime} \tau+b^{\prime} A b+$

$$
+a^{\prime} U a+\text { ta'Lta+ ba'Wba+2ta'Bba }
$$

ou ainda

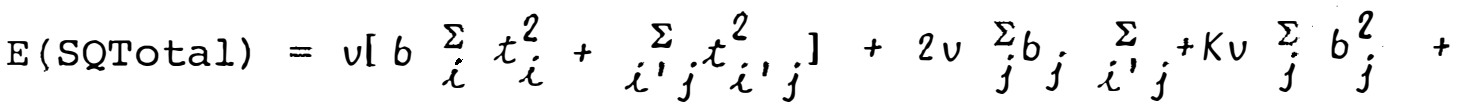

$$
\begin{aligned}
& +b k \sum_{v} a_{v}^{2}+b \sum_{i v} t a_{i v}^{2}+i_{j v} t a_{i}^{2}, \sum_{j v}+k \sum_{j v}^{\Sigma} b a_{j v}^{2}+ \\
& +2 \sum_{j v} b a_{j v} \sum_{i} t a_{i j} j v+[b K v-(1+(v-1) \rho)] \sigma^{2}
\end{aligned}
$$

$$
\text { 4.6.10. Resíduo (b) }
$$

$E($ SQRes $(b))=E$ SQTotal - SQParcelas - SQParâmetros (b) 
substituindo $469.6 ; 464.4 ; 466.4 ; 467.21 ; 468.7$ tem-se

$E(\operatorname{SQRes}(b))=(v-1)[b(k-1)-(\ell-1)](1-\rho) \sigma^{2}$

$(4610.1)$

$\therefore E(Q M R e s(b))=(1-\rho) \sigma^{2}$ 
.88,

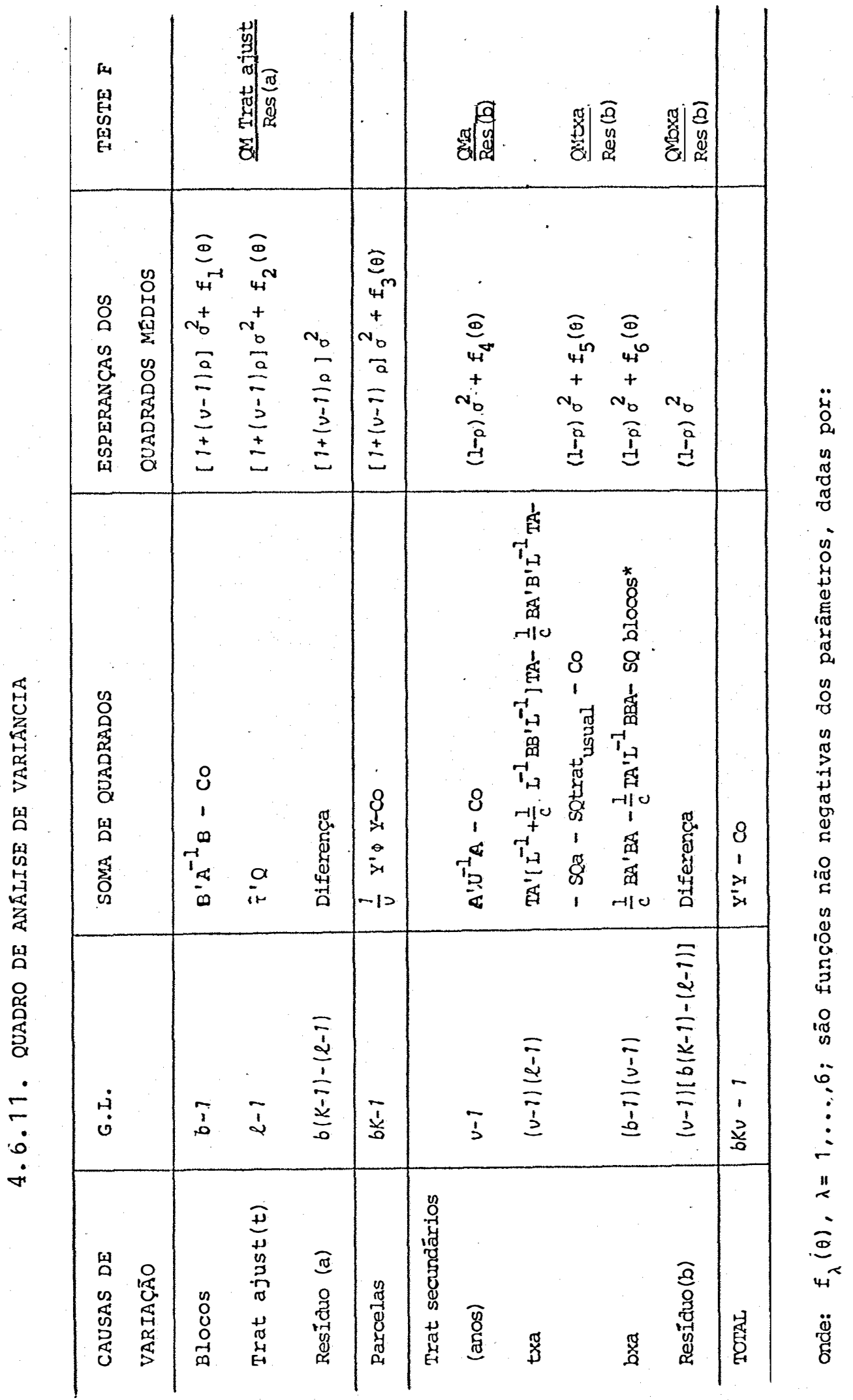




$$
\begin{aligned}
& f_{1}(\theta)=\left\{\tau^{\prime} R \tau-\tau^{\prime} Q+2 b^{\prime} N^{\prime} \tau+b^{\prime} A b\right\} /(b-1) \\
& f_{2}(\theta)=\left\{\tau^{\prime} Q\right\} /(\ell-1) \\
& f_{3}(\theta)=\left\{\tau^{\prime} R \tau+2 b^{\prime} N^{\prime} \tau+b^{\prime} A b\right\} /(b K-1) \\
& f_{4}(\theta)=\left\{a^{\prime} U a\right\} /(v-1) \\
& f_{5}(\theta)=\left\{t a a^{\prime} L t a+b a^{\prime} B^{\prime} t a-b^{\prime} N^{\prime} \tau-v k b b^{\prime} b\right\} /(l-1)(v-1) \\
& f_{6}(\theta)=\left\{K b a a^{\prime} b a+b a^{\prime} B^{\prime} t a+b^{\prime} N^{\prime} \tau+v k b b^{\prime} b\right\} /(v-1)(b-1)
\end{aligned}
$$

4.7. Dispersão para Tratamentos

\subsubsection{Tratamentos Principais}

Para a determinação da matriz de dispersão para tratamentos principais, considerou-se como de modo usual

$D(\hat{\tau})=E\left[[\hat{\tau}-E(\hat{\tau})][\hat{\tau}-E(\hat{\tau})]^{\prime}\right]$

onde por $44.11 ; \hat{\tau}=M^{-1} Q$ e por $44.6 ; Q=T-N A^{-1} B$

$$
\therefore \hat{\tau}=M^{-1} T_{-M}^{-1} N^{-1} B
$$

$$
\operatorname{mas} T=\left(\begin{array}{c}
x_{1}^{\prime} \\
x_{2}^{\prime}
\end{array}\right) \quad Y=\left[\begin{array}{c}
x_{1}^{\prime} \\
\hdashline x_{2}^{\prime}
\end{array}\right]
$$

$(X \theta+\varepsilon)$ 


$$
\begin{aligned}
& B=X_{3}^{\prime} Y=X_{3}^{\prime}(X \theta+\varepsilon) \\
& \therefore \hat{\tau}=M^{-1}\left[\begin{array}{l}
X_{1}^{\prime} \\
\hdashline \\
X_{2}^{\prime}
\end{array}\right](X \theta+\varepsilon)-M^{-1} N A^{-1} X_{3}^{\prime}(X \theta+\varepsilon) \\
& \hat{\tau}=M^{-1}\left[\begin{array}{l}
X_{1}^{\prime} \\
X_{2}^{\prime}
\end{array}\right] \quad X \theta-M^{-1} N A^{-1} X_{3}^{\prime} X_{\theta}+M^{-1}\left[\begin{array}{l}
X_{1}^{\prime} \\
\hdashline \\
X_{2}^{\prime}
\end{array}\right] \varepsilon-M^{-1} N A^{-1} X_{3}^{\prime} \varepsilon
\end{aligned}
$$

e desse modo

$$
E(\hat{\tau})=M^{-1}\left(\begin{array}{l}
x_{1}^{\prime} \\
x_{2}^{\prime}
\end{array}\right) x \theta-M^{-1} N^{-1} X_{3}^{\prime} X \theta
$$

assim

$$
\hat{\tau}-E(\hat{\tau})=M^{-1}\left(\left[\begin{array}{c}
X_{1}^{\prime} \\
\hdashline X_{2}^{\prime}
\end{array}\right)-N A^{-1} X_{3}^{\prime}\right) \varepsilon=M^{-1} \Omega \varepsilon
$$

fazendo

$\Omega=\left(\begin{array}{c}x_{1}^{\prime} \\ x_{2}^{\prime}\end{array}\right)-N A^{-1} x_{3}^{\prime}$

$\therefore D(\hat{\tau})=E\left[M^{-1} \Omega \varepsilon \varepsilon^{\prime} \Omega^{\prime} M^{-1}\right]$ 
Determinando-se a esperança temos.

$\boldsymbol{C}(\hat{\tau})=[I+(u-1) \rho] \sigma^{2} \boldsymbol{M}^{-1} \Omega \Omega M^{-1} \boldsymbol{M}^{\prime}$

efetuando-se o produto $\Omega \Omega^{\prime}$ e lembrandorse

$$
\begin{aligned}
& \left(\begin{array}{c}
\mathrm{x}_{1}^{\prime} \\
\hdashline \mathrm{x}_{2}^{\prime}
\end{array}\right)\left[\mathrm{x}_{1}: \mathrm{x}_{2}\right]=\mathrm{R} \\
& \left(\begin{array}{c}
\mathrm{x}_{1}^{\prime} \\
\hdashline \mathrm{x}_{2}^{\prime}
\end{array}\right) \mathrm{x}_{3}=\mathrm{N} \\
& \mathrm{x}_{3}^{\prime} \mathrm{x}_{3}=\mathrm{A}
\end{aligned}
$$

temos

$$
\begin{aligned}
& \Omega \Omega^{\prime}=R-N A^{-1} N^{\prime}-N A^{-1} N^{\prime}+N A^{-1} A A^{-1} N^{\prime} \\
& \Omega \Omega^{\prime}=R-N A^{-1} N^{\prime}=C .
\end{aligned}
$$

Desse modo $(471.4)$

$$
\sigma(\hat{\tau})=[1+(v-1) \rho] \sigma^{2} M^{-1} C M^{-1}
$$


, 92.

once

$$
M^{-1} \mathbf{C} \mathbf{M}^{-1}=\frac{1}{k}\left(\begin{array}{ccccc}
D_{1}^{*} & D_{2}^{*} & D_{2}^{*} & \ldots & D_{2}^{*} \\
D_{2}^{* 1} & D_{3}^{*} & D_{4}^{*} & \ldots & D_{4}^{*} \\
D_{2}^{* \prime} & D_{4}^{* \prime} & D_{3}^{*} & \ldots & D_{4}^{*} \\
\ldots & \ldots & \ldots & \ldots & \ldots \\
D_{2}^{* \prime} & D_{4}^{* 1} & D_{4}^{* \prime} & \ldots & D_{3}^{*}
\end{array}\right)
$$

sendo

$$
\begin{aligned}
& D_{1}^{*}=a_{s t}=\left\{\begin{array}{l}
\frac{k-1}{b v} \text { para } s=t \\
-\frac{1}{b v} \text { para } s \neq t
\end{array}\right. \\
& D_{2}^{*}=-\frac{1}{b v} E_{(c \times k)} \\
& D_{3}^{*}=a_{s t}=\left\{\begin{array}{l}
\frac{k}{v}+\frac{k}{c v}-\frac{k}{b c v}-\frac{1}{b v} \text { para sara s }=t \\
\frac{k}{c v}-\frac{1}{b v}-\frac{k}{b c v}
\end{array}\right. \\
& D_{4}^{*}=-\frac{k+c}{b c v} \mathbf{E}_{(k \times k)} \text { para }
\end{aligned}
$$


Assim, na diferença entre duas médias de efeitos es timados de tratamentos principais, seräoconsideradosos seguin tes casos:

a) Entre dois tratamentos comuns:

$$
\begin{aligned}
v\left(\hat{t}_{i}-\hat{t}_{i}\right) & =v\left(\hat{t}_{i}\right)+v\left(\hat{t}_{i}\right)-2 \operatorname{cov}\left(\hat{t}_{i} ; \hat{t}_{i}\right)= \\
& =\frac{1}{k}\left[\frac{k-1}{b v}+\frac{k-1}{b v}-2\left(-\frac{1}{b v}\right)\right][1+(v-1) \rho] \sigma^{2}= \\
& =\frac{2}{b v}[1+(v-1) 0] \sigma^{2}
\end{aligned}
$$

assim

$$
\hat{v}\left(\hat{t}_{i}-\hat{t}_{i}\right)=\frac{2}{b v} \quad \operatorname{QMRes}(a)
$$

b) Entre um tratamento comum e um regular:

$$
\begin{aligned}
v\left(\hat{t}_{i}-\hat{t}_{i^{\prime} j}\right)= & v\left(\hat{t}_{i}\right)+v\left(\hat{t}_{i^{\prime} j}\right)-2 \operatorname{cov}\left(\hat{t}_{i}, \hat{t}_{i^{\prime} j}\right) \\
= & \frac{1}{K}\left[\left(\frac{K-1}{b v}\right)+\left(\frac{K}{v}+\frac{K}{c v}-\frac{1}{b v}-\frac{K}{b c v}\right)-2\left(\frac{-1}{b v}\right)\right] \cdot \\
& .[1+(v-1) \rho] \sigma^{2} \\
= & \frac{1}{v}\left(1+\frac{1}{b}+\frac{1}{c}-\frac{1}{b c}\right)[1+(v-1) \stackrel{\rho}{b}] \sigma^{2} \\
\therefore \nabla\left(z_{i}-z_{i^{\prime} j}\right)= & \frac{1}{v}\left[1+\frac{1}{b}+\frac{1}{c}-\frac{1}{b c}\right] \operatorname{QMRes}(a)
\end{aligned}
$$


.94 .

c) Entrè dois tratamentos regulares dentro do mesmo bloco.

$$
\begin{aligned}
& v\left(\hat{t}_{i^{\prime} j}-\hat{t}_{i^{\prime \prime} j^{\prime}}\right)=v\left(\hat{t}_{i^{\prime} j}\right)+v\left(\hat{t}_{i^{\prime} \prime j}\right)-2 \operatorname{cov}\left(\hat{t}_{i^{\prime} j} ; \hat{t}_{i^{\prime} \prime j^{\prime}}\right) \\
&=\frac{2}{k}\left\{\left[\frac{k}{v}+\frac{k}{c v}-\frac{1}{b v}-\frac{k}{b c v}\right]-\left[\frac{k}{c v}-\frac{k}{b c v}-\frac{1}{b v} 1\right\} .\right. \\
& {[1+(v-1) \rho] \sigma^{2}=} \\
&=\frac{2}{v}[1+(v-1) \rho] \sigma^{2} \\
& \therefore \hat{v}\left(\hat{t}_{i^{\prime} j}-\hat{t}_{i^{\prime} \prime j}\right)=\frac{2}{v} \operatorname{QMRes}(a)
\end{aligned}
$$

d) Entre dois tratamentos regulares em blocos diferentes:

$$
\begin{aligned}
& v\left(\hat{t}_{i^{\prime} j}-\hat{t}_{i^{\prime \prime}} j^{\prime}\right)=v\left(\hat{t}_{i^{\prime} j}\right)+v\left(\hat{t}_{i^{\prime}, j}\right)-2 \operatorname{COV}\left(t_{i^{\prime} j} ; t_{i^{\prime} j^{\prime}}\right) \\
& =\frac{1}{K}\left\{2\left[\frac{K}{v}+\frac{K}{c v}-\frac{1}{b v}-\frac{K}{b c v}\right]-2\left[-\frac{K+c}{b c v}\right]\right\} . \\
& =[1+|v-1| \rho] \sigma^{2} \\
& =\frac{2}{v}\left[1+\frac{1}{c}[1+(v-1) \rho] \sigma^{2}\right. \\
& \therefore \nabla\left(z_{i j^{\prime}} z_{\left.i^{\prime \prime} j^{\prime}\right)}=\frac{2}{v}\left(1+\frac{1}{c}\right) \operatorname{QMRes}(a)\right.
\end{aligned}
$$


e) Variância entre constraste de médias de efeitos estimados para tratamentos principais.

Seja $\circ$ contraste $\hat{\mathbf{Y}}=\mathbf{P}^{\prime} \hat{t}$, onde $\mathbf{P}^{\prime} \bar{e}$ a matriz de coeficientes do contraste, de dim $1 \times l$, assim como de forma usual, tem-se:

$$
\mathrm{V}(\hat{\mathbf{X}})=\mathbb{P}^{\prime} \mathbf{M}^{-1} \mathbf{P}\left[1+(v-1)_{\rho}\right] \sigma^{2}
$$

\subsubsection{Tratamentos Secundários}

Considerando como de forma usual, tem-se

$$
O(\hat{a})=E\left\{[\hat{a}-E(\hat{a})][\hat{a}-E(\hat{a})]^{\prime}\right\}
$$

onde, por 44.1.6, tem-se

$$
\hat{a}=U^{-1}\left[\mathbb{A}-d^{\prime} \hat{m}\right]=\left[U^{-1} \mathbb{A}-\hat{m} E_{(v \times])}\right]
$$

mas $A=X_{4}^{\prime} Y=X_{4}^{\prime}(X \theta+\varepsilon)$, então

$$
\begin{aligned}
& \hat{a}=U^{-1} X_{4}^{\prime} X \theta+U^{-1} X_{4}^{\prime} \varepsilon-\hat{m} E_{(v \times 1)} \\
& E(\hat{a})=U^{-1} X_{4}^{\prime} X \theta-\hat{m} E_{(v \times 1)} \\
& \therefore \hat{a}-E(\hat{a})=U^{-1} X_{4}^{\prime} \varepsilon
\end{aligned}
$$


assim para $(472.1)$, tem-se

$$
\begin{aligned}
\mathcal{O ( \hat { a } )} & =E\left\{U^{-1} X_{4}^{\prime} \varepsilon \varepsilon^{\prime} X_{4} U^{-1}\right\} \\
& =\left(\frac{1}{b K}\right)^{2} X_{4}^{\prime} E\left(\varepsilon \varepsilon^{\prime}\right) X_{4}
\end{aligned}
$$

onde

$X_{4}^{\prime} E\left(\varepsilon \varepsilon^{\prime}\right) X_{4}=b K\left[\begin{array}{cccc}\sigma^{2} & \rho \sigma^{2} & \ldots & \sigma^{2} \\ \rho \sigma^{2} & \sigma^{2} & \ldots & \sigma^{2} \\ \ldots & \ldots & \ldots & \ldots \\ \rho \sigma^{2} & \rho \sigma^{2} & & \sigma^{2}\end{array}\right]_{(v x v)}=b K W^{*}$

$\hat{c}(\hat{a})=\frac{1}{b K} W^{*}$

Assim para à variância da diferença entre duas mẹ dias de efeitos estimados de tratamentos secundários, tem-se $V\left(\hat{a}_{v}-\hat{a}_{v^{\prime}}\right)=\frac{2}{b K}(I-p) \sigma^{2}$

$\therefore \hat{v}\left(\hat{a}_{v}-\hat{a}_{v^{\prime}}\right)=\frac{2}{b K} \quad \operatorname{QMRes}(b)$ 
4.7.3. Dispersão para a Interação txa

Como a dispersão de txa, não tem uso prático pelo modelo adotado, pois o que interessa são comparações de médias de tratamentos dentro de anos e médias de anos dentro de tratamentos, o que necessitaria de uma reestruturação do modelo, deixamos de fazer esse desenvolvimento sugerindo esse tema para trabalhos futuros.

4.8. Ajuste de Médias

a) o ajuste para as médias de tratamentos comuns, não se faz necessário, por estarem repetidos em todos os blocos.

b) O ajuste para as médias de tratamentos regulares, é dado por:

$$
\hat{m}_{i^{\prime} j}=\hat{t}_{i j}+\hat{m}=\frac{T_{i} j}{v}-\frac{1}{c v}\left(\sum_{l}^{*} T_{i} \mid / j+\frac{1}{b c v} \sum_{l} T_{i}\right. \text { (4821) }
$$


c) O ajuste para as médias dos tratamentos secundārios, tambēm não se faz necessārio.

4.9. Comparações Múltip̣las

Pode-se usar quaisquer dos testes para comparações múltiplas. Neste trabalho optou-se pelo teste TUKEY, citado em PIMENTEL GOMES(1976).

$$
\text { D.M.S }=q \sqrt{\frac{1}{2} \hat{V}(\hat{\mathbf{Y}})}
$$

onde

D.M.S = é a diferença mînima significativa.

$q=e ́$ o valor da amplitude total estudentizada $\hat{\mathrm{V}}(\hat{\mathbf{Y}})=\overline{\mathrm{e}}$ a variância estimada do controle estimado $\hat{\mathbf{Y}}$, entre os efeitos médios.

Têm-se então as seguintes D.M.S. 


\subsubsection{Para os Tratamentos Principais}

a) Se $\hat{\mathbf{Y}}$, è o contraste entre dois tratamentos comuns, istoé,

$\hat{\mathbf{Y}}_{1}=\hat{m}_{t}-\hat{m}_{t_{i}}$

D.M.S ${ }_{1}=q \cdot \sqrt{\frac{\operatorname{QMRes}(a)}{b v}} ; q(\alpha, l, g \operatorname{graus} \operatorname{Res}(a))$

b) Se $\hat{\mathbf{Y}}$, ê o contraste entre um tratamento comum e um regular, isto é,

$\hat{\mathbf{x}}_{2}=\hat{\mathrm{m}}_{t}-\hat{\mathrm{m}}_{t_{i^{\prime} j}}$

D.M.S $2=q \cdot \sqrt{\frac{1}{2}\left(\frac{1}{v}+\frac{1}{b v}+\frac{1}{c v}-\frac{1}{b c v}\right) \operatorname{QMRes}(a)}$;

$q(\alpha, l, g r a u s R e s(a))$.

c) Se $\hat{Y}$, é o contraste entre dois tratamentos regulares dentro do mesmo bloco;

$$
\begin{aligned}
& \hat{\mathbf{x}}_{3}=\hat{\mathrm{m}}_{t_{i^{\prime} j}}-\hat{\mathrm{m}}_{t_{i^{\prime} \prime j}} \\
& \text { D.M.s. } \\
& =q \sqrt{\frac{\operatorname{lMRes}(a)}{v}} ; q(\alpha, l, \operatorname{graus} \operatorname{Res}(a)) ;
\end{aligned}
$$


d) Se $\hat{Y}$, ê o contraste entre dois tratamentos regulares em blocos diferentes;

$$
\hat{\mathbf{x}}_{4}=\hat{m}_{t_{i^{\prime} j}}-\hat{m}_{t}{ }_{i^{\prime} j^{\prime}}
$$

D.M.S $4=q \cdot \sqrt{\left(\frac{l}{v}+\frac{1}{c v}\right) \operatorname{QMRes}(a)} ; q(\alpha, l, g r a u s \operatorname{Res}(a))(4914)$

\subsubsection{Para os Tratamentos Secundārios}

Se $\hat{\mathbf{y}}, \mathbf{e}$ o contraste entre dois tratamentos secundā rios, (anos);

$$
\begin{aligned}
\hat{\mathbf{y}}_{5} & =\hat{\mathrm{m}}_{a_{i}}-\hat{\mathrm{m}}_{a_{v^{\prime}},} \\
\text { D.M. } & =q \cdot \sqrt{\frac{\text { QMRes }(b)}{b K}} ; q(\alpha, v, \text { graus Res }(b))(492.1)
\end{aligned}
$$


4.10. Testes sobre a Estrutura da Matriz de Covariâncias

Alēm das tradicionais hipóteses de normalidade, in dependência, aditividade, etc., em experimentos ...envolvendo parcelas subdivididas no tempo, faz-se tambēm necessário os testes de homogeneidade e de uniformidade das matrizes de covariância entre tratamentos secundārios dentro de cada tratamento principal, pois não é raro encontrar correlações entre a produção de vários anos sucessivos.

Quando não se verificam as hipóteses de homogeneidade e de uniformidade das matrizes de covariâncias, IEMMA (1981), citando GILL(1978) e LEAL(1979), recomenda como alternativa ao modelo univariado, o uso da análise de perfil abordada por LEAL(1979).

4.10.1. Teste de Homogeneidade para as Matrizes de Covariâncias

Adotaremos o critério de BOX(1950) abordado por DINIZ(1980), descrito por DANFORD et alii,(1960), dentre outros.

$$
\begin{aligned}
\text { Sendo но } & =\$_{1}=\$_{2}=\ldots=\$_{C}=\$ \text { e } \\
\text { Ha } & =\$_{i} \neq \$_{i^{\prime}} \text { para algum } i \neq i^{\prime}
\end{aligned}
$$


- Calcula-se inicialmente $\mathbf{G}_{i}=($ gss', c) que è a estima tiva não tendenciosa de $\ddagger_{i}, i \neq 1 \ldots . . c$ (matriz de covariância entre tratamentos secundārios, para o i-ésimo tratamento principal).

$$
\begin{aligned}
\mathbf{G}_{i}=g\left(s s^{\prime} c\right) & =(b-1)^{-1} \sum_{j}\left(y_{i j v}-y_{i=v^{-}} y_{. j u}+y_{. v}\right) x \\
& x\left(y_{i j v^{\prime}}-y_{i \cdot v^{\prime}}-y_{. j v^{\prime}}+y_{. . v^{\prime}}\right)
\end{aligned}
$$

onde $i=1 \ldots c$

$$
\begin{aligned}
j= & 1 \ldots b \\
v= & 1 \ldots v \\
y_{i . v}= & \text { média do bloco } j, \text { no ano } v, \text { (só com os tratamen- } \\
& \quad \text { tos comuns). } \\
y . j v= & \text { média do tratamento } i \text { no ano } v . \\
y^{. . v v}= & \text { média geral do experimento, no ano } v, \text { (só com os } \\
& \text { tratamentos comuns). }
\end{aligned}
$$

A seguir calcula-se a matriz $\mathbf{G}=$ (gss'), que $\bar{e}$ sob Ho, uma estimativa não tendenciosa da matriz de covariân cia comum $\$$.

$$
\begin{aligned}
\mathbf{G}=(c-1)^{-1} \sum_{i} \mathbf{G}_{i}=[(c-1)(b-1)]^{-1} \sum_{j}\left(y i j v^{-y} i . v^{-y} \cdot j v^{+y} \ldots v\right) \mathbf{x} \\
\quad x\left(y_{i j v^{\prime}}+y_{i, v^{\prime}}{ }^{-y} \cdot j v^{\prime}+y v^{\prime}\right)
\end{aligned}
$$


Calculando-se a seguir, $g_{1} ; g_{2}, g_{3}$, onde

$g_{1}=\frac{(c-1) v(v+1)}{2}$

$g_{2}=\frac{\left(2 v^{2}+3 v-1\right)\left(c^{2}-c-1\right)}{6(v+1)(b-1)(c-1)}$

$g_{3}=[|b-1| c]^{\prime} \ln |\mathbf{G}|-\sum_{l}\left[(b-1) \ln \left|\mathbf{G}_{i}\right|\right]$

onde $\mid$, indica o determinante da matriz e $\ln$, o logaritmo neperiano; assim tem-se a distribuição aproximada; $\left(1-g_{2}\right) g_{3} \sim x_{g_{1}}^{2}$, se $\left(1-g_{2}\right) g_{3}<x_{g_{1}}^{2}$, aceita-se a hipótese de igualdade das matrizes de covariâncias para tratamentos.

4.10.2. Teste de Uniformidade da Matriz Comum de Covariâncias.

Para o teste de uniformidade da matriz comum de co variâncias, utilizou-se o critério descrito por DINIZ(1980). Seja Ho $=\psi=\$ *$, onde $\psi^{*}$ é uma matriz uniforme calcularse assim

a) $\gamma=\frac{|\mathbf{G}|}{|\bar{G}|}$ 
onde

$\overline{\mathbf{G}}$, ê uma matriz onde os elementos da diagonal principal isão iguais a média aritmética dos elementos da diagonal principal de G, e os elementos fora da diagonal principal, são também iguais a mêdia aritmética dos elementos de G, que estão fora da diagonal principal.

b) $g_{4}=-(c-1)(b-1) \ln \Upsilon$

c) $g_{5}=\frac{v(v+1)^{2}(2 v-3)}{6(c-1)(b-1)(v-1)\left(v^{2}+v-4\right)}$

d) $g_{6}=\frac{v^{2}+v-4}{2}$

Como $\left(1-g_{5}\right) g_{4} \sim x_{9_{6}}^{2}$, se $\left(1-g_{5}\right) g_{4}<x_{g_{6}}^{2}$, aceita-se a hipôtese de que a matriz de covariância, G é do tipo "uniforme".

E importante salientar, que de acordo com IEMMA (1982), após a aceitação das hipóteses de homogeneidade e unifor midade das matrizes de covariâncias, os valores singulares, $\lambda_{1}$ de multiplicidade de 1 e $\lambda_{2}$ de multiplicidade $(v-1)$, da matriz

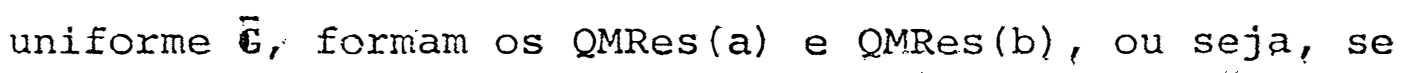
$\operatorname{QMRes}(a)=\left[\begin{array}{llll}1+(v-1) & \rho\end{array}\right] \sigma^{2}$, então $\lambda_{1}+(v-1) \lambda_{2}=\operatorname{QMRes}(a)$ (41025) e $\operatorname{QMRes}(b)=(1-\rho) \sigma^{2}$, então $\lambda_{1}-(v-1) \lambda_{2}=\operatorname{QMRes}(b) \cdot(41026)$ 


\section{RESULTADOS E DISCUSSÃo}

Considerando os dados da tabela 1, aplicando- se o método descrito no capitulo anterior, chegou-se aos seguintes resultados:

5.1. Testes de Homogeneidade e Uniformidade da Matriz de Covariâncias

Segundo DINIZ (1980), e outros, estes testes fazem necessários, por serem eles umas das exigências do modelo ma temático.

Considerando a metodologia descrita em 4.10 têmse as 5 estimativas das matrizes de covariâncias, para os 5 tra- 
tamentos comuns, uma vez que os tratamentos regulares não tem repetições.

De $(410.11)$

$$
\begin{aligned}
& \mathbf{G}_{1}=\left|\begin{array}{ll}
12,9089 & 13,4347 \\
13,4347 & 19,9322
\end{array}\right| \quad \therefore \quad \begin{aligned}
\left|\mathbf{G}_{1}\right| & =76,8116 \\
\ln \left|\mathbf{G}_{1}\right| & =4,3414
\end{aligned} \\
& \mathbf{G}_{2}=\left|\begin{array}{ll}
13,2541 & 18,0046 \\
18,0046 & 49,1399
\end{array}\right| \quad \therefore \quad \begin{aligned}
\left|\mathbf{G}_{2}\right| & =327,1395 \\
\ln \left|\mathbf{G}_{2}\right| & =5,7904
\end{aligned} \\
& \mathbf{G}_{3}=\left|\begin{array}{ll}
25,0137 & 21,9439 \\
21,9439 & 62,6415
\end{array}\right| \quad \therefore \quad \begin{aligned}
\left|\mathbf{G}_{3}\right| & =1.085,3609 \\
\ln \left|\mathbf{G}_{3}\right| & =6,9897
\end{aligned} \\
& \mathbf{G}_{4}=\left|\begin{array}{ll}
21,0569 & -7,0743 \\
-7,0743 & 29,9761
\end{array}\right| \quad \therefore \quad \begin{aligned}
\left|\mathbf{G}_{4}\right| & =581,1580 \\
\ln \left|\mathbf{G}_{4}\right| & =6,3650
\end{aligned} \\
& \mathbf{G}_{5}=\left|\begin{array}{rr}
11,6403 & 8,2234 \\
8,2234 & 36,2699
\end{array}\right| \quad \therefore \quad \begin{aligned}
\left|\mathbf{G}_{5}\right| & =354,5682 \\
\ln \left|\mathbf{G}_{5}\right| & =5,8709
\end{aligned}
\end{aligned}
$$

a estimativa G, para a matriz comum de covariância $\$$, usando $(4101.2)$

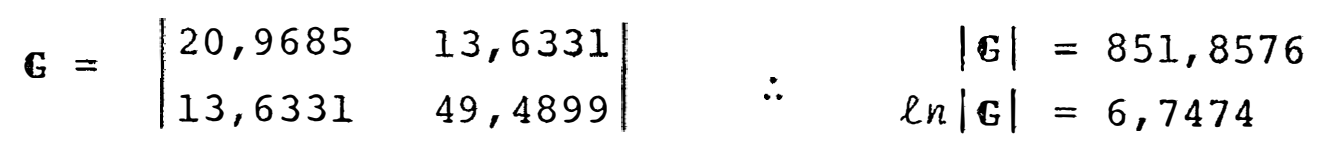


de $(4101.3) ;(4101.4)$ e (4101.5) tem-se

$g_{1}=12$

$g_{2}=0,2144$

$g_{3}=17,5184$

$\therefore\left(1-g_{2}\right) g_{3}=13,76$

$x^{2}\left(5 \%, g_{1}\right)=21,00$

como $13,76<21,00$ aceitamos $\mathrm{H}_{\mathrm{O}}$, as matrizes são homogeneas. Para testar a uniformidade, usando 4102.1 obteve-

se

$\overline{\mathbf{G}}=\left|\begin{array}{ll}35,2292 & 13,6331 \\ 13,6331 & 35,2292\end{array}\right| \quad \therefore \quad|\overline{\mathbf{G}}|=1055,2351$

$\therefore \quad r=\frac{|\mathbf{G}|}{|\overline{\mathbf{G}}|}=\frac{851,8676}{1055,2351}=0,8073 ; \quad \ln |\boldsymbol{\gamma}|=-0,2141$

de $(4102.2) ;(4102.3) ;(4102.4)$ obteve-se

$g_{4}=3,4256 \quad g_{5}=0,0938 \quad g_{6}=1$

$\operatorname{assim}\left(1-g_{5}\right) g_{4}=3,1037$ e $x^{2}(5 \%, 1)=3,84$

aceitando-se assim a uniformidade das matrizes.

Satisfeita as exigências do modelo matemático adotą

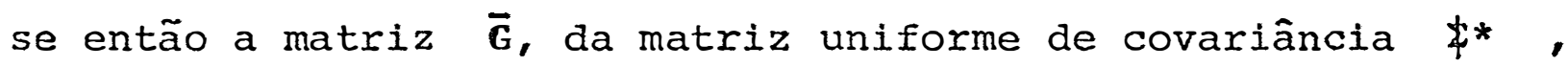
para todos os tratamentos. 
5.2. Equações Normais

Considerando o modelo matemätico $(42.2)$ e 'sendo $\ell=40$ tratamentos principais, onde

$$
\begin{aligned}
& c=5 \text { tratamentos comuns } \\
& z=35 \text { tratamentos regulares. }
\end{aligned}
$$

$b=5$ blocos

$v=2$ tratamentos secundários (cortes)

$K=12$ parcelas por blocos, sendo $K=c+k ; e$

$$
\begin{aligned}
& c=5 \text { para os comuns } \\
& k=7 \text { para os regulares }
\end{aligned}
$$

$n=b K v=120$, total de sub-parcelas.

Tem-se assim o sistema de equações normais

$(43.2)$, cujas soluções são vistas na tabela 2 , obtidas utilizando-se das seguintes equações:
a) para a média, usou-se (44.1)
b) para os tratamentos principais, usou-se (44.11)
c) para os blocos, usou-se (44.15)
d) para os anos, usou-se (44.18)
e) para as interações txa, usou-se nas interações (comuns $x$ anos) a (44.26); para (regulares $x$ anos) a (44.28)
f) para as interações bxa, usou-se (44.23). 
5.3. Quadro de Anālise de Variância

Para obtenção das somas de quadrados usou-se as seguintes equações:

a) Soma de quadrados para blocos ( $452.4 \mathrm{a})$

b) Soma de quadrados tratamentos ajustados ( 452.7 )

c) Soma de quadrados para parcelas (451.6)

d) Soma de quadrados do resíduo (a) (452.10)

e) Soma de quadrados para os anos (452.13)

f) Soma de quadrados da interação txa, usou-se tâ'TA

g) Soma de quadrados da interação bxa, usou-se bâ'BA

h) Soma de quadrados do Total ; Y'Y - $\mathrm{C}_{\mathrm{O}}$

i) Soma de quadrados do resíduo (b), usou-se SQRes (b) = SQTotal - SQP(b) - SQParcelas. 
Obtendo-se assim o quadro de anâlise de variância

\begin{tabular}{l|r|r|r|r}
\hline C.Variação & GL & \multicolumn{1}{|c|}{ S.Quadrados } & Q.Médio & \multicolumn{1}{c}{ F } \\
\hline Blocos & 4 & 2714,713000 & 678,67825 & \\
trat.ajust. & 39 & 28946,869060 & 742,22741 & $15,190 * *$ \\
Res (a) & 16 & 781,786940 & 48,86168 & \\
\hline Parcelas & 59 & $32.443,369000$ & & \\
\hline anos & 1 & 25708,134000 & $25.708,13400$ & $1190,00 * *$ \\
txa & 39 & 5350,785269 & 137,19962 & \\
bxa & 4 & 169,644826 & 42,41121 & \\
Res (b) & 16 & 345,6539050 & 21,60337 & \\
\hline Total & 119 & $64.017,587000$ & & \\
\hline
\end{tabular}

** significativo a $1 \%$

Obs : :

1) Não foram realizados os testes "F", para blocos por não ter interesse prático, e igualmente para as interações ' txa, bxa.

2) Observou-se também, que de acordo com FEDERER (1956), para experimentos envolvendo modelos aumentados, onde os tratamentos regulares não tem repetições, os residuos (a) e (b) podem ser obtidos fazendo-se uma análise somente com os tratamentos comuns. 
3) Segundo IFMMA (1981) , os resíduos (a) e (b) ,podem tambêm ser obtidos dos valores singulares da matriz uniforme $\overline{\mathbf{G}}(4102.5)$ sendo $\lambda_{1}=35,2292$ e $\quad \lambda_{2}=13,6331, \operatorname{assim}, \operatorname{Res}(a)=\lambda_{1}+\lambda_{2}$

$$
\operatorname{Res}(b)=\lambda_{1}-\lambda_{2}
$$

5.4. Comparações Mūitiplas

Para as comparações múltiplas, entre os tratamentos principais, tem-se as seguintes D.M.S., considerando $\alpha=5 \%$

a) para contrastes entre dois tratamentos comuns, usou-se (491.1)

$$
\mathrm{DMS}_{1}=q \sqrt{\frac{48,86168}{10}}=14,5670
$$

b) para contrastes entre um tratamento comum e um regular. usou-se $(491.2)$

$$
\begin{aligned}
\text { DMS }_{2} & =q \sqrt{\frac{1}{2}\left(\frac{1}{2}+\frac{1}{10}+\frac{1}{10}-\frac{1}{50}\right) 48,86168}= \\
& =26,8602
\end{aligned}
$$

c) para dois tratamentos regulares dentro do mesmo bloco, usou se $(491.3)$

$$
\mathrm{DMS}_{3}=\mathrm{q} \sqrt{\frac{48,86168}{2}}=32,5728
$$




\section{Quadro de diferenças mínimas significativas} Trat.

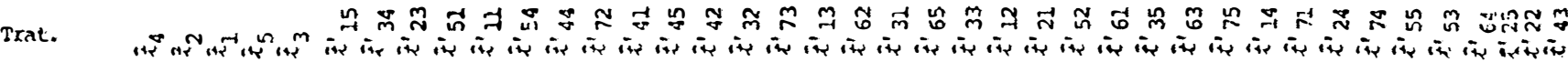
Mïdias

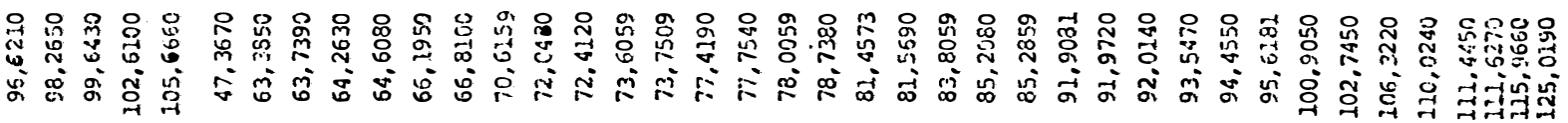

\begin{tabular}{|c|c|}
\hline $\bar{t}_{3}$ & 105,6660 \\
\hline & 102,6100 \\
\hline & 99,6430 \\
\hline & 98,2650 \\
\hline & 96,6210 \\
\hline & 125,0190 * \\
\hline & 115,9660 \\
\hline & $.111,6270$ \\
\hline & 111,4450 \\
\hline & 110,0240 \\
\hline & 106,3220 \\
\hline & 102,7450 \\
\hline & 100,9050 \\
\hline & 95,6181 \\
\hline & 94,4550 \\
\hline & 93,5470 \\
\hline & 92,0140 \\
\hline & 91,9720 \\
\hline & 91,9081 \\
\hline & 85,2859 \\
\hline & 85,2080 \\
\hline & 83,8059 \\
\hline & 81,5690 \\
\hline & 81,4573 \\
\hline & 78,7380 \\
\hline & 78,0059 \\
\hline & 77,7540 \\
\hline & 77,4190 \\
\hline & 73,7509 \\
\hline & 73,6059 \\
\hline & 72,4120 \\
\hline & 72,0480 \\
\hline & 70,6159 \\
\hline & 66,8100 \\
\hline & 66,1950 \\
\hline & 64,6080 \\
\hline & $64,26,30$ \\
\hline & 63,7390 \\
\hline & 63,3850 \\
\hline & 47,3670 \\
\hline
\end{tabular}

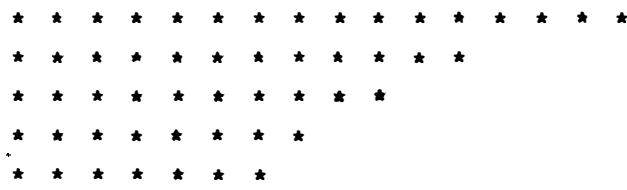




\section{CONCLUSŌES}

1) A análise de delineamentos aumentados (Blocos de FEDERER) no esquema de parcelas subdivididas no tempo é perfeitamen te viāvel.

2) Nesse contexto, deve ser feita após o teste de homogeneidade e uniformidade de variâncias ter-se mostrado não significativo.

3) Quando os tratamentos regulares aparecem somente uma vez em todo o experimento os resíduos podem ser obtidos da seguinte forma:

3.1. Através dos valores singulares da matriz uniforme

3.2. Através de uma análise, feita somente com os tratamen tos comuns

3.3. Através do modelo adotado. 


\section{REFERENCIAS BIBLIOGRÁFICAS}

AFONJA, A., 1968. Analysis of a Group of Balanced Block Experiments Hawing Erros Variance and Some Treatments in Common. Biometrics, $24: 389-411$.

ANDERSON, R.L. e T.A. BANCROFT, 1952. Statistical theory in research. McGraw-Hill Book Co., Inc., Nova York . 399 pp.

BOX, G.E.P., 1949. A General Distribution Theory for a Class of Likelihood Criteria. Biometrika, Londres, 36: $317-346$.

BOX, G.E.P., 1950. Problems in the Analysis of Growth and Wear Curves. Biometrics, Carolina do Norte, 6: $362-389$. 
CHAKRABART, M.C., 1962. Mathematics of Design and Analysis of Experiments. 18 ed. Asia Publishing House , Londres, $120 \mathrm{pp}$.

COCHRAN, W.G. e G.M. COX, 1976. Diseños Experimentales. 38 ed. Trillas, México, 661 pp.

CORSTEN, L.C.A., 1962. Balanced Block Desing with two Dif ferent Number of Replicates. Biometrics, 18: 499-519.

DANFORD, M.B.; H.M. HUGHES A R.C. MCNEE, 1960. On the Analysis of Repeated-Measurements Experiments. Biometrics, Raleigh, 16: 547-565.

DINIZ, U.D., 1980. Análise de Experimentos com Parcelas Medidas Sucessivamente no Tempo. ESALQ/USP, Piracicaba, 104 pp. (Tese de Doutoramento).

FEDERER, W.T., 1955. Experimental Design. 18 ed., MCMil lan Co., Nova York, 544 pp.

FEDERER, W.T., 1956. Augmented (or Hoonuiaku) designs. Hawaiian Planter's Record 55: 191-208. 
FEDERER, W.T., 196la. Augmented designs With one way eli mination of heterogeneith. Biometrics 17: 447-473.

FEDERER, W.T., 196lb. Augmented designs with two three and higher was elimination of heterogeneity (abstract). Biometrics 17: 166 .

FEDERER, W.T.; RAGHAVARAO, D., 1975. On augmented designs. Biometrics 31: 29-35.

FEDERER, W.T.; NAIR, R.C.; RAGHAVARAO, D., 1975. Some augmented row - column designs. Biometrics 31: 361373.

GEISSER, S., 1963. Multivariate Analysis of Variance for a Special Covariance Case. Journal of the American Statistical Association, Washington, 58: 660-669.

GILI, L.C., 1978. Design and Analysis of Experiments in the Animal and Medical Sciences. The Iowa State University Press. 19 ed. Ames, 3 vol. 882 pp. 
IEMMA, A.F., 1981. Anālise de experimentos em parcelas subdivididas com tratamentos principais dispostos em blocos incompletos balanceados. ESALQ/USP, Piracicaba, $145 \mathrm{pp}$. (Tese de doutoramento).

IEMMA, A.F., 1982. Valores singulares de matrizes Uniformes, Ciência e Natura (4):21-26.

LEAL, M.L.S., 1979. Análise de Dados Experimentais com Medidas Repetidas. Universidade Federal de Brasilia, Brasília, 99 pp. (Dissertação de Mestrado).

LEONARD, W.H. e A.G. CLARK, 1939. Field Plot Technigue. Burgess. Minneapolis, 288 pp.

MARTINEZ, G.A., 1972. Diseños y análisis de experimentos con cana-de-azúcar. Colégio de Post-Graduados, Escuela Nacional de Agricultura, Chapingo, México.

NOGUEIRA, M.C.S., 1976. Anālise Conjunta para Experimentos em Blocos casualizados completos aumentados , ESALQ/USP, Piracicaba, SP, (Dissertação de Mestrado) .

PARKER, E.R., 1942. Adjustment of Yields in an Experiment with Orange Trees. Proc. Amer. Soc. Hort. Sci., Maryland, 42: 23-33. 
PATTERSON, H.D. e B.I. LOWE, 1970. The Errors of LongTerm Experiments. J.Agric. Sci., Cambridge, 74: 5360 .

PAVATE, M.V., 1961. Combined Analysis of Balanced Incomplet Block Designs with Some Common Treatments . Biometrics, 17: 111-119.

PIMENTEL GOMES, F.; GUIMARÃEs, R.F., 1958. Join Analysis of experiments in complete randomized blocks with some common treatments. Biometrics 14: 521-526.

PIMENTEL GOMES, F., 1967. The solution of normal equations of experimental design models. Ciência e Cultura $19: 567-573$.

PIMENTEL GOMES, F., 1968. The solution of normal equations of experiments in incomplete blocks. Apost. mimeografada, ESALQ/USP.

PIMENTEL GOMES, F., 1970. An extension of the method of joint analysis of experiments in complete randomised blocks. Biometrics 26: 332-336.

PIMENTEL GOMES, F., 1976. Curso de Estatistica. Experi-. mental, 6o edição, Livraria Nobel, São Paulo. 
SNEDECOR, G.W., 1956. Statistical Methods. 59 ed. The Iowa State College Press, Ames. Iowa. 534 pp.

SNEDECOR, G.W. e W.G. COCHRAN, 1967. Statistical Methods. 6! edição. The Iowa State University Press,Ames, Iowa. $593 \mathrm{pp}$.

STEEL, R.G.D., 1955. An Analysis of Perennial Crop Data. Biometrics, Carolina do Norte, 11: 201-212.

STEEL, R.G.D. e J.H. TORRIE, 1960. Principles and Procedures of Statistics. MCGraw-Hill Book, Nova York, Toronto e Londres, $481 \mathrm{pp}$.

WILKS, S.S., 1932. Certain Generalizations in the Analysis of Variance. Biometrika, Londres, 24:471-494, 
8. APENDICE 
Tabela 1 - Dados adaptados de um experimento para melhoria de variedades de cana-de-açúcar, realizado em delineamentos de Blocos casualizados completos aumentados (Blocos de FEDERER), com parcelas subdivididas no tempo.

18 BLCCO

\begin{tabular}{|c|c|c|c|c|}
\hline \multicolumn{2}{|c|}{ Varlexdaces } & \multirow{2}{*}{$\begin{array}{l}\text { Prod. Agrlc: } \\
18 \text { corte } \\
112,75\end{array}$} & $\begin{array}{l}\text { Prod. Agric. } \\
28 \text { corte }\end{array}$ & \multirow{2}{*}{$\begin{array}{l}\text { Erod. Thtai } \\
\text { Agricola } \\
200,27\end{array}$} \\
\hline $\mathrm{CB}$ & $41-76$ & & 87,52 & \\
\hline $\mathrm{CB}$ & $36-24$ & 115,43 & 91,03 & 206,51 \\
\hline $\mathrm{CH}$ & $40-13$ & 121,97 & 78,61 & 200,58 \\
\hline $\mathrm{CB}$ & $40-69$ & 106,80 & 82,76 & 189,56 \\
\hline $\mathrm{CB}$ & $41-14$ & 117,51 & 90,50 & 208,01 \\
\hline $\mathrm{CB}$ & $6 I-2$ & 75,86 & 53,26 & 129,12 \\
\hline $\mathrm{CB}$ & $53-98$ & 82,41 & 87,91 & 170,32 \\
\hline $\mathrm{CB}$ & $58-i 1$ & 102,34 & 55,04 & 157,38 \\
\hline $\mathrm{CB}$ & $47-31$ & 92,14 & 53,86 & 144,00 \\
\hline $\mathrm{CH}$ & $61-4$ & 77,94 & 50,49 & 128,43 \\
\hline IAC & $51-205$ & 205,31 & 78,41 & 183,72 \\
\hline Co & 678 & 100,85 & 90,29 & 191,14 \\
\hline
\end{tabular}

38 BLOCO

\begin{tabular}{|c|c|c|c|c|}
\hline \multicolumn{2}{|c|}{ Variedzdcs } & \multirow{2}{*}{$\begin{array}{l}\text { Prod. Agric. } \\
18 \text { corte }\end{array}$} & \multirow{2}{*}{$\begin{array}{l}\text { Prod. Agric. } \\
27 \text { corte } \\
79,20\end{array}$} & \multirow{2}{*}{$\begin{array}{l}\begin{array}{l}\text { Prod. Total } \\
\text { Ajricola }\end{array} \\
179,75\end{array}$} \\
\hline $\mathrm{CB}$ & $41-76$ & & & \\
\hline$C 8$ & $36-24$ & 104,42 & 78,80 & 183,22 \\
\hline $\mathrm{CB}$ & $40-13$ & 118,10 & 76,43 & 194,53 \\
\hline $\mathrm{CB}$ & $40-69$ & 112,45 & 80,20 & 192,65 \\
\hline $\mathrm{CB}$ & $41-14$ & 210,37 & 93,65 & 204,02 \\
\hline $\mathbb{C B}$ & $55-49$ & 82,11 & 63,15 & 145,25 \\
\hline $\mathrm{CB}$ & $55-65$ & 68,13 & 49,10 & 117,23 \\
\hline $\mathrm{CB}$ & $55-72$ & 96,09 & 56,80 & 152,89 \\
\hline IAC & $52-172$ & 124,95 & 114,84 & 239,79 \\
\hline IAC & $52-179$ & 125,25 & 84,55 & 209,80 \\
\hline IAC & $55-26$ & 82,70 & 91,08 & 173,78 \\
\hline $\mathrm{CB}$ & $61-14$ & 84,19 & 60,40 & 144,59 \\
\hline \multicolumn{5}{|c|}{ - } \\
\hline \multicolumn{2}{|c|}{ Varledaces } & $\begin{array}{l}\text { Prod. Agric. } \\
18 \text { ocrie }\end{array}$ & $\begin{array}{l}\text { Prod. igric. } \\
28 \text { corte. }\end{array}$ & $\begin{array}{l}\text { Prod. Total } \\
\text { Agricola }\end{array}$ \\
\hline $\mathrm{CC}$ & $41-76$ & 105,31 & 86,50 & 191,81 \\
\hline $\mathrm{CB}$ & $36-24$ & 104,72 & $7 i, 10$ & 175,82 \\
\hline $\mathrm{CB}$ & $40-13$ & 128,22 & 96,60 & 224,82 \\
\hline $\mathrm{Cs}$ & $40-69$ & 105,31 & 84,35 & 189,66 \\
\hline $\mathrm{CB}$ & $41-14$ & 114,83 & 82,75 & 197,58 \\
\hline $\mathrm{CB}$ & $55-27$ & 58,31 & 31,28 & 89,59 \\
\hline LANE: & $55-34$ & 129,41 & 88,70 & 218,11 \\
\hline IALNE & $55-45$ & 121,38 & 57,42 & 178,80 \\
\hline$C B$ & $60-9$ & 73.18 & 66,50 & 139,68 \\
\hline $\operatorname{Cr}$ & $61-99$ & 124,95 & 82,55 & 207,50 \\
\hline $\mathrm{CB}$ & $61-76$ & 85,87 & 70,90 & 157,77 \\
\hline $\mathrm{CB}$ & $61-81$ & 119,60 & 62,35 & $i 81,35$ \\
\hline
\end{tabular}

29 BLOCO

\begin{tabular}{|c|c|c|c|c|}
\hline \multicolumn{2}{|c|}{ Verrleciades } & \multirow{2}{*}{$\begin{array}{l}\text { Prod.Agric. } \\
18 \text { corte } \\
112,46\end{array}$} & \multirow{2}{*}{$\begin{array}{l}\text { Prot. Agatc. } \\
28 \text { corte } \\
83,95\end{array}$} & $\begin{array}{l}\text { Prod. Total } \\
\text { Agricula }\end{array}$ \\
\hline $\mathrm{CP}$ & $41-76$ & & & 196,41 \\
\hline $\mathrm{CB}$ & $36-24$ & 117,81 & 82,96 & 200,77 \\
\hline $\mathrm{CB}$ & $40-13$ & 119,00 & 86,92 & 205,92 \\
\hline $\mathrm{CB}$ & $40-69$ & 114,83 & 73,66 & 183,49 \\
\hline $\mathrm{CB}$ & $41-14$ & 117,21 & 89,50 & 206,71 \\
\hline $\mathrm{CB}$ & $40-11$ & 100,85 & 65,34 & 166,19 \\
\hline IAC & $50-134$ & 145,77 & 84,74 & 230,51 \\
\hline IAC & $48-65$ & 91,04 & 55,04 & 146,08 \\
\hline $\mathrm{CP}$ & $53-18$ & 97,28 & 48,51 & 145,79 \\
\hline Co & 453 & 97,28 & 71,87 & 169,15 \\
\hline$R R$ & 980 & 83,90 & 70,69 & 154,59 \\
\hline$C B$ & $55-21$ & 82,41 & 57,40 & 139,81 \\
\hline
\end{tabular}

$49 \quad$ BIOCO

\begin{tabular}{|c|c|c|c|c|}
\hline \multicolumn{2}{|c|}{ Varledades } & \multirow{2}{*}{$\begin{array}{l}\text { Prod. Agcic. } \\
18 \text { vorte } \\
126,44\end{array}$} & \multirow{2}{*}{$\begin{array}{l}\text { Prod. Agric. } \\
28 \text { onrte } \\
101,75\end{array}$} & \multirow{2}{*}{$\begin{array}{l}\text { Pmd. Total } \\
\text { Agríoola } \\
228,19\end{array}$} \\
\hline $\mathrm{CB}$ & $41-76$ & & & \\
\hline $\mathrm{CB}$ & $36-24$ & $12 \vdots, 68$ & 94,65 & 216,33 \\
\hline$C B$ & $40-13$ & 135,36 & 95,45 & 230,81 \\
\hline $\mathrm{CB}$ & $40-69$ & 125,25 & 79,60 & 204,85 \\
\hline $\mathrm{CB}$ & $41-14$ & 120,48 & 89,30 & 209,78 \\
\hline$A N$ & $56-62$ & 118,70 & 87,12 & 205,82 \\
\hline $\mathrm{CP}$ & $48-117$ & 117,72 & 101,00 & 218,72 \\
\hline$C P$ & $50-28$ & 85,68 & 58,00 & 143,68 \\
\hline $\mathrm{CB}$. & $52-46$ & 91,33 & 59,20 & 150,53 \\
\hline $\mathrm{CB}$ & $5 \varepsilon-30$ & 77,05 & 72,25 & 149,30 \\
\hline $\mathrm{CB}$ & $58-179$ & 136,25 & 103,55 & 239,80 \\
\hline$C B$ & $61-150$ & 136,85 & 85,55 & 222,40 \\
\hline
\end{tabular}


.122

Tabela 2 - Valores abservados dos parâmetros e suas estimativas.

\begin{tabular}{|c|c|c|c|c|c|c|c|}
\hline Parâmetros & & Valor & or Observado & Valor de 0 & 8 & $\begin{array}{c}\text { Solucão das } \\
\text { Eq. Yort:uds }\end{array}$ & Afuste \\
\hline G & & 109 & 970,27 & - & $\hat{m}$ & 91,4189 & - \\
\hline$T_{2}=\mathrm{CB}$ & $41-76$ & & 996,43 & 82,2408 & $\bar{t}_{1}$ & 8,22408 & 99,6430 \\
\hline$T_{2}=\mathrm{CB} \quad 3$ & $36-24$ & & 982,65 & 68,4608 & $\hat{t}_{2}$ & 6,84608 & 98,2650 \\
\hline$T_{3}=\mathrm{CB} \quad 4$ & $40-13$ & 10 & 056,66 & 142,4708 & $\hat{t}_{3}$ & 14,24708 & 105,6660 \\
\hline$E_{4}=\mathrm{CB} \quad 4 \mathrm{C}$ & $40-69$ & & 965,21 & 51,0208 & $\hat{t}_{4}$ & 5,10208 & 96,5210 \\
\hline$\tau_{5}=\mathrm{CB}$ & $41-14$ & 10 & 026,10 & 111,9108 & $\hat{f}_{5}$ & 11,19108 & 102,6100 \\
\hline$T_{11}^{\prime}=\mathrm{CB} \quad 6$ & $61-2$ & & 129,12 & $-46,6333$ & $\hat{t}_{i 1}$ & $-26,8109$ & 64,6080 \\
\hline$E_{21}^{\prime}=\mathrm{CB} \quad 5$ & $53-98$ & & 170,32 & $-5,4333$ & $\hat{t}_{21}^{\prime}$ & $-6,2109$ & 85,2080 \\
\hline$T_{31}^{\prime}=\mathrm{CB} \quad 5$ & $58-11$ & & 257,38 & $-18,3733$ & $\bar{t}_{31}^{\prime}$ & $-12,6809$ & 78,7380 \\
\hline$T_{41}^{\prime}=\mathrm{CB}$ & $47-31$ & & 144,00 & $-31,7533$ & $\hat{t}_{41}^{\prime}$ & $-19,3709$ & 72,0480 \\
\hline$T_{51}^{\prime}=\mathrm{CB} \quad 6$ & $61-4$ & & 128,43 & $-47,3233$ & $\hat{t}_{51}^{\prime}$ & $-27,1559$ & 64,2630 \\
\hline$T_{61}^{\prime}=\operatorname{IAC} 5$ & $51-205$ & & 183,72 & 7,9667 & $\hat{t}_{61}^{\prime}$ & 0,4892 & 91,9081 \\
\hline$T_{71}^{\prime}=\mathrm{CO} \quad 6$ & 678 & & 191,14 & 15,3867 & $\tilde{t}_{71}^{\prime}$ & 4,1992 & 95,6181 \\
\hline$T_{12}^{\prime}=\mathrm{CB} \quad 4$ & $40-11$ & & 166,19 & $-13,0117$ & $\hat{t}_{12}$ & $-7,6130$ & 83,8059 \\
\hline$T_{\angle 2}^{\prime}=\operatorname{IAC} 5$ & $50-134$ & & 230,52 & 51,3083 & $\hat{t}_{22}^{\prime}$ & 24,5471 & 215,9660 \\
\hline$T_{32}^{\prime}=\operatorname{IAC} \quad 4$ & $48-65$ & & 146,08 & $-33,1217$ & $\hat{t}_{32}^{\prime}$ & $-17,6680$ & 73,7509 \\
\hline$T_{42}^{\prime}=C P \quad 5$ & $53-18$ & & 145,79 & $-33,4117$ & $\hat{t}_{42}^{\prime}$ & $-17,8130$ & 73,6059 \\
\hline$T_{52}^{\prime}=\mathrm{Co} \quad 4$ & 453 & & 169,15 & $-10,0517$ & $\bar{t}_{52}^{\prime}$ & $-6,1330$ & 85,2859 \\
\hline$T_{62}^{\prime}=\mathrm{PR} \quad 9$ & 980 & & 154,59 & $-24,6117$ & $\hat{t}_{62}^{\prime}$ & $-13,4130$ & 78,0059 \\
\hline$T_{72}^{\prime}=\mathrm{CB} \quad 5$ & $55-21$ & & 139,81 & $-39,3917$ & $\hat{t}_{72}$ & $-20,8030$ & 70,6159 \\
\hline$T_{13}^{2}=\mathrm{CB} \quad 5$ & $55-49$ & & 145,26 & $-32,8658$ & $\hat{t}_{13}$ & $-1.3,6649$ & 77,7540 \\
\hline$t_{23^{\prime}}^{\prime}=\mathrm{CB} \quad \mathrm{S}$ & $55-65$ & & 117,23 & $-60,8958$ & $\hat{t}_{23}^{\prime}$ & $-27,6799$ & 63,7390 \\
\hline$T_{33}^{\prime}=\mathrm{CB} \quad 5$ & $55-72$ & & 152,89 & $-25,2358$ & $\hat{t}_{43}^{\prime}$ & $-9,8499$ & 81,5690 \\
\hline$r_{43}^{\prime}=\operatorname{IAC} 5$ & $52-172$ & & 239,79 & 61,6642 & $\hat{t}_{43}^{\prime}$ & 33,6001 & 125,0190 \\
\hline$T_{53}^{\prime}=$ IAC 5 & $52-179$ & & 209,80 & 31,6742 & $\hat{t}_{53}^{\prime}$ & 18,6051 & 110,0240 \\
\hline$T_{63}^{\prime}=\operatorname{IAC} 5$ & $55-26$ & & 173,78 & $-4,3458$ & $\hat{t}_{63}^{\prime}$ & 0,5951 & 92,0140 \\
\hline$T^{\prime} 3^{\prime}=\mathrm{CB} \quad 6$ & $61-14$ & & 144,59 & $-33,5358$ & $\hat{t}_{2 \cdot 3}^{\prime}$ & $-13,9999$ & 77,4190 \\
\hline$T_{14}^{\prime}=\mathrm{NA} \quad 5$ & $56-62$ & & 205,82 & 4,1358 & $\hat{t}_{14}$ & 3,0351 & 94,4550 \\
\hline$\tau_{24}^{\prime}=\mathrm{Cl} \quad 4$ & $48-117$ & & 218,72 & 17,0358 & $\hat{t}_{24}^{\prime}$ & 9,4861 & 100,9050 \\
\hline$r_{34}^{\prime}=\mathrm{CP} \quad 5$ & $50-28$ & & 143,68 & $-58,0042$ & $\hat{t}_{34}^{\prime}$ & $-28,0.339$ & 63,3850 \\
\hline$T_{44}^{\prime}=\mathrm{CB} \quad 5$ & $52-46$ & & 150,53 & $-51,1542$ & $\hat{t}_{44}^{\prime}$ & $-24,6089$ & 66,8100 \\
\hline$T_{54}^{\prime}=C B \quad 5$ & $56-30$ & & 149,30 & $-52,3842$ & $\hat{t}_{54}^{\prime}$ & $-25,2239$ & 66,1950 \\
\hline$T_{64}^{\prime}=\mathrm{CB} \quad 5$ & $58-179$ & & 239,80 & 38,1158 & $\hat{t}_{64}^{\prime}$ & 20,0261 & 111,4450 \\
\hline$T_{74}^{\prime}=\mathrm{CB} \quad 6$ & $61-150$ & & 222,40 & 20,7158 & $\hat{t}_{74}^{\prime}$ & 11,3261 & 102,7450 \\
\hline$T_{15}^{\prime}=C B \quad 5$ & $55-27$ & & 87,59 & $-89,8342$ & $\hat{t}_{15}^{\prime}$ & $-44,0519$ & 47,3670 \\
\hline$T_{25}^{\prime}=$ IANE & $85-34$ & & 218,11 & 38,6858 & $\hat{t}_{25}^{\prime}$ & 20,2081 & 111,6270 \\
\hline$T_{35}^{\prime}=$ IANE & $55-45$ & & 278,80 & $-0,6242$ & $\hat{t}_{35}^{\prime}$ & 0,5531 & 91,9720 \\
\hline$T_{45}^{\prime}=\mathrm{CB}$ & $60-9$ & & 139,53 & $-39,7442$ & $\hat{t}_{45}^{\prime}$ & $-19,0069$ & 72,4120 \\
\hline$r_{55}^{\prime}=\mathrm{CB} \quad 6$ & $61-99$ & & 207,50 & 28,0758 & $\hat{x}_{5 s}^{\prime}$ & 24,9031 & 106,3220 \\
\hline$T_{65}^{\prime}=\mathrm{CB} \quad 6$ & $61-76$ & & 157.77 & $-21,6542$ & $\bar{t}_{6,5}^{\prime}$ & $-3,3619$ & 81,4573 \\
\hline $\mathrm{r}_{75}^{\prime}=\mathrm{CB}$ & $61-81$ & & 181,95 & 2,5258 & $\hat{t}_{75}^{\prime}$ & 2,1281 & $9.3,5470$ \\
\hline
\end{tabular}


Tabela 2 - (continuação).

\begin{tabular}{|c|c|c|c|c|c|}
\hline Parämetros & Valor Observado & Valor de 0 & 3 & $\begin{array}{l}\text { Soluçáo das } \\
\text { Sq Morresta }\end{array}$ & Ajuste \\
\hline${ }_{1}^{8}$ & 2109,04 & - & $\bar{b}_{1}$ & $-0,0480$ & \\
\hline$B_{2}$ & 2150,42 & - & $\hat{b}_{2}$ & $-0,7110$ & \\
\hline $8_{3}$ & 2137,51 & - & $\hat{b}_{3}$ & $-5,1240$ & \\
\hline $8_{4}$ & 2420,21 & - & $\bar{b}_{4}$ & 8,4550 & \\
\hline$B_{5}$ & 2153,09 & - & $\hat{b}_{5}$ & $-2,5720$ & \\
\hline$A_{1}$ & 6363,34 & - & $\bar{a}_{1}$ & 14,6368 & \\
\hline$A_{2}$ & 4606,93 & - & $\hat{a}_{2}$ & $-14,6368$ & \\
\hline$T A_{11}$ & 557,51 & - & $t_{a 1}$ & $-2,7778$ & \\
\hline$T A_{21}$ & 564,06 & - & $\hat{\mathrm{ia}}_{21}$ & $-0,0898$ & \\
\hline$T A_{31}$ & 622,65 & - & $\tilde{t a}_{31}$ & 4,2272 & \\
\hline$T A_{41}$ & 564,64 & - & $\tilde{t a}_{41}$ & 1,7702 & \\
\hline$T A_{51}$. & 580,40 & - & $\hat{t a}_{51}$ & $-1,1668$ & \\
\hline $\mathrm{TA}_{12}$ & 438,92 & - & $\dot{t a}_{12}$ & 2,7778 & \\
\hline$T A_{22}$ & 418,59 & - & $\hat{t a}_{22}$ & 0,0898 & \\
\hline$T A_{32}$ & 434,01 & - & $t_{32}$ & $-4,2272$ & \\
\hline$T A_{42}$ & 400,57 & - & $\tilde{t a}_{42}$ & $-1,7702$ & \\
\hline$T A_{52}$ & 445,70 & - & $t a_{52}$ & 1,1668 & \\
\hline$T: A_{111}$ & 75,86 & - & $t^{t} a 11$ & $-2,7064$ & \\
\hline$T^{\prime} A_{211}$ & 82,41 & - & $t 211$ & $-16,7564$ & \\
\hline$T^{\prime} A_{311}$ & 102,34 & - & $t^{t} a_{311}$ & 9,6436 & \\
\hline$T^{\prime} A_{411}$ & 90,14 & 2 & ${ }^{5} a_{411}$ & 4,1336 & \\
\hline$T^{\prime} A_{511}$ & 77,94 & - & $t^{3} a_{511}$ & $-0,2814$ & \\
\hline$T^{\prime} A_{611}$ & 105,31 & - & $t^{5} a_{611}$ & $-0,5564$ & \\
\hline$T^{\prime} A_{711}$ & 100,85 & - & $t^{f} a 11$ & $-8,7264$ & \\
\hline$T^{\prime} A_{121}$ & 100,85 & - & $t^{t a} a 21$ & 1,7156 & \\
\hline$T^{\prime} A_{221}$ & 145,77 & - & $t^{5} a_{221}$ & 14,4756 & \\
\hline$T^{\prime} A_{321}$ & 91,04 & - & $i^{5} a_{321}$ & 1,9606 & \\
\hline$T^{\prime} A_{421}$ & 97,28 & - & $t^{2} a_{421}$ & 8,3456 & \\
\hline$T^{\prime} A_{521}$ & 97,28 & - & $t^{\prime} a_{521}$ & $-3,3344$ & \\
\hline$T \cdot A_{621}$ & 83,90 & - & $t a_{621}$ & $-9,4344$ & \\
\hline$T^{\prime} A_{721}$ & 82,41 & - & $\begin{array}{l}t a \\
t a\end{array}$ & $-3,5344$ & \\
\hline$T^{\prime} A_{131}$ & 82,11 & - & $2 a 131$ & $-3,8884$ & \\
\hline$T^{\prime} A_{231}$ & 68,13 & - & $\operatorname{la}_{231}$ & $-3,8534$ & \\
\hline$T^{\prime} A_{331}$ & 96,09 & - & $t^{t} a 31$ & 6,2766 & \\
\hline$T^{\prime} A_{431}$ & 124,95 & - & $a_{431}$ & $-8,3134$ & \\
\hline$r \cdot A_{531}$ & 125,25 & - & $t^{\prime} a_{531}$ & 6,9816 & \\
\hline$T^{\prime} A_{631}$ & 82,7 & - & $t^{2} a_{631}$ & $-17,5584$ & \\
\hline$T^{\prime} A_{731}$ & 84,19 & - & $t^{\prime} a 731$ & $-1,4734$ & \\
\hline$T \cdot A_{141}$ & 118,70 & - & $t^{t} a_{141}$ & $-0,6633$ & \\
\hline$T^{\prime} A_{241}$ & 117,72 & - & $\operatorname{ta}_{241}$ & $-8,0933$ & \\
\hline
\end{tabular}


Tabela 2 - (continuação).

\begin{tabular}{|c|c|c|c|c|}
\hline Valor observado & Valox da 2 & 8 & $\begin{array}{l}\text { Solução das } \\
\text { Eq. Nornats }\end{array}$ & Ajuste \\
\hline 85,68 & - & $t^{t} a_{341}$ & -2.6133 & \\
\hline 91,33 & - & $t^{t} a_{441}$ & $-0,3883$ & \\
\hline 77,05 & - & $t^{2}\left(x_{541}\right.$ & $-14,0533$ & \\
\hline 136,25 & - & $t^{t} a_{642}$ & $-0,1033$ & \\
\hline 136,85 & - & $t^{\prime} a_{741}$ & 9,1967 & \\
\hline 58,31 & - & $t^{\prime} a_{151}$ & 0,1987 & \\
\hline 129,41 & - & $t^{t} a_{251}$ & 7,0387 & \\
\hline 121,38 & - & $t^{5} a_{351}$ & 18,6637 & \\
\hline 73,18 & - & $t^{0} a_{451}$ & $-9,9763$ & \\
\hline 124,95 & - & $t \hat{t} a_{551}$ & 7,8837 & \\
\hline 86,87 & - & $t^{i} a_{651}$ & $-5,3313$ & \\
\hline 119,60 & - & $t^{5} a_{751}$ & 15,3037 & \\
\hline 53,26 & - & $\hat{t}^{\hat{a}}{ }_{112}$ & 2,7064 & \\
\hline 87,91 & - & $\hat{t a}_{212}$ & 16,7564 & \\
\hline 55,04 & - & $t^{\hat{2}} \hat{a}_{312}$ & $-9,6436$ & \\
\hline 53,86 & - & $t^{t} \hat{n}_{412}$ & $-4,1336$ & \\
\hline 50,49 & - & $t \hat{t}_{512}$ & 0,2814 & \\
\hline 78,41 & - & $\hat{t}^{i} a_{612}$ & 0,5564 & \\
\hline 90,29 & - & $t \hat{i a} 712$ & 8,7264 & \\
\hline 65,34 & - & $t^{\hat{i}} a 22$ & $-1,7156$ & \\
\hline 84,74 & - & $t^{2} a_{22}$ & $-14,4756$ & \\
\hline 55,04 & - & $t a 322$ & $\begin{array}{l}-1,9606 \\
-8,3456\end{array}$ & \\
\hline 48,51 & - & $x a_{422}$ & $-8,3456$ & \\
\hline 71,87 & - & $t u_{522}$ & 3,3334 & \\
\hline 70,69 & - & $t_{622}$ & 9,4344 & \\
\hline 57,40 & - & ta 722 & 3,5344 & \\
\hline 63,15 & - & $t a_{132}$ & 3,8884 & \\
\hline 49,10 & - & $t a 232$ & 3,8534 & - \\
\hline 56,80 & - & $t a 332$ & $-6,2766$ & \\
\hline 114,84 & - & $t^{\prime} a_{432}$ & 8,3134 & \\
\hline 84,55 & - & $t^{2} a_{532}$ & $-6,9816$ & \\
\hline 91.08 & - & $\hat{t a} a_{532}$ & 17,5584 & \\
\hline 60,40 & - & $t a 732$ & 1,4734 & \\
\hline 87,12 & - & $t^{3} a 142$ & 0,6633 & \\
\hline 101,00 & - & $t a 242$ & 8,0933 & \\
\hline 58,00 & - & $t^{2} x_{342}$ & 2,6133 & \\
\hline 59.20 & - & $t a_{442}$ & 0,3883 & \\
\hline 72,25 & - & $t a_{542}$ & 14,0533 & \\
\hline 103,55 & - & $t^{t} a_{642}$ & 0,1033 & \\
\hline 85,55 & - & $t^{5}+42$ & $-9,1967$ & \\
\hline 31,28 & - & $t a_{152}$ & $-0,1987$ & \\
\hline 88,70 & - & $\hat{t a n} 252$ & $-7,0387$ & \\
\hline 57,42 & - & tia 352 & $-18,6637$ & \\
\hline
\end{tabular}


Tabela 2 - (continuação).

\begin{tabular}{|c|c|c|c|c|c|c|}
\hline Parämetros & Valc & Lor Observado & Valor de $Q$ & $\hat{\theta}$ & $\begin{array}{l}\text { Soluçäo das } \\
\text { Br. ITorenta }\end{array}$ & Ajuste \\
\hline$T^{\prime} A_{452}$ & & 66,50 & - & $t^{t} a_{452}$ & 9,9763 & \\
\hline$T^{\prime} A_{552}$ & & 82,55 & - & $\hat{t} a_{552}$ & $-7,8837$ & \\
\hline$T^{\prime} A_{652}$ & & 70,90 & - & $\hat{t} a_{652}$ & 5,3313 & \\
\hline$T^{\prime} A_{752}$ & & 62,35 & - & $t^{5} a_{752}$ & $-15,3037$ & \\
\hline$B_{11}$ & & 209,31 & - & $\hat{b a}_{11}$ & $-0,6304$ & \\
\hline $\mathrm{BA}_{21}$ & & 279,84 & - & $\hat{b a}_{21}$ & 1,4026 & \\
\hline$B R_{31}$ & & 209,31 & - & $\overline{b a}_{31}$ & $-1,2684$ & \\
\hline$B A_{41}$ & & 392,79 & - & $\hat{b a}_{14}$ & 1,8166 & \\
\hline${ }^{8 A_{51}}$ & & 272,09 & - & $b a_{51}$ & $-1,3204$ & $\cdot$ \\
\hline${ }^{B A}{ }_{12}$ & & 899,73 & - & $b a_{12}$ & 0,6304 & \\
\hline${ }^{B A_{22}}$ & & 870,58 & - & $\hat{b a}_{22}$ & $-1,4026$ & \\
\hline $\mathrm{BA}_{32}$ & & 928,20 & - & $a_{32}$ & 1,2684 & \\
\hline$B A_{42}$ & & 027,42 & - & $b a_{42}$ & $-1,8166$ & \\
\hline${ }^{B A_{52}}$ & & 881,00 & - & $b a_{52}$ & 1,3204 & \\
\hline
\end{tabular}

